\title{
Tuning the Regioselective Staudinger Reaction for the Facile Synthesis of Kanamycin and Neomycin Class Antibiotics with $\mathrm{N}-1$ Modification
}

Jie Li, Hsiao-Nung Chen, Huiwen Chang, Cheng-Wei Tom Chang*

Department of Chemistry and Biochemistry, Utah State University, 0300 Old Main Hill, Logan, Utah 84322-0300, U.S.A.

Tel: +4357973545. Fax: +4357973390. Email: chang@cc.usu.edu

\section{Supporting Information}

General Experimental Procedure (S2-S15)

Anisotropic Effect from Molecular Modeling (S16)

${ }^{1} \mathrm{H}$ NMR and ${ }^{13} \mathrm{C}$ NMR Spectra for Selected Compounds (S17-S81) 


\section{Experimental Section}

Proton magnetic resonance spectra were recorded using a Jeol 270 or Bruker 400 spectrometers. Chemical shifts were reported as parts per million (ppm) downfield from tetramethylsilane in $\delta$ unit, and coupling constants were given in cycles per second $(\mathrm{Hz})$. Splitting patterns were designed as $\mathrm{s}$, singlet; $\mathrm{d}$, doublet; $\mathrm{t}$, triplet; $\mathrm{q}$, quartet; $\mathrm{m}$, multiplet. ${ }^{13} \mathrm{C}$ spectra were obtained using the Jeol 270 spectrometer at $68 \mathrm{MHz}$ or Bruker 400 spectrometers at $100 \mathrm{MHz}$. Routine 13C NMR spectra were fully decoupled by broadband waltz decoupling. All NMR spectra were recorded at ambient temperature unless otherwise noted. Low-resolution fast-atom bombardment (LRFAB) and High-resolution fast-atom bombardment (HRFAB) or High-resolution MALDI were provided by the Mass Spectrometry Facilities, University of California, Riverside.

Chemical reagents and starting materials were purchased from Aldrich Chemical Co. or Acros Chemical Co. and were used without purification unless otherwise noted. Dichloromethane was distilled over $\mathrm{CaH}_{2}$. Other solvents were used without purification.

3',4'-Di-O-benzyl-1,3,2',6'-tetraazidoneamine (2). To a solution of compound 1 (4.87g, $9.62 \mathrm{mmol})$ in anhydrous THF $(200 \mathrm{~mL}), \mathrm{BnBr}(4.58 \mathrm{~mL}, 38.49 \mathrm{mmol}), \mathrm{NaH}(1.54 \mathrm{~g}$, $38.49 \mathrm{mmol}, 60 \%$ dispersion in mineral oil) and catalytic amount of TBAI were added. The reaction mixture was stirred overnight. The excess $\mathrm{BnBr}$ was quenched by addition of $\mathrm{MeOH}(5 \mathrm{~mL})$ then the reaction mixture was poured into a solution of ice and EtOAc. The aqueous layer was extracted with EtOAc. The combined organic layers were washed with $1 \mathrm{~N} \mathrm{HCl}(\mathrm{aq})$, water, saturated $\mathrm{NaHCO}_{3}(\mathrm{aq})$, and brine, then dried over $\mathrm{Na}_{2} \mathrm{SO}_{4}(\mathrm{~s})$. After removal of the solvent, the crude product was obtained as yellowish oil. To the crude product, $100 \mathrm{~mL}$ mixed solution of dioxane: $\mathrm{H}_{2} \mathrm{O}=1: 1$ was added followed by 45 $\mathrm{mL}$ glacial acetic acid. The resulting mixture solution was refluxed under $60 \sim 65{ }^{\circ} \mathrm{C}$ oilbath for overnight. After completion of the reaction $(\mathrm{Rf}=0.25$, monitored by TLC, EtOAc: Hexane $=35: 65)$, the reaction mixture was quenched with saturated $\mathrm{NaHCO}_{3}(\mathrm{aq})$ and extracted with EtOAc. The organic solution was washed with $1 \mathrm{~N} \mathrm{HCl}(\mathrm{aq})$, water, 
saturated $\mathrm{NaHCO}_{3}(\mathrm{aq})$, brine and dried over $\mathrm{Na}_{2} \mathrm{SO}_{4}(\mathrm{~s})$. After removal of the solvent followed by purification with a gradient column chromatography (Hexane: EtOAc $=80$ : 20 to $40: 60)$, the product was obtained as yellowish oil $(4.52 \mathrm{~g}, 7.51 \mathrm{mmol}, 78 \%){ }^{1} \mathrm{H}$ NMR (400 MHz, CDCl $) \delta 7.3-7.4(\mathrm{~m}, 10 \mathrm{H}), 5.20$ (d, $J=3.6 \mathrm{~Hz}, 1 \mathrm{H}, \mathrm{H}-1$ '), 4.93 (d, $J=$ $10.6 \mathrm{~Hz}, 1 \mathrm{H}, \mathrm{PhCH}_{2} \mathrm{O}$ ), 4.89 (d, $\left.J=11.0 \mathrm{~Hz}, 1 \mathrm{H}, \mathrm{PhCH}_{2} \mathrm{O}\right), 4.88$ (d, $J=10.6 \mathrm{~Hz}, 1 \mathrm{H}$, $\left.\mathrm{PhCH}_{2} \mathrm{O}\right), 4.65\left(\mathrm{~d}, J=11.0 \mathrm{~Hz}, 1 \mathrm{H}, \mathrm{PhCH}_{2} \mathrm{O}\right), 4.15(\mathrm{~m}, 1 \mathrm{H}), 4.00(\mathrm{dd}, J=10.1 \mathrm{~Hz}, J=$ $9.0 \mathrm{~Hz}, 1 \mathrm{H}), 3.6-3.7(\mathrm{~m}, 2 \mathrm{H}), 3.58(\mathrm{dd}, J=13.3 \mathrm{~Hz}, J=2.5 \mathrm{~Hz}, 1 \mathrm{H}), 3.4-3.5(\mathrm{~m}, 4 \mathrm{H})$, $3.2-3.3(\mathrm{~m}, 2 \mathrm{H}), 2.33$ (ddd, $J=13.2 \mathrm{~Hz}, J=3.5 \mathrm{~Hz}, 1 \mathrm{H}, \mathrm{H}-2_{\mathrm{eq}}$ ), 1.50 (ddd, $J=13.2 \mathrm{~Hz}$, $\left.J=12.0 \mathrm{~Hz}, J=12.0 \mathrm{~Hz}, 1 \mathrm{H}, \mathrm{H}-2_{\mathrm{ax}}\right) ;{ }^{13} \mathrm{C}$ NMR $\left(68 \mathrm{MHz}, \mathrm{CDCl}_{3}\right) \delta 137.6(\mathrm{~s}), 137.5(\mathrm{~s})$, 128.6 (s), 128.2 (s), 128.9 (s), 127.7 (s), 127.1 (s), 89.3 (s), 83.3 (s), 80.7 (s), 78.7 (s), $76.2(\mathrm{~s}), 75.7$ (s), 75.5 (s), 75.3 (s), $71.4(\mathrm{~s}), 64.2$ (s), 59.8 (s), 58.9 (s), 51.0 (s), 32.1 (s).

General procedure for diacylation: To the solution of 2 (leq) in anhydrous $\mathrm{CH}_{2} \mathrm{Cl}_{2}$ $(50 \mathrm{ml}), \mathrm{Et}_{3} \mathrm{~N}$ (8eq), DMAP (catalyst amount) and acyl chloride (6eq) were added. The resulting mixture solution was refluxed for overnight. After completion of the reaction, the reaction mixture was quenched with $\mathrm{NaHCO}_{3}(\mathrm{~s})$ and extracted with EtOAc. The organic solution was washed with $1 \mathrm{~N} \mathrm{HCl}(\mathrm{aq})$, water, saturated $\mathrm{NaHCO}_{3}(\mathrm{aq})$, brine and dried over $\mathrm{Na}_{2} \mathrm{SO}_{4}(\mathrm{~s})$. Removal of the solvent followed by purification with a gradient column chromatography (Hexanes:EtOAc $=100: 0$ to $40: 60$ ) afforded the product.

5,6-Di-O-acetyl-3',4'-di-O-benzyl-1,3,2',6'-tetraazidoneamine (3). ' $\mathrm{H}$ NMR (400 MHz, $\left.\mathrm{CDCl}_{3}\right) \delta 7.2-7.4(\mathrm{~m}, 10 \mathrm{H}), 5.16(\mathrm{dd}, J=9.8 \mathrm{~Hz}, J=10.0 \mathrm{~Hz}, 1 \mathrm{H}), 5.13(\mathrm{~d}, J=4.1 \mathrm{~Hz}$, 1H, H-1'), 4.95 (dd, $J=10.0 \mathrm{~Hz}, J=9.9 \mathrm{~Hz}, 1 \mathrm{H}), 4.89$ (d, $J=10.8 \mathrm{~Hz}, 1 \mathrm{H}, \mathrm{PhCH}_{2} \mathrm{O}$ ), $4.88\left(\mathrm{~d}, J=11.1 \mathrm{~Hz}, 1 \mathrm{H}, \mathrm{PhCH}_{2} \mathrm{O}\right), 4.86\left(\mathrm{~d}, J=10.8 \mathrm{~Hz}, 1 \mathrm{H}, \mathrm{PhCH}_{2} \mathrm{O}\right), 4.64(\mathrm{~d}, J=11.1$ $\mathrm{Hz}, 1 \mathrm{H}, \mathrm{PhCH}_{2} \mathrm{O}$ ), 4.24 (m, 1H), 3.96 (dd, $\left.J=10.4 \mathrm{~Hz}, J=8.9 \mathrm{~Hz}, 1 \mathrm{H}\right), 3.6-3.7$ (m, $4 \mathrm{H}), 3.47(\mathrm{~m}, 1 \mathrm{H}), 3.41(\mathrm{dd}, J=13.2 \mathrm{~Hz}, J=4.0 \mathrm{~Hz}, 1 \mathrm{H}), 3.35(\mathrm{dd}, J=10.5 \mathrm{~Hz}, J=3.9$ $\mathrm{Hz}, 1 \mathrm{H}), 2.43$ (ddd, $\left.J=13.3 \mathrm{~Hz}, J=4.6 \mathrm{~Hz}, J=4.6 \mathrm{~Hz}, 1 \mathrm{H}, \mathrm{H}-2_{\mathrm{eq}}\right), 2.11(\mathrm{~s}, 3 \mathrm{H}$, $\mathrm{CH}_{3} \mathrm{CO}_{2}$ ), 2.10 (s, $3 \mathrm{H}, \mathrm{CH}_{3} \mathrm{CO}_{2}$ ), 1.63 (ddd, $J=13.3 \mathrm{~Hz}, J=12.6 \mathrm{~Hz}, J=12.6 \mathrm{~Hz}, 1 \mathrm{H}$, $\left.\mathrm{H}-2_{\mathrm{ax}}\right) ;{ }^{13} \mathrm{C} \mathrm{NMR}\left(100 \mathrm{MHz}, \mathrm{CDCl}_{3}\right) \delta 170.0(\mathrm{~s}), 169.7$ (s), 137.79 (s), $137.76(\mathrm{~s}), 128.8$ (s), $128.7(\mathrm{~s}), 128.3(\mathrm{~s}), 128.2(\mathrm{~s}), 128.0(\mathrm{~s}), 99.3(\mathrm{~s}), 79.7(\mathrm{~s}), 78.9$ (s), 78.7 (s), $75.6(\mathrm{~s})$, 
$75.5(\mathrm{~s}), 74.3(\mathrm{~s}), 73.7(\mathrm{~s}), 71.6(\mathrm{~s}), 63.4(\mathrm{~s}), 58.9(\mathrm{~s}), 57.9(\mathrm{~s}), 51.0(\mathrm{~s}), 32.0(\mathrm{~s}), 20.84(\mathrm{~s}$, $\left.\mathrm{CH}_{3} \mathrm{CO}_{2}\right), 20.75\left(\mathrm{~s}, \mathrm{CH}_{3} \mathrm{CO}_{2}\right)$.

5,6-Di-O-benzoyl-3',4'-di-O-benzyl-1,3,2',6'-tetraazidoneamine (5). ${ }^{1} \mathrm{H}$ NMR (400 $\left.\mathrm{MHz}, \mathrm{CDCl}_{3}\right) \delta 8.0(\mathrm{~m}, 4 \mathrm{H}), 7.3-7.6(\mathrm{~m}, 16 \mathrm{H}), 5.64(\mathrm{dd}, J=9.9 \mathrm{~Hz}, J=9.8 \mathrm{~Hz}, 1 \mathrm{H})$, 5.39 (dd, $J=10.0 \mathrm{~Hz}, J=10.1 \mathrm{~Hz}, 1 \mathrm{H}), 5.12$ (d, $\left.J=3.8 \mathrm{~Hz}, 1 \mathrm{H}, \mathrm{H}-1^{\prime}\right), 4.85$ (d, $J=11.1$ $\left.\mathrm{Hz}, 1 \mathrm{H}, \mathrm{PhCH}_{2} \mathrm{O}\right), 4.80\left(\mathrm{~d}, J=10.6 \mathrm{~Hz}, 1 \mathrm{H}, \mathrm{PhCH}_{2} \mathrm{O}\right), 4.76(\mathrm{~d}, J=10.6 \mathrm{~Hz}, 1 \mathrm{H}$, $\left.\mathrm{PhCH}_{2} \mathrm{O}\right), 4.60\left(\mathrm{~d}, J=11.1 \mathrm{~Hz}, 1 \mathrm{H}, \mathrm{PhCH}_{2} \mathrm{O}\right), 4.22(\mathrm{~m}, 1 \mathrm{H}), 3.8-3.9(\mathrm{~m}, 3 \mathrm{H}), 3.63(\mathrm{~m}$, $1 \mathrm{H}), 3.56(\mathrm{dd}, J=13.2 \mathrm{~Hz}, J=2.4 \mathrm{~Hz}, 1 \mathrm{H}), 3.51(\mathrm{dd}, J=9.6 \mathrm{~Hz}, J=9.4 \mathrm{~Hz}, 1 \mathrm{H}), 3.42$ (dd, $J=13.3 \mathrm{~Hz}, J=4.1 \mathrm{~Hz}, 1 \mathrm{H}), 3.18(\mathrm{dd}, J=10.3 \mathrm{~Hz}, J=3.9 \mathrm{~Hz}, 1 \mathrm{H}), 2.56$ (ddd, $J=$ $13.2 \mathrm{~Hz}, J=4.6 \mathrm{~Hz}, J=4.6 \mathrm{~Hz}, 1 \mathrm{H}, \mathrm{H}-2_{\text {eq }}$ ) $1.8(\mathrm{ddd}, J=13.2 \mathrm{~Hz}, J=12.7 \mathrm{~Hz}, J=12.7$ $\mathrm{Hz}, 1 \mathrm{H}, \mathrm{H}-2_{\mathrm{ax}}$ ); ${ }^{13} \mathrm{C} \mathrm{NMR}\left(100 \mathrm{MHz}, \mathrm{CDCl}_{3}\right) \delta 165.7$ (s), 165.5 (s), 137.8 (s, 2carbons), 133.6 (s), 133.5 (s), 130.0 (s), 129.7 (s), 128.7 (s), 128.64 (s), 128.59 (s), 128.23 (s), $128.20(\mathrm{~s}), 128.1(\mathrm{~s}), 128.0(\mathrm{~s}), 99.1(\mathrm{~s}), 80.2(\mathrm{~s}), 79.0(\mathrm{~s}), 78.7(\mathrm{~s}), 75.6(\mathrm{~s}), 75.4(\mathrm{~s}), 74.2$ (s), $73.6(\mathrm{~s}), 71.6(\mathrm{~s}), 63.5(\mathrm{~s}), 59.4(\mathrm{~s}), 58.4(\mathrm{~s}), 51.1(\mathrm{~s}), 32.4(\mathrm{~s})$.

3',4'-Di- $O$-benzyl-5,6-di-O-(o-chlorobenzoyl)-1,3,2',6'-tetraazidoneamine (6). $\quad{ }^{1} \mathrm{H}$ NMR (400 MHz, $\left.\mathrm{CDCl}_{3}\right) \delta 7.3-8.0(\mathrm{~m}, 18 \mathrm{H}), 5.64(\mathrm{dd}, J=9.8 \mathrm{~Hz}, J=9.8 \mathrm{~Hz}, 1 \mathrm{H}), 5.40$ (dd, $J=10.1 \mathrm{~Hz}, J=10.1 \mathrm{~Hz}, 1 \mathrm{H}), 5.12\left(\mathrm{~d}, J=3.8 \mathrm{~Hz}, 1 \mathrm{H}, \mathrm{H}-\mathrm{l}^{\prime}\right), 4.87$ (d, $J=11.1 \mathrm{~Hz}$, $1 \mathrm{H}, \mathrm{PhCH}_{2} \mathrm{O}$ ), 4.85 (d, $J=10.6 \mathrm{~Hz}, 1 \mathrm{H}, \mathrm{PhCH}_{2} \mathrm{O}$ ), 4.82 (d, $J=10.6 \mathrm{~Hz}, 1 \mathrm{H}, \mathrm{PhCH}_{2} \mathrm{O}$ ), $4.62\left(\mathrm{~d}, J=11.1 \mathrm{~Hz}, 1 \mathrm{H}, \mathrm{PhCH}_{2} \mathrm{O}\right), 4.24(\mathrm{~m}, 1 \mathrm{H}), 3.95(\mathrm{dd}, J=10.3 \mathrm{~Hz}, J=8.9 \mathrm{~Hz}, 1 \mathrm{H})$, $3.86(\mathrm{dd}, J=9.7 \mathrm{~Hz}, J=9.7 \mathrm{~Hz}, 1 \mathrm{H}), 3.78(\mathrm{ddd}, J=12.4 \mathrm{~Hz}, J=10.1 \mathrm{~Hz}, J=4.5 \mathrm{~Hz}$, $1 \mathrm{H}), 3.5-3.7$ (m, 3H), 3.43 (dd, $J=13.3 \mathrm{~Hz}, J=4.0 \mathrm{~Hz}, 1 \mathrm{H}), 3.26$ (dd, $J=10.4 \mathrm{~Hz}, J=$ $3.9 \mathrm{~Hz}, 1 \mathrm{H}), 2.56$ (ddd, $J=13.2 \mathrm{~Hz}, J=4.6 \mathrm{~Hz}, J=4.6 \mathrm{~Hz}, 1 \mathrm{H}, \mathrm{H}-2_{\text {eq }}$ ), 1.82 (ddd, $J=$ $\left.13.3 \mathrm{~Hz}, J=12.6 \mathrm{~Hz}, J=12.6 \mathrm{~Hz}, 1 \mathrm{H}, \mathrm{H}-2_{\mathrm{ax}}\right) ;{ }^{13} \mathrm{C} \mathrm{NMR}\left(100 \mathrm{MHz}, \mathrm{CDCl}_{3}\right) \delta 164.3(\mathrm{~s})$, 163.8 (s), 137.8 (s, 2carbons), 135.0 (s), 134.4 (s), 133.6 (s), 133.3 (s), 132.4 (s), 131.8 (s), 131.7 (s), 131.3 (s), 128.74 (s), 128.66 (s), 128.22 (s), 128.12 (s), 128.0 (s), 127.0 (s), $126.9(\mathrm{~s}), 99.2(\mathrm{~s}), 80.1(\mathrm{~s}), 79.0(\mathrm{~s}), 78.7(\mathrm{~s}), 75.6(\mathrm{~s}), 75.4(\mathrm{~s}), 74.3(\mathrm{~s}), 73.7(\mathrm{~s}), 71.6(\mathrm{~s})$, $63.5(\mathrm{~s}), 59.3(\mathrm{~s}), 58.4(\mathrm{~s}), 51.1(\mathrm{~s}), 32.3(\mathrm{~s})$. 
3',4'-Di- $O$-benzyl-5,6-di- $O$-( $p$-chlorobenzoyl)-1,3,2',6'-tetraazidoneamine $\quad$ (7). $\quad{ }^{1} \mathrm{H}$ NMR (400 MHz, $\left.\mathrm{CDCl}_{3}\right) \delta 7.8-7.9(\mathrm{~m}, 4 \mathrm{H}), 7.3-7.4(\mathrm{~m}, 14 \mathrm{H}), 5.59$ (dd, $J=9.9 \mathrm{~Hz}, J=$ $9.9 \mathrm{~Hz}, 1 \mathrm{H}), 5.35(\mathrm{dd}, J=9.9 \mathrm{~Hz}, J=10.2 \mathrm{~Hz}, 1 \mathrm{H}), 5.07$ (d, $\left.J=3.6 \mathrm{~Hz}, 1 \mathrm{H}, \mathrm{H}-1^{\prime}\right), 4.85$ $\left(\mathrm{d}, J=11.2 \mathrm{~Hz}, 1 \mathrm{H}, \mathrm{PhCH}_{2} \mathrm{O}\right), 4.81$ (d, $\left.J=10.6 \mathrm{~Hz}, 1 \mathrm{H}, \mathrm{PhCH}_{2} \mathrm{O}\right), 4.76$ (d, $J=10.6 \mathrm{~Hz}$, $\left.1 \mathrm{H}, \mathrm{PhCH}_{2} \mathrm{O}\right), 4.60\left(\mathrm{~d}, J=11.2 \mathrm{~Hz}, 1 \mathrm{H}, \mathrm{PhCH}_{2} \mathrm{O}\right), 4.20(\mathrm{~m}, 1 \mathrm{H}), 3.8-3.9(\mathrm{~m}, 3 \mathrm{H}), 3.5-$ $3.6(\mathrm{~m}, 3 \mathrm{H}), 3.42(\mathrm{dd}, J=13.3 \mathrm{~Hz}, J=3.9 \mathrm{~Hz}, 1 \mathrm{H}), 3.17(\mathrm{dd}, J=10.3 \mathrm{~Hz}, J=3.9 \mathrm{~Hz}$, $1 \mathrm{H}), 2.56$ (ddd, $J=13.3 \mathrm{~Hz}, J=4.6 \mathrm{~Hz}, J=4.6 \mathrm{~Hz}, 1 \mathrm{H}, \mathrm{H}-2_{\mathrm{eq}}$ ), 1.79 (ddd, $J=13.3 \mathrm{~Hz}$, $\left.J=12.5 \mathrm{~Hz}, J=12.5 \mathrm{~Hz}, 1 \mathrm{H}, \mathrm{H}-2_{\mathrm{ax}}\right) ;{ }^{13} \mathrm{C} \mathrm{NMR}\left(100 \mathrm{MHz}, \mathrm{CDCl}_{3}\right) \delta 164.9$ (s), 164.7 (s), 140.4 (s), 140.2 (s), 137.74 (s), 137.70 (s), 131.4 (s), 131.0 (s), 129.2 (s), 129.1 (s), 128.8 (s), 128.7 (s), 128.3 (s), 128.1 (s), 128.0 (s), 127.6 (s), 127.1 (s), 99.2 (s), 80.1 (s), 79.0 (s), 78.7 (s), 75.6 (s), 75.4 (s), 74.4 (s), 73.9 (s), 71.7 (s), 63.4 (s), 59.3 (s), 58.2 (s), 51.1 (s), 32.3 (s);MALDI Calcd for $\mathrm{C}_{40} \mathrm{H}_{36} \mathrm{Cl}_{2} \mathrm{~N}_{12} \mathrm{O}_{8} \mathrm{Na}\left([\mathrm{M}+\mathrm{Na}]^{+}\right)$m/e 905.2048; measure m/e 905.2065.

6-O-Benzoyl-3',4'-di- $O$-benzyl-1,3,2',6'-tetraazidoneamine (4). To the solution of 2 (2.00 g, $3.30 \mathrm{mmol})$ and DIPEA $(0.76 \mathrm{~mL}, 4.62 \mathrm{mmol})$ in anhydrous $\mathrm{CH}_{2} \mathrm{Cl}_{2}(50 \mathrm{~mL})$, $\mathrm{BzCl}(0.46 \mathrm{~mL}, 3.96 \mathrm{mmol})$ were added at $-50^{\circ} \mathrm{C}$. The reaction mixture was stirred allowing the reaction temperature to warm up to $0^{\circ} \mathrm{C}$ and quenched with $\mathrm{NaHCO}_{3}(\mathrm{~s})$ after completion of the reaction $(\mathrm{Rf}=0.4$, monitored by TLC, EtOAc: Hexane $=25: 75)$. After removal of the solvent, the reaction mixture was diluted with EtOAc. The organic layers were washed with $1 \mathrm{~N} \mathrm{HCl}(\mathrm{aq})$, saturated $\mathrm{NaHCO}_{3}(\mathrm{aq})$, and brine, then dried over $\mathrm{Na}_{2} \mathrm{SO}_{4}(\mathrm{~s})$. Removal of the solvent followed by purification with gradient column chromatography (Hexanes:EtOAc $=100: 0$ to $60: 40)$ afforded the product $(0.95 \mathrm{~g}, 1.35$ mmol, 41\%). ${ }^{1} \mathrm{H}$ NMR (400 MHz, $\left.\mathrm{CDCl}_{3}\right) \delta 8.0-8.1(\mathrm{~m}, 2 \mathrm{H}), 7.3-7.6(\mathrm{~m}, 13 \mathrm{H}), 5.26(\mathrm{~d}$, $\left.J=3.7 \mathrm{~Hz}, 1 \mathrm{H}, \mathrm{H}^{\prime} 1^{\prime}\right), 5.21$ (dd, $\left.J=10.1 \mathrm{~Hz}, J=9.9 \mathrm{~Hz}, 1 \mathrm{H}\right), 4.91$ (d, $J=10.6 \mathrm{~Hz}, 1 \mathrm{H}$, $\left.\mathrm{PhCH}_{2} \mathrm{O}\right), 4.89$ (d, $\left.J=11.1 \mathrm{~Hz}, 1 \mathrm{H}, \mathrm{PhCH}_{2} \mathrm{O}\right), 4.86\left(\mathrm{~d}, J=10.6 \mathrm{~Hz}, 1 \mathrm{H}, \mathrm{PhCH}_{2} \mathrm{O}\right), 4.65$ $\left(\mathrm{d}, J=11.1 \mathrm{~Hz}, 1 \mathrm{H}, \mathrm{PhCH}_{2} \mathrm{O}\right), 4.15(\mathrm{~m}, 1 \mathrm{H}), 3.98(\mathrm{dd}, J=10.1 \mathrm{~Hz}, J=9.0 \mathrm{~Hz}, 1 \mathrm{H}), 3.81$ $(\mathrm{m}, 1 \mathrm{H}), 3.69(\mathrm{ddd}, J=12.5 \mathrm{~Hz}, J=10.1 \mathrm{~Hz}, J=4.6 \mathrm{~Hz}, 1 \mathrm{H}), 3.63(\mathrm{dd}, J=10.2 \mathrm{~Hz}, J=$ $10.1 \mathrm{~Hz}, 1 \mathrm{H}), 3.5-3.6(\mathrm{~m}, 2 \mathrm{H}), 3.47(\mathrm{dd}, J=9.8 \mathrm{~Hz}, J=8.7 \mathrm{~Hz}, 1 \mathrm{H}), 3.3-3.4(\mathrm{~m}, 2 \mathrm{H})$, 2.46 (ddd, $J=13.2 \mathrm{~Hz}, J=4.5 \mathrm{~Hz}, J=4.5 \mathrm{~Hz}, 1 \mathrm{H}, \mathrm{H}-2_{\mathrm{eq}}$ ), 1.71 (ddd, $J=13.2 \mathrm{~Hz}, J=$ $\left.12.4 \mathrm{~Hz}, J=12.4 \mathrm{~Hz}, 1 \mathrm{H}, \mathrm{H}-2_{\mathrm{ax}}\right) ;{ }^{13} \mathrm{C} \mathrm{NMR}\left(100 \mathrm{MHz}, \mathrm{CDCl}_{3}\right) \delta 166.0$ (s), 137.7 (s), 
$137.6(\mathrm{~s}), 133.7(\mathrm{~s}), 130.2(\mathrm{~s}), 129.4(\mathrm{~s}), 128.8(\mathrm{~s}), 128.72(\mathrm{~s}), 128.69(\mathrm{~s}), 128.3(\mathrm{~s}), 128.2(\mathrm{~s})$, 128.0(s), $99.8(\mathrm{~s}), 84.4(\mathrm{~s}), 81.0(\mathrm{~s}), 78.9(\mathrm{~s}), 75.9(\mathrm{~s}), 75.5(\mathrm{~s}), 75.43(\mathrm{~s}), 74.8(\mathrm{~s}), 71.6(\mathrm{~s})$, $64.5(\mathrm{~s}), 58.9(\mathrm{~s}), 58.5(\mathrm{~s}), 51.2(\mathrm{~s}), 32.4(\mathrm{~s})$.

General procedure for one-pot selective Staudinger reaction: The diacylated compound (1 eq) was placed in a flask with BOC-ON (2.4eq) ([2-(tertbutoxycarbonyloxyimino)-2-phenylacetonitrile], under $\mathrm{N}_{2}$ and dissolved in $10 \mathrm{ml}$ anhydrous toluene. The reaction was cooled to $-78^{\circ} \mathrm{C}$ and $1.0 \mathrm{M}$ trimethylphosphine (1.1 eq) in toluene was added. The reaction was kept at $-78^{\circ} \mathrm{C}$ for 30 minutes, then was allowed to warm to $10^{\circ} \mathrm{C}$. The reaction was quenched with phophate buffer $(\mathrm{PH}=7.4)$ and was concentrated. The residue was extracted with EtOAc. The organic layer was washed with $1 \mathrm{~N} \mathrm{HCl}(\mathrm{aq}), \mathrm{H}_{2} \mathrm{O}$, saturated $\mathrm{NaHCO}_{3}(\mathrm{aq})$, and brine, then dried over $\mathrm{Na}_{2} \mathrm{SO}_{4}(\mathrm{~s})$. Removal of the solvent followed by purification with gradient column chromatography (Hexanes:EtOAc $=100: 0$ to 40:60) afforded the product.

\section{5,6-Di- $O$-acetyl-3',4'-di- $O$-benzyl-1- $N$-tert-butoxycarbonyl-3,2',6'-triazidoneamine}

(8a). ${ }^{1} \mathrm{H}$ NMR (400 MHz, $\left.\mathrm{CDCl}_{3}\right) \delta 7.3-7.4(\mathrm{~m}, 10 \mathrm{H}), 5.22(\mathrm{dd}, J=9.7 \mathrm{~Hz}, J=9.7 \mathrm{~Hz}$, $1 \mathrm{H}), 5.11\left(\mathrm{~d}, J=3.6 \mathrm{~Hz}, 1 \mathrm{H}, \mathrm{H}-1^{\prime}\right), 4.8-4.9\left(\mathrm{~m}, 3 \mathrm{H}, \mathrm{PhCH}_{2} \mathrm{O}\right), 4.80(\mathrm{dd}, J=10.3 \mathrm{~Hz}, J=$ $10.3 \mathrm{~Hz}, 1 \mathrm{H}), 4.63\left(\mathrm{~d}, J=11.1 \mathrm{~Hz}, 1 \mathrm{H}, \mathrm{PhCH}_{2} \mathrm{O}\right), 4.60(\mathrm{~m}, 1 \mathrm{H}), 4.26(\mathrm{~m}, 1 \mathrm{H}), 3.98$ (dd, $J$ $=9.4 \mathrm{~Hz}, J=9.8 \mathrm{~Hz}, 1 \mathrm{H}), 3.83(\mathrm{~m}, 1 \mathrm{H}), 3.4-3.6(\mathrm{~m}, 3 \mathrm{H}), 3.4(\mathrm{dd}, J=13.4 \mathrm{~Hz}, J=$ $3.9 \mathrm{~Hz}, 1 \mathrm{H}), 3.36(\mathrm{dd}, J=10.4 \mathrm{~Hz}, J=3.6 \mathrm{~Hz}, 1 \mathrm{H}), 2.51\left(\mathrm{~m}, 1 \mathrm{H}, \mathrm{H}-2_{\mathrm{eq}}\right), 2.10(\mathrm{~s}, 3 \mathrm{H}$, $\mathrm{CH}_{3} \mathrm{CO}_{2}$ ), 2.05 (s, $3 \mathrm{H}, \mathrm{CH}_{3} \mathrm{CO}_{2}$ ), 1.51 (ddd, $J=13.1 \mathrm{~Hz}, J=12.7 \mathrm{~Hz}, J=12.7 \mathrm{~Hz}, 1 \mathrm{H}$, $\left.\mathrm{H}-2_{\mathrm{ax}}\right), 1.44(\mathrm{~s}, 9 \mathrm{H}) ;{ }^{13} \mathrm{C}$ NMR (100 MHz, $\left.\mathrm{CDCl}_{3}\right) \delta 171.3$ (s), 169.3 (s), $155.2(\mathrm{~s}), 137.8$ (s, 2carbons), 128.74 (s), 128.7 (s), 128.2(s), 128.16(s), 128.0(s), 99.3 (s), 80.5 (s), 79.8 (s), $79.2(\mathrm{~s}), 78.9(\mathrm{~s}), 75.6(\mathrm{~s}), 75.4(\mathrm{~s}), 73.6(\mathrm{~s}), 71.5(\mathrm{~s}), 63.5(\mathrm{~s}), 59.2(\mathrm{~s}), 51.1(\mathrm{~s}), 49.1$ (s), 33.5 (s), 28.5 (s), 20.9 (s, $\left.\mathrm{CH}_{3} \mathrm{CO}_{2}\right), 20.8\left(\mathrm{~s}, \mathrm{CH}_{3} \mathrm{CO}_{2}\right.$ ).

\section{5,6-Di- $O$-acetyl-3',4'-di- $O$-benzyl-3- $N$-tert-butoxycarbonyl-1,2',6'-triazidoneamine}

(9a). ${ }^{1} \mathrm{H}$ NMR $\left(400 \mathrm{MHz}, \mathrm{CDCl}_{3}\right) \delta 7.2-7.4(\mathrm{~m}, 10 \mathrm{H}), 5.18(\mathrm{dd}, J=9.6 \mathrm{~Hz}, J=9.6 \mathrm{~Hz}$, $1 \mathrm{H}), 5.05\left(\mathrm{~d}, J=3.8 \mathrm{~Hz}, 1 \mathrm{H}, \mathrm{H}-1^{\prime}\right), 4.91(\mathrm{dd}, J=10.1 \mathrm{~Hz}, J=9.9 \mathrm{~Hz}, 1 \mathrm{H}), 4.8-$ $4.9\left(\mathrm{~m}, 3 \mathrm{H}, \mathrm{PhCH} \mathrm{H}_{2} \mathrm{O}\right), 4.80(\mathrm{~m}, \mathrm{H}), 4.59\left(\mathrm{~d}, J=11.3 \mathrm{~Hz}, 1 \mathrm{H}, \mathrm{PhCH}_{2} \mathrm{O}\right), 4.02(\mathrm{~m}, 1 \mathrm{H})$, 
$3.91(\mathrm{dd}, J=10.2 \mathrm{~Hz}, J=9.0 \mathrm{~Hz}, 1 \mathrm{H}), 3.75(\mathrm{~m}, 1 \mathrm{H}), 3.50-3.70(\mathrm{~m}, 4 \mathrm{H}), 3.35(\mathrm{dd}, J=$ $10.7 \mathrm{~Hz}, J=3.9 \mathrm{~Hz}, 1 \mathrm{H}), 2.46\left(\mathrm{~m}, 1 \mathrm{H}, \mathrm{H}-2_{\mathrm{eq}}\right), 2.10$ (s, 3H, $\left.\mathrm{CH}_{3} \mathrm{CO}_{2}\right), 2.09$ (s, 3H, $\left.\mathrm{CH}_{3} \mathrm{CO}_{2}\right), 1.42(\mathrm{~s}, 9 \mathrm{H}), 1.37\left(\mathrm{~m}, 1 \mathrm{H}, \mathrm{H}-2_{\mathrm{ax}}\right) ;{ }^{13} \mathrm{C} \mathrm{NMR}\left(100 \mathrm{MHz}, \mathrm{CDCl}_{3}\right) \delta 170.2(\mathrm{~s})$, 169.7 (s), 155.0 (s), 137.8 (s), 137.7 (s), 128.7 (s), 128.6 (s), 128.25 (s), 128.18 (s), $128.14(\mathrm{~s}), 127.8(\mathrm{~s}), 99.9(\mathrm{~s}), 80.8(\mathrm{~s}), 80.0(\mathrm{~s}), 79.6(\mathrm{~s}), 79.0(\mathrm{~s}), 75.7(\mathrm{~s}), 75.3(\mathrm{~s}), 74.7$ (s), $74.3(\mathrm{~s}), 71.1(\mathrm{~s}), 63.3(\mathrm{~s}), 58.2(\mathrm{~s}), 51.4(\mathrm{~s}), 49.5$ (s), 34.0 (s), 28.5 (s), 20.9 (s, $\left.\mathrm{CH}_{3} \mathrm{CO}_{2}\right), 20.8\left(\mathrm{~s}, \mathrm{CH}_{3} \mathrm{CO}_{2}\right)$.

\section{3',4'-Di- $\mathrm{O}$-benzyl-5,6-di-O-benzoyl-1- $\mathrm{N}$-tert-butoxycarbonyl -3,2',6'-triazidoneamine}

(8c). ${ }^{1} \mathrm{H}$ NMR (400 MHz, $\left.\mathrm{CDCl}_{3}\right) \delta 7.8-7.9(\mathrm{~m}, 4 \mathrm{H}), 7.3-7.4(\mathrm{~m}, 16 \mathrm{H}), 5.71$ (dd, $J=$ $9.8 \mathrm{~Hz}, J=9.8 \mathrm{~Hz}, 1 \mathrm{H}, \mathrm{H}-5), 5.21$ (dd, $J=10.3 \mathrm{~Hz}, J=10.3 \mathrm{~Hz}, 1 \mathrm{H}, \mathrm{H}-6), 5.13$ (d, $J=$ $\left.3.8 \mathrm{~Hz}, 1 \mathrm{H}, \mathrm{H}-1^{\prime}\right), 4.85$ (d, $\left.J=11.2 \mathrm{~Hz}, 1 \mathrm{H}, \mathrm{PhCH}_{2} \mathrm{O}\right), 4.81$ (d, $J=10.6 \mathrm{~Hz}, 1 \mathrm{H}, \mathrm{PhCH}_{2} \mathrm{O}$ ), $4.77\left(\mathrm{~d}, J=10.6 \mathrm{~Hz}, 1 \mathrm{H}, \mathrm{PhCH}_{2} \mathrm{O}\right), 4.60\left(\mathrm{~d}, J=11.2 \mathrm{~Hz}, 1 \mathrm{H}, \mathrm{PhCH}_{2} \mathrm{O}\right), 4.26(\mathrm{~m}, 1 \mathrm{H})$, $4.05(\mathrm{~m}, 1 \mathrm{H}), 3.92(\mathrm{dd}, J=10.0 \mathrm{~Hz}, J=9.1 \mathrm{~Hz}, 1 \mathrm{H}), 3.83(\mathrm{dd}, J=9.7 \mathrm{~Hz}, J=9.7 \mathrm{~Hz}$, $1 \mathrm{H}), 3.62$ (ddd, $J=12.3 \mathrm{~Hz}, J=10.1 \mathrm{~Hz}, J=4.7 \mathrm{~Hz}, 1 \mathrm{H}$ ), 3.56 (dd, $J=13.3 \mathrm{~Hz}, J=2.5$ $\mathrm{Hz}, 1 \mathrm{H}$ ), 3.51 (dd, $J=9.3 \mathrm{~Hz}, J=9.5 \mathrm{~Hz}, 1 \mathrm{H}), 3.43$ (dd, $J=13.3 \mathrm{~Hz}, J=4.0 \mathrm{~Hz}, 1 \mathrm{H}$ ), $3.19(\mathrm{dd}, J=10.3 \mathrm{~Hz}, J=3.7 \mathrm{~Hz}, 1 \mathrm{H}), 2.63(\mathrm{ddd}, J=12.9 \mathrm{~Hz}, J=3.9 \mathrm{~Hz}, J=3.9 \mathrm{~Hz}$, $1 \mathrm{H}, \mathrm{H}-2_{\mathrm{eq}}$ ) 1.69 (ddd, $\left.J=12.9 \mathrm{~Hz}, J=12.6 \mathrm{~Hz}, J=12.6 \mathrm{~Hz}, 1 \mathrm{H}, \mathrm{H}-2_{\mathrm{ax}}\right), 1.28(\mathrm{~s}, 9 \mathrm{H})$; ${ }^{13} \mathrm{C} \mathrm{NMR}\left(100 \mathrm{MHz}, \mathrm{CDCl}_{3}\right) \delta 167.1$ (s), 165.3 (s), 155.2 (s), 137.8 (s, 2carbons), 133.7 (s), $133.4(\mathrm{~s}), 130.2(\mathrm{~s}), 129.7$ (s), 128.7 (s), 128.6 (s), 128.5 (s), 128.2 (s), 128.1 (s), $128.0(\mathrm{~s}), 99.1(\mathrm{~s}), 80.4(\mathrm{~s}), 80.3(\mathrm{~s}), 79.6(\mathrm{~s}), 78.7(\mathrm{~s}), 75.7(\mathrm{~s}), 75.3(\mathrm{~s}), 74.1(\mathrm{~s}), 73.8(\mathrm{~s})$, $71.5(\mathrm{~s}), 63.6(\mathrm{~s}), 59.6(\mathrm{~s}), 51.1(\mathrm{~s}), 49.6(\mathrm{~s}), 33.7(\mathrm{~s}), 28.3(\mathrm{~s})$.

\section{3',4'-Di-O-benzyl-1- $N$-tert-butoxycarbonyl-5,6-di-O-(o-chlorobenzoyl)-3,2',6'-}

triazidoneamine (8d). ${ }^{1} \mathrm{H}$ NMR $\left(400 \mathrm{MHz}, \mathrm{CDCl}_{3}\right) \delta 7.3-8.0(\mathrm{~m}, 18 \mathrm{H}), 5.69(\mathrm{dd}, J=$ $9.8 \mathrm{~Hz}, J=9.7 \mathrm{~Hz}, 1 \mathrm{H}), 5.33(\mathrm{dd}, J=10.4 \mathrm{~Hz}, J=10.2 \mathrm{~Hz}, 1 \mathrm{H}), 5.11(\mathrm{~d}, J=3.8 \mathrm{~Hz}, 1 \mathrm{H}$, H-1'), $4.8-4.9\left(\mathrm{~m}, 3 \mathrm{H}, \mathrm{PhCH}_{2} \mathrm{O}\right), 4.62\left(\mathrm{~d}, J=11.2 \mathrm{~Hz}, 1 \mathrm{H}, \mathrm{PhCH}_{2} \mathrm{O}\right), 4.27(\mathrm{~m}, 1 \mathrm{H}), 4.03$ (m, 1H), $3.98(\mathrm{dd}, J=10.3 \mathrm{~Hz}, J=9.0 \mathrm{~Hz}, 1 \mathrm{H}), 3.83(\mathrm{dd}, J=9.7 \mathrm{~Hz}, J=9.7 \mathrm{~Hz}, 1 \mathrm{H})$, $3.50-3.70(\mathrm{~m}, 3 \mathrm{H}), 3.42(\mathrm{dd}, J=13.3 \mathrm{~Hz}, J=3.9 \mathrm{~Hz}, 1 \mathrm{H}), 3.25(\mathrm{dd}, J=10.4 \mathrm{~Hz}, J=3.8$ $\mathrm{Hz}, 1 \mathrm{H}$ ), 2.58 (ddd, $J=13.0 \mathrm{~Hz}, J=4.3 \mathrm{~Hz}, J=4.3 \mathrm{~Hz}, 1 \mathrm{H}, \mathrm{H}-2_{\mathrm{eq}}$ ), 1.71 (ddd, $J=13.0$ $\left.\mathrm{Hz}, J=12.6 \mathrm{~Hz}, J=12.6 \mathrm{~Hz}, 1 \mathrm{H}, \mathrm{H}-2_{\mathrm{ax}}\right), 1.36(\mathrm{~s}, 9 \mathrm{H}) ;{ }^{13} \mathrm{C} \mathrm{NMR}\left(100 \mathrm{MHz}, \mathrm{CDCl}_{3}\right)$ 
$\delta 165.5$ (s), 164.2 (s), 155.4 (s), 137.8 (s, 2carbons), 134.5 (s), 134.4 (s), 133.4 (s), 133.3 (s), 132.3 (s), 132.0 (s), 131.7 (s), 131.5 (s), 129.3 (s), 128.7 (s), 128.65 (s), 128.3 (s), $128.2(\mathrm{~s}), 128.0$ (s), 127.0 (s), 126.5 (s), 99.2 (s), 80.7 (s), 80.1 (s), 79.4 (s), 78.8 (s), 75.7 (s), $75.4(\mathrm{~s}), 74.1(\mathrm{~s}), 74.0$ (s), 71.5 (s), 63.5 (s), 59.5 (s), 51.1 (s), 49.5 (s), 33.7 (s), 28.4 $(\mathrm{s})$.

\section{3',4'-Di-O-benzyl-1,3-di- $N$-tert-butoxycarbonyl-5,6-di-O-(o-chlorobenzoyl)-2',6'-}

diazidoneamine (9d). ${ }^{1} \mathrm{H}$ NMR $\left(400 \mathrm{MHz}, \mathrm{CDCl}_{3}\right) \delta 7.2-8.0$ (m, 18H), $5.68(\mathrm{dd}, J=9.5$ $\mathrm{Hz}, J=9.5 \mathrm{~Hz}, 1 \mathrm{H}$ ), 5.30 (dd, $J=10.4 \mathrm{~Hz}, J=9.4 \mathrm{~Hz}, 1 \mathrm{H}), 5.03$ (d, $\left.J=3.8 \mathrm{~Hz}, 1 \mathrm{H}, \mathrm{H}-1^{\prime}\right)$, $4.87\left(\mathrm{~d}, J=11.3 \mathrm{~Hz}, 1 \mathrm{H}, \mathrm{PhCH}_{2} \mathrm{O}\right), 4.85\left(\mathrm{~d}, J=10.6 \mathrm{~Hz}, 1 \mathrm{H}, \mathrm{PhCH}_{2} \mathrm{O}\right), 4.82(\mathrm{~d}, J=10.6$ $\left.\mathrm{Hz}, 1 \mathrm{H}, \mathrm{PhCH}_{2} \mathrm{O}\right), 4.70(\mathrm{~m}, 1 \mathrm{H}), 4.56\left(\mathrm{~d}, J=11.3 \mathrm{~Hz}, 1 \mathrm{H}, \mathrm{PhCH}_{2} \mathrm{O}\right), 4.00(\mathrm{~m}, 1 \mathrm{H}), 3.92$ $(\mathrm{dd}, J=10.1 \mathrm{~Hz}, J=9.2 \mathrm{~Hz}, 1 \mathrm{H}), 3.81(\mathrm{~m}, 1 \mathrm{H}), 3.63(\mathrm{~m}, 1 \mathrm{H}), 3.49(\mathrm{~m}, 1 \mathrm{H}), 3.34(\mathrm{dd}, J=$ $13.3 \mathrm{~Hz}, J=4.6 \mathrm{~Hz}, 1 \mathrm{H}), 3.23$ (dd, $J=10.4 \mathrm{~Hz}, J=3.8 \mathrm{~Hz}, 1 \mathrm{H}), 3.13(\mathrm{~m}, 1 \mathrm{H}), 2.53(\mathrm{~m}$, $\left.1 \mathrm{H}, \mathrm{H}-2_{\mathrm{eq}}\right), 1.44(\mathrm{~s}, 9 \mathrm{H}), 1.33\left(\mathrm{~m}, 1 \mathrm{H}, \mathrm{H}-2_{\mathrm{ax}}\right), 1.33(\mathrm{~s}, 9 \mathrm{H}) ;{ }^{13} \mathrm{C}$ NMR $\left(100 \mathrm{MHz}, \mathrm{CDCl}_{3}\right)$ $\delta 165.3$ (s), 164.1 (s), 155.1 (s, 2carbons), 137.9 (s), 137.8 (s), 134.6 (s), 134.3 (s), 133.2 (s), 133.1 (s), 132.4 (s), 132.1 (s), 131.6 (s), 131.1 (s), 130.0 (s), 129.8 (s), 128.6 (s), $128.3(\mathrm{~s}), 128.1(\mathrm{~s}), 127.8(\mathrm{~s}), 126.9(\mathrm{~s}), 99.8(\mathrm{~s}), 80.8(\mathrm{~s}), 80.04(\mathrm{~s}), 80.01$ (s), $78.8(\mathrm{~s})$, 75.7 (s), $75.2(\mathrm{~s}), 74.6$ (s), 74.5 (s), 71.1 (s), 63.5 (s), 51.3 (s), 50.5 (s), 49.6 (s), 39.2 (s), $29.9(\mathrm{~s}), 28.6(\mathrm{~s}), 28.4(\mathrm{~s})$.

\section{3',4'-Di-O-benzyl-1- $N$-tert-butoxycarbonyl-5,6-di- $O$-(p-chlorobenzoyl)-3,2',6-}

triazidoneamine (8e). ${ }^{1} \mathrm{H}$ NMR $\left(400 \mathrm{MHz}, \mathrm{CDCl}_{3}\right) \delta 7.8-7.9(\mathrm{~m}, 4 \mathrm{H}), 7.3-$ $7.4(\mathrm{~m}, 14 \mathrm{H}), 5.65(\mathrm{dd}, J=9.8 \mathrm{~Hz}, J=9.8 \mathrm{~Hz}, 1 \mathrm{H}, \mathrm{H}-5), 5.17(\mathrm{dd}, J=10.5 \mathrm{~Hz}, J=10.2$ $\mathrm{Hz}, 1 \mathrm{H}, \mathrm{H}-6), 5.07$ (d, $J=3.8 \mathrm{~Hz}, 1 \mathrm{H}, \mathrm{H}-1$ '), 4.85 (d, $\left.J=11.2 \mathrm{~Hz}, 1 \mathrm{H}, \mathrm{PhCH}_{2} \mathrm{O}\right), 4.82$ (d, $\left.J=10.6 \mathrm{~Hz}, 1 \mathrm{H}, \mathrm{PhCH}_{2} \mathrm{O}\right), 4.77\left(\mathrm{~d}, J=10.6 \mathrm{~Hz}, 1 \mathrm{H}, \mathrm{PhCH}_{2} \mathrm{O}\right), 4.60$ (d, $J=11.2 \mathrm{~Hz}, 1 \mathrm{H}$, $\mathrm{PhCH}_{2} \mathrm{O}$ ), 4.25 (m, 1H, H-5'), 4.05 (m, 1H, H-1), 3.91 (dd, $J=10.2 \mathrm{~Hz}, J=9.0 \mathrm{~Hz}, 1 \mathrm{H}$, H-3), 3.55 (dd, $\left.J=13.3 \mathrm{~Hz}, J=2.5 \mathrm{~Hz}, 1 \mathrm{H}, \mathrm{H}-6^{\prime}\right), 3.50$ (dd, $J=9.0 \mathrm{~Hz}, J=9.1 \mathrm{~Hz}, 1 \mathrm{H}$, H-4'), 3.42 (dd, $J=13.3 \mathrm{~Hz}, J=4.1 \mathrm{~Hz}, 1 \mathrm{H}, \mathrm{H}-6^{\prime}$ ), 3.19 (dd, $J=10.3 \mathrm{~Hz}, J=3.8 \mathrm{~Hz}, 1 \mathrm{H}$, H-2'), 2.60 (ddd, $J=13.0 \mathrm{~Hz}, J=4.2 \mathrm{~Hz}, J=4.2 \mathrm{~Hz}, 1 \mathrm{H}, \mathrm{H}-2_{\text {eq }}$ ), 1.68 (ddd, $J=13.0 \mathrm{~Hz}$, $\left.J=12.6 \mathrm{~Hz}, J=12.6 \mathrm{~Hz}, 1 \mathrm{H}, \mathrm{H}-2_{\mathrm{ax}}\right), 1.29(\mathrm{~s}, 9 \mathrm{H}) ;{ }^{13} \mathrm{C} \mathrm{NMR}\left(100 \mathrm{MHz}, \mathrm{CDCl}_{3}\right) \delta 166.0$ (s), 164.5 (s), 155.2 (s), 140.4 (s), 140.1 (s), 137.79 (s), 137.76 (s), 131.5 (s), 131.0 (s), 
129.2 (s), 129.0 (s), 128.7 (s), 128.6 (s), 128.23 (s), 128.17 (s), 128.11 (s), 128.0 (s), 127.8 (s), 127.2 (s), 99.2 (s), 80.5 (s), 80.1 (s), 79.5 (s), 78.7 (s), 75.6 (s), 75.4 (s), 74.4 (s), 74.1 (s), 71.6 (s), 63.5 (s), 59.5 (s), 51.1 (s), 49.3 (s), 33.6 (s), 28.3 (s);MALDI Calcd for $\mathrm{C}_{45} \mathrm{H}_{46} \mathrm{Cl}_{2} \mathrm{~N}_{10} \mathrm{O}_{10} \mathrm{Na}\left([\mathrm{M}+\mathrm{Na}]^{+}\right) \mathrm{m} / \mathrm{e}$ 979.2668; measure m/e 979.2649.

\section{3',4'-Di-O-benzyl-3- $N$-tert-butoxycarbonyl-5,6-di- $O$-( $p$-chlorobenzoyl)-1,2',6-}

triazidoneamine (9e). ${ }^{1} \mathrm{H}$ NMR $\left(400 \mathrm{MHz}, \mathrm{CDCl}_{3}\right) \delta 7.8-7.9(\mathrm{~m}, 4 \mathrm{H}), 7.2-$ $7.3(\mathrm{~m}, 14 \mathrm{H}), 5.66(\mathrm{dd}, J=9.6 \mathrm{~Hz}, J=9.4 \mathrm{~Hz}, 1 \mathrm{H}), 5.20(\mathrm{dd}, J=9.9 \mathrm{~Hz}, J=10.2 \mathrm{~Hz}, 1 \mathrm{H})$, 5.01 (d, $J=3.8 \mathrm{~Hz}, 1 \mathrm{H}, \mathrm{H}-1$ '), 4.89 (m, 1H), 4.85 (d, $\left.J=11.3 \mathrm{~Hz}, 1 \mathrm{H}, \mathrm{PhCH}_{2} \mathrm{O}\right), 4.79$ (s, 2H, $\mathrm{PhCH}_{2} \mathrm{O}$ ), $4.71(\mathrm{~m}, 1 \mathrm{H}), 4.56\left(\mathrm{~d}, J=11.3 \mathrm{~Hz}, 1 \mathrm{H}, \mathrm{PhCH}_{2} \mathrm{O}\right.$ ), $4.02(\mathrm{~m}, 1 \mathrm{H}), 3.87$ (dd, $J=10.0 \mathrm{~Hz}, J=9.1 \mathrm{~Hz}, 1 \mathrm{H}), 3.85(\mathrm{~m}, 1 \mathrm{H}), 3.62(\mathrm{~m}, 1 \mathrm{H}), 3.47$ (dd, $J=9.1 \mathrm{~Hz}, J=9.1 \mathrm{~Hz}$, 1H), 3.37 (dd, $J=13.0 \mathrm{~Hz}, J=4.2 \mathrm{~Hz}, 1 \mathrm{H}), 3.20(\mathrm{dd}, J=10.3 \mathrm{~Hz}, J=3.7 \mathrm{~Hz}, 1 \mathrm{H}), 2.53$ (m, 1H, H-2 eq, 1.44 (s, 9H), 1.29 (m, 1H, H-2 $\left.2_{\mathrm{ax}}\right), 1.25$ (s, 9H); ${ }^{13} \mathrm{C}$ NMR (100 MHz, $\left.\mathrm{CDCl}_{3}\right) \delta 166.0$ (s), 164.6 (s), 155.1 (s, 2carbons), 140.1 (s), 139.7 (s), 137.9 (s), 137.7 (s), $131.6(\mathrm{~s}), 130.9$ (s), 129.1 (s), 128.9 (s), 128.6 (s), 128.1 (s), 127.8 (s), 127.5 (s), 99.0 (s), 80.6 (s), 80.1 (s), 78.8 (s), 75.7 (s), 75.3 (s), 75.1 (s), 74.7 (s), 71.1 (s), 63.5 (s), 51.4 (s), $50.5(\mathrm{~s}), 49.4(\mathrm{~s}), 35.1(\mathrm{~s}), 29.9(\mathrm{~s}), 28.6(\mathrm{~s}), 28.3(\mathrm{~s})$.

3',4'-Di-O-benzyl-1- $N$-tert-butoxycarbonyl-3,2',6'-triazidoneamine (10). A solution of $8 \mathrm{e}(0.71 \mathrm{~g}, 0.74 \mathrm{mmol})$ and $\mathrm{NaOMe}(0.5 \mathrm{M}, 4.45 \mathrm{ml}, 2.22 \mathrm{mmol})$ in anhydrous $\mathrm{MeOH} / \mathrm{THF}$ $(10 \mathrm{~mL} / 2 \mathrm{~mL})$ was stirred at room temperature till the complete consumption of starting material (ca. 2 hours). $(\mathrm{Rf}=0.25$, monitored by TLC, EtOAc: Hexane $=35: 65)$. Then Amberlite $120 \mathrm{H}^{+}$was added to quench the reaction. The reaction mixture was filtered through Celite and washed with EtOAc and $\mathrm{MeOH}$. Removal of solvent followed by purification with a gradient column chromatography (Hexanes : EtOAc $=70: 30$ to 30:70) afforded compound $10(0.27 \mathrm{~g}, 0.39 \mathrm{mmol}, 53.5 \%)$ along with compound $11(0.04 \mathrm{~g}$, $0.066 \mathrm{mmol}, 8 \%) .{ }^{1} \mathrm{H}$ NMR (400 MHz, $\left.\mathrm{CDCl}_{3}\right) \delta 7.3-7.4(\mathrm{~m}, 10 \mathrm{H}), 5.28(\mathrm{~d}, J=3.4 \mathrm{~Hz}$, 1H, H-1'), 4.93 (d, $J=10.7 \mathrm{~Hz}, 1 \mathrm{H}, \mathrm{PhCH}_{2} \mathrm{O}$ ), 4.89 (d, $J=10.7 \mathrm{~Hz}, 1 \mathrm{H}, \mathrm{PhCH}_{2} \mathrm{O}$ ), 4.89 (d, $J=11.1 \mathrm{~Hz}, 1 \mathrm{H}, \mathrm{PhCH}_{2} \mathrm{O}$ ), 4.65 (d, $J=11.1 \mathrm{~Hz}, 1 \mathrm{H}, \mathrm{PhCH}_{2} \mathrm{O}$ ), 4.17 (ddd, $J=9.8 \mathrm{~Hz}$, $J=4.0 \mathrm{~Hz}, J=3.3 \mathrm{~Hz}, 1 \mathrm{H}), 4.02(\mathrm{dd}, J=9.6 \mathrm{~Hz}, J=9.4 \mathrm{~Hz}, 1 \mathrm{H}), 3.5-3.6(\mathrm{~m}, 5 \mathrm{H}), 3.42$ (dd, $J=13.3 \mathrm{~Hz}, J=4.0 \mathrm{~Hz}, 1 \mathrm{H}), 3.3-4.0(\mathrm{~m}, 3 \mathrm{H}), 2.51(\mathrm{ddd}, J=12.9 \mathrm{~Hz}, J=3.7 \mathrm{~Hz}$, 
$\left.J=3.7 \mathrm{~Hz}, 1 \mathrm{H}, \mathrm{H}-2_{\mathrm{eq}}\right), 1.47$ (s, 9H), $1.42\left(\mathrm{~m}, 1 \mathrm{H}, \mathrm{H}-2_{\mathrm{ax}}\right) ;{ }^{13} \mathrm{C} \mathrm{NMR}\left(100 \mathrm{MHz}, \mathrm{CDCl}_{3}\right)$ $\delta 156.4(\mathrm{~s}), 137.8$ (s), 137.7 (s), 128.76 (s), 128.72 (s), 128.28 (s), 128.23 (s), 128.0 (s), 99.4 (s), 83.7 (s), 80.9 (s), 80.6 (s), 78.9 (s), 75.9 (s), 75.4 (s), 74.8 (s, 2 carbons), 71.4 (s), 64.4 (s), 59.4 (s), 51.2 (s), 50.8 (s), 33.3 (s), 28.5 (s); MALDI Calcd for $\mathrm{C}_{31} \mathrm{H}_{40} \mathrm{~N}_{10} \mathrm{O}_{8} \mathrm{Na}$ $\left([\mathrm{M}+\mathrm{Na}]^{+}\right) \mathrm{m} / \mathrm{e} 703.2923$; measure $\mathrm{m} / \mathrm{e} 703.2922$.

3',4'-Di-O-benzyl-3-N-tert-butoxycarbonyl-1,2',6'-triazidoneamine (11). ${ }^{1} \mathrm{H} \quad \mathrm{NMR}$ $\left(400 \mathrm{MHz}, \mathrm{CDCl}_{3}\right) \delta 7.3-7.4$ (m, 10H), 5.06 (d, $\left.J=3.6 \mathrm{~Hz}, 1 \mathrm{H}, \mathrm{H}-1^{\prime}\right), 4.90$ (d, $J=10.6$ $\left.\mathrm{Hz}, 1 \mathrm{H}, \mathrm{PhCH}_{2} \mathrm{O}\right), 4.89\left(\mathrm{~d}, J=11.2 \mathrm{~Hz}, 1 \mathrm{H}, \mathrm{PhCH}_{2} \mathrm{O}\right), 4.87(\mathrm{~d}, J=10.6 \mathrm{~Hz}, 1 \mathrm{H}$, $\mathrm{PhCH}_{2} \mathrm{O}$ ), 4.63 (d, $J=11.2 \mathrm{~Hz}, 1 \mathrm{H}, \mathrm{PhCH}_{2} \mathrm{O}$ ), 3.97 (m, 2H), $3.4-3.7$ (m, 7H), 3.39 (dd, $J=13.4 \mathrm{~Hz}, J=3.4 \mathrm{~Hz}, 1 \mathrm{H}), 3.21$ (dd, $J=10.3 \mathrm{~Hz}, J=8.9 \mathrm{~Hz}, 1 \mathrm{H}), 2.47\left(\mathrm{~m}, 1 \mathrm{H}, \mathrm{H}-2_{\mathrm{eq}}\right.$ ), $1.44(\mathrm{~s}, 9 \mathrm{H}), 1.28\left(\mathrm{~m}, 1 \mathrm{H}, \mathrm{H}-2_{\mathrm{ax}}\right) ;{ }^{13} \mathrm{C} \mathrm{NMR}\left(100 \mathrm{MHz}, \mathrm{CDCl}_{3}\right) \delta 155.2(\mathrm{~s}), 137.8(\mathrm{~s})$, $137.5(\mathrm{~s}), 128.7$ (s), 128.3 (s), 128.2 (s), 127.7 (s), 100.2 (s), 85.6 (s), 80.8 (s), 80.6 (s), 78.6 (s), 76.5 (s), 76.0 (s), 75.7 (s), 75.3 (s), 71.5 (s), 64.4 (s), 60.3 (s), 50.9 (s), 49.5 (s), $33.9(\mathrm{~s}), 28.6(\mathrm{~s})$

6-O-Benzoyl-3',4'-di-O-benzyl-1- $N$-tert-butoxycarbonyl-3,2',6'-triazidoneamine (12). Please refer to the procedure for synthesis of compound $4 .{ }^{1} \mathrm{H}$ NMR $\left(400 \mathrm{MHz}, \mathrm{CDCl}_{3}\right)$ $\delta 8.0-8.1(\mathrm{~m}, 2 \mathrm{H}), 7.3-7.5(\mathrm{~m}, 13 \mathrm{H}), 5.23(\mathrm{~d}, J=3.6 \mathrm{~Hz}, 1 \mathrm{H}, \mathrm{H}-1$ '), $5.03(\mathrm{dd}, J=10.2$ $\mathrm{Hz}, J=10.1 \mathrm{~Hz}, 1 \mathrm{H}), 4.92\left(\mathrm{~d}, J=10.7 \mathrm{~Hz}, 1 \mathrm{H}, \mathrm{PhCH}_{2} \mathrm{O}\right), 4.89(\mathrm{~d}, J=11.1 \mathrm{~Hz}, 1 \mathrm{H}$, $\left.\mathrm{PhCH}_{2} \mathrm{O}\right), 4.87\left(\mathrm{~d}, J=10.7 \mathrm{~Hz}, 1 \mathrm{H}, \mathrm{PhCH}_{2} \mathrm{O}\right), 4.65\left(\mathrm{~d}, J=11.1 \mathrm{~Hz}, 1 \mathrm{H}, \mathrm{PhCH}_{2} \mathrm{O}\right), 4.17$ (m, 1H), $4.00(\mathrm{dd}, J=9.9 \mathrm{~Hz}, J=9.2 \mathrm{~Hz}, 1 \mathrm{H}), 3.87(\mathrm{dd}, J=9.0 \mathrm{~Hz}, J=9.1 \mathrm{~Hz}, 1 \mathrm{H}), 3.6$ -3.7 (m, 4H), $3.4-3.5$ (m, 3H), 2.51 (ddd, $J=13.2 \mathrm{~Hz}, J=3.9 \mathrm{~Hz}, J=3.9 \mathrm{~Hz}, 1 \mathrm{H}, \mathrm{H}-$ 2 eq $), 1.56\left(\mathrm{~m}, 1 \mathrm{H}, \mathrm{H}-2_{\mathrm{ax}}\right), 1.27$ (s, 9H); ${ }^{13} \mathrm{C} \mathrm{NMR}\left(100 \mathrm{MHz}, \mathrm{CDCl}_{3}\right) \delta 167.2(\mathrm{~s}), 155.5(\mathrm{~s})$, $137.8(\mathrm{~s}), 137.7(\mathrm{~s}), 133.6(\mathrm{~s}), 130.3(\mathrm{~s}), 129.6(\mathrm{~s}), 128.8(\mathrm{~s}), 128.7(\mathrm{~s}), 128.6(\mathrm{~s}), 128.3(\mathrm{~s})$, $128.0(\mathrm{~s}), 99.8(\mathrm{~s}), 84.9(\mathrm{~s}), 81.0(\mathrm{~s}), 80.2(\mathrm{~s}), 78.9(\mathrm{~s}), 75.9(\mathrm{~s}), 75.4(\mathrm{~s}), 75.3(\mathrm{~s}), 74.8(\mathrm{~s})$, 71.5 (s), 64.5 (s), 59.2 (s), 51.2 (s), 49.2 (s), 33.7 (s), 28.3 (s); MALDI Calcd for $\mathrm{C}_{38} \mathrm{H}_{44} \mathrm{~N}_{10} \mathrm{O} 9 \mathrm{Na}\left([\mathrm{M}+\mathrm{Na}]^{+}\right) \mathrm{m} / \mathrm{e} 807.3185$; measure m/e 807.3165.

6-O-(4-Azido-2,3-di-O-benzyl-4,6-dideoxy- $\alpha$-D-glucopyranosyl)-3',4'-di-O-benzyl-1$N$-tert-butoxycarbonyl-3,2',6'-triazidoneamine (13). Please refer to refernce $9 .{ }^{1} \mathrm{H}$ 
NMR (400 MHz, CDCl $) \delta 7.3-7.4(\mathrm{~m}, 20 \mathrm{H}), 5.48(\mathrm{~d}, J=3.6 \mathrm{~Hz}, 1 \mathrm{H}, \mathrm{H}-1), 4.96(\mathrm{~d}, J=$ $\left.10.4 \mathrm{~Hz}, 1 \mathrm{H}, \mathrm{PhCH}_{2} \mathrm{O}\right), 4.85-4.93\left(\mathrm{~m}, 6 \mathrm{H}, \mathrm{PhCH}_{2} \mathrm{O}\right), 4.87(\mathrm{~d}, J=10.6 \mathrm{~Hz}, 1 \mathrm{H}$, $\mathrm{PhCH}_{2} \mathrm{O}$ ), 4.78 (d, $\left.J=3.2 \mathrm{~Hz}, 1 \mathrm{H}, \mathrm{H}-1^{\prime}\right), 4.67$ (dd, $\left.J=11.4 \mathrm{~Hz}, J=11.0 \mathrm{~Hz}, 1 \mathrm{H}\right), 4.24$ (m, $1 \mathrm{H}), 4.00(\mathrm{dd}, J=9.6 \mathrm{~Hz}, J=9.4 \mathrm{~Hz}, 1 \mathrm{H}), 3.7-3.8(\mathrm{~m}, 2 \mathrm{H}), 3.3-3.7(\mathrm{~m}, 8 \mathrm{H}), 3.1-$ $3.2(\mathrm{~m}, 2 \mathrm{H}), 2.87\left(\mathrm{~m}, 1 \mathrm{H}, \mathrm{H}-2_{\mathrm{eq}}\right), 1.42(\mathrm{~s}, 9 \mathrm{H}), 1.41\left(\mathrm{~m}, 1 \mathrm{H}, \mathrm{H}-2_{\mathrm{ax}}\right), 1.31$ (d, J=6.2 Hz, 3H, H-6"); ${ }^{13} \mathrm{C}$ NMR (100 MHz, $\mathrm{CDCl}_{3}$ ) $\delta 156.5$ (s), 137.95 (s), 137.87 (s), 137.2 (s), $129.06(\mathrm{~s}), 128.87$ (s), 128.82 (s), 128.70 (s), 128.52 (s), 128.26 (s), 128.17 (s), 128.15 (s), 127.98 (s), 99.4 (s), 98.7 (s), 84.4 (s), 81.6 (s), 80.6 (s), 80.4 (s), 79.83 (s), 79.76 (s), 78.9 (s), 76.7 (s), 76.1 (s), 75.8 (s), 75.3 (s), 74.6 (s), 71.2 (s), 68.3 (s), 67.9 (s), 64.1 (s), 59.3 (s), 51.2 (s), 50.1 (s), 32.9 (s), 28.7 (s), 18.7 (s); MALDI Calcd for $\mathrm{C}_{51} \mathrm{H}_{61} \mathrm{~N}_{13} \mathrm{O}_{11} \mathrm{Na}$ $\left([\mathrm{M}+\mathrm{Na}]^{+}\right) \mathrm{m} / \mathrm{e} 1054.4506 ;$ measure $\mathrm{m} / \mathrm{e} 1054.4557$.

\section{6-O-(3-Azido-2-O-benzyl-3,6-dideoxy- $\alpha$-D-glucopyranosyl)-3',4'-di- $O$-benzyl-1- $N$ -} tert-butoxycarbonyl-3,2',6'-triazidoneamine (14). Please refer to refernce $8 .{ }^{1} \mathrm{H}$ NMR (400 MHz, $\left.\mathrm{CDCl}_{3}\right) \delta 7.3-7.4(\mathrm{~m}, 15 \mathrm{H}), 5.48\left(\mathrm{~d}, J=3.7 \mathrm{~Hz}, 1 \mathrm{H}, \mathrm{H}-1^{\prime}\right), 4.8-4.9(\mathrm{~m}, 4 \mathrm{H}$, $\mathrm{PhCH}_{2} \mathrm{O}$ ), 4.76 (d, $J=3.4 \mathrm{~Hz}, 1 \mathrm{H}, \mathrm{H}-1$ "), 4.65 (d, $\left.J=11.8 \mathrm{~Hz}, 1 \mathrm{H}, \mathrm{PhCH}_{2} \mathrm{O}\right), 4.64$ (d, $J=$ $\left.11.2 \mathrm{~Hz}, 1 \mathrm{H}, \mathrm{PhCH}_{2} \mathrm{O}\right), 4.23(\mathrm{~m}, 1 \mathrm{H}), 3.98(\mathrm{dd}, J=10.1 \mathrm{~Hz}, J=9.0 \mathrm{~Hz}, 1 \mathrm{H}), 3.90(\mathrm{~m}$, $1 \mathrm{H}), 3.76$ (dd, $J=9.9 \mathrm{~Hz}, J=9.8 \mathrm{~Hz}, 1 \mathrm{H}), 3.3-3.7$ (m, 9H), 3.08 (dd, $J=10.3 \mathrm{~Hz}, J=$ $9.2 \mathrm{~Hz}, 1 \mathrm{H}), 3.04(\mathrm{dd}, J=9.5 \mathrm{~Hz}, J=9.6 \mathrm{~Hz}, 1 \mathrm{H}), 2.78(\mathrm{ddd}, J=13.1 \mathrm{~Hz}, J=3.9 \mathrm{~Hz}, J=$ $3.9 \mathrm{~Hz}, 1 \mathrm{H}, \mathrm{H}-2_{\mathrm{eq}}$ ), 1.56 (m, 1H, H-2 $2_{\mathrm{ax}}$ ), 1.51 (s, 9H), 1.27 (d, $J=6.1 \mathrm{~Hz}, 3 \mathrm{H}, \mathrm{H}-6$ "); ${ }^{13} \mathrm{C}$ NMR (100 MHz, CDCl $\left.{ }_{3}\right) \delta 156.5$ (s), 137.9 (s, 2 carbons), 136.9 (s), 129.1 (s), 129.0 (s), $128.95(\mathrm{~s}), 128.7(\mathrm{~s}), 128.5(\mathrm{~s}), 128.24(\mathrm{~s}), 128.16(\mathrm{~s}), 128.0(\mathrm{~s}), 98.7$ (s), $98.5(\mathrm{~s}), 84.6(\mathrm{~s})$, 81.5 (s), 80.4 (s), 79.9 (s), 79.2 (s), 78.8 (s), 76.7 (s), 75.8 (s), 75.3 (s), 74.3 (s, 2 carbons), $71.2(\mathrm{~s}), 69.0(\mathrm{~s}), 65.6(\mathrm{~s}), 64.0(\mathrm{~s}), 59.2(\mathrm{~s}), 51.2(\mathrm{~s}), 49.9(\mathrm{~s}), 32.9(\mathrm{~s}), 28.6(\mathrm{~s}), 17.9(\mathrm{~s})$.

General procedure for $\boldsymbol{t}$-Boc deprotection after glycosylation reaction: A solution of glycosylated compound $(13,14$ or 17$)$ in dichloromethane was treated with $99 \%$ trifluoroacetic acid and the reaction mixture was stirred for $3 \mathrm{hrs}$ at room temperature. The reaction mixture was quenched with triethylamine and concentrated to dryness as crude product which was directly used for peptide coupling. 
6-O-(4-Azido-2,3-di- $O$-benzyl-4,6-dideoxy- $\alpha$-D-glucopyranosyl)-3',4'-di- $O$-benzyl-1$N$-[(S)-4-(benzyloxycarbonylamino)-2-hydroxybutanoyl]-3,2',6'-triazidoneamine

(15). A mixture of (S)-N-Carbobenzyloxyl-4-amino-2-hydroxybutyric acid (0.1g, $0.39 \mathrm{mmol})$ and $N$-hydroxysuccinimide $(0.1 \mathrm{~g}, 0.79 \mathrm{mmol})$, DCC $(0.1 \mathrm{~g}, 0.47 \mathrm{mmol})$ in anhydrous THF $(5 \mathrm{ml})$ was stirred at room temperature under nitrogen for $2 \mathrm{hrs}$. This solution was directly mixed with triethylamine $(3 \mathrm{ml})$ and the crude product which was deprotected of $t$-Boc group under nitrogen. The reaction was stirred at room temperature for overnight. After completion of the reaction $(\mathrm{Rf}=0.35$, monitored by TLC, EtOAc: Hexane $=50: 50$ ), the reaction mixture was concentrated and extracted with EtOAc. The organic layer was washed with $1 \mathrm{~N} \mathrm{HCl}(\mathrm{aq})$, saturated $\mathrm{NaHCO}_{3}(\mathrm{aq})$, and brine, then dried over $\mathrm{Na}_{2} \mathrm{SO}_{4}(\mathrm{~s})$. Removal of the solvent followed by purification with gradient column chromatography (Hexanes:EtOAc $=50: 50$ to $20: 80)$ afforded the product $(0.04 \mathrm{~g}, 0.03$ mmol, 58.9\%). ${ }^{1} \mathrm{H}$ NMR (400 MHz, $\left.\mathrm{CDCl}_{3}\right) \delta 7.3-7.4(\mathrm{~m}, 25 \mathrm{H}), 5.54(\mathrm{~d}, J=3.7 \mathrm{~Hz}, 1 \mathrm{H}$, $\left.\mathrm{H}-1^{\prime}\right), 5.0$ - 5.1 (m, 2H, $\left.\mathrm{PhCH}_{2} \mathrm{O}\right), 4.8-4.9$ (m, 7H, $\mathrm{PhCH}_{2} \mathrm{O}, \mathrm{H}-1$ '), 4.65 (d, J= $11.1 \mathrm{~Hz}$, $\left.1 \mathrm{H}, \mathrm{PhCH}_{2} \mathrm{O}\right), 4.64\left(\mathrm{~d}, J=11.8 \mathrm{~Hz}, 1 \mathrm{H}, \mathrm{PhCH}_{2} \mathrm{O}\right), 4.2-4.3(\mathrm{~m}, 2 \mathrm{H}), 4.00(\mathrm{dd}, J=10.1$ $\mathrm{Hz}, J=9.1 \mathrm{~Hz}, 1 \mathrm{H}), 3.7-3.8(\mathrm{~m}, 3 \mathrm{H}), 3.64(\mathrm{dd}, J=8.5 \mathrm{~Hz}, J=8.5 \mathrm{~Hz}, 1 \mathrm{H}), 3.4-3.6(\mathrm{~m}$, 9H), 3.29 (dd, $J=9.9 \mathrm{~Hz}, J=9.5 \mathrm{~Hz}, 1 \mathrm{H}), 3.09(\mathrm{dd}, J=9.9 \mathrm{~Hz}, J=9.7 \mathrm{~Hz}, 1 \mathrm{H}), 2.63$ (ddd, $\left.J=13.2 \mathrm{~Hz}, J=3.8 \mathrm{~Hz}, J=3.8 \mathrm{~Hz}, 1 \mathrm{H}, \mathrm{H}-2_{\mathrm{eq}}\right), 1.6-1.8(\mathrm{~m}, 2 \mathrm{H}), 1.39$ (m, $1 \mathrm{H}, \mathrm{H}-$ $2_{\text {ax }}$ ), 1.28 (d, J=6.2 Hz, 3H, H-6"); ${ }^{13} \mathrm{C}$ NMR (100 MHz, $\left.\mathrm{CDCl}_{3}\right) \delta 174.5$ (s), 158.1 (s), 138.1 (s), 137.98 (s), 137.95 (s), 137.7 (s), 136.3 (s), 129.1 (s), 128.84 (s), 128.79 (s), 128.73 (s), 128.5 (s), 128.32 (s), 128.29 (s), 128.27 (s), 128.17 (s), 128.0 (s), 98.66 (s), 98.56 (s), 84.0 (s), 80.9 (s), 80.2 (s, 2 carbons), 79.8 (s), 78.9 (s), 76.7 (s), 75.8 (s), 75.7 (s), 75.4 (s), 74.3 (s), 71.2 (s), 69.4 (s), 68.1 (s, 2 carbons), 67.4 (s), 63.9 (s), 59.2 (s), 51.3 (s), 48.4 (s), 37.1 (s), 35.1 (s), 32.3 (s), 18.6 (s); MALDI Calcd for $\mathrm{C}_{58} \mathrm{H}_{66} \mathrm{~N}_{14} \mathrm{O}_{13} \mathrm{Na}$ $\left([\mathrm{M}+\mathrm{Na}]^{+}\right) \mathrm{m} / \mathrm{e} 1189.4826$; measure m/e 1189.4783 .

6-O-(3-Azido-2-O-benzyl-3,6-dideoxy- $\alpha$-D-glucopyranosyl)-3',4'-di- $O$-benzyl-1- $N$ [(S)-4-(benzyloxycarbonylamino)-2-hydroxybutanoyl]-3,2',6'-triazidoneamine (16). A mixture of $(S)$ - $N$-Carbobenzyloxyl-4-amino-2-hydroxybutyric acid $(0.144 \mathrm{~g}, 0.57 \mathrm{mmol})$ and $\operatorname{EDC}(0.12 \mathrm{~g}, 0.60 \mathrm{mmol})$, HOBT $(0.08 \mathrm{~g}, 0.60 \mathrm{mmol}), \mathrm{Et}_{3} \mathrm{~N}(3 \mathrm{ml})$ in anhydrous DMF $(5 \mathrm{ml})$ was stirred under nitrogen at room temperature for overnight. After completion of 
the reaction $(\mathrm{Rf}=0.4$, monitored by TLC, EtOAc: Hexane $=50: 50)$, the reaction mixture was concentrated and extracted with EtOAc. The organic layer was washed with $1 \mathrm{~N}$ $\mathrm{HCl}(\mathrm{aq})$, saturated $\mathrm{NaHCO}_{3}(\mathrm{aq})$, and brine, then dried over $\mathrm{Na}_{2} \mathrm{SO}_{4}(\mathrm{~s})$. Removal of the solvent followed by purification with gradient column chromatography (Hexanes:EtOAc $=60: 40$ to $20: 80)$ afforded the product $(0.11 \mathrm{~g}, 0.10 \mathrm{mmol}, 60 \%) .{ }^{1} \mathrm{H}$ NMR $(400 \mathrm{MHz}$, $\left.\mathrm{CDCl}_{3}\right) \delta 7.3-7.4(\mathrm{~m}, 2 \mathrm{H}), 5.50\left(\mathrm{~d}, J=3.7 \mathrm{~Hz}, 1 \mathrm{H}, \mathrm{H}-1\right.$ '), $5.0-5.1\left(\mathrm{~m}, 2 \mathrm{H}, \mathrm{PhCH}_{2} \mathrm{O}\right)$, $4.8-4.9$ (m, 5H, $\mathrm{PhCH}_{2} \mathrm{O}, \mathrm{H}-1$ "), $4.64\left(\mathrm{~d}, J=11.1 \mathrm{~Hz}, 1 \mathrm{H}, \mathrm{PhCH}_{2} \mathrm{O}\right), 4.62(\mathrm{~d}, J=11.7$ $\left.\mathrm{Hz}, 1 \mathrm{H}, \mathrm{PhCH}_{2} \mathrm{O}\right), 4.24(\mathrm{~m}, 1 \mathrm{H}), 4.07(\mathrm{~m}, 1 \mathrm{H}), 3.98(\mathrm{dd}, J=10.2 \mathrm{~Hz}, J=8.9 \mathrm{~Hz}, 1 \mathrm{H})$, $3.87(\mathrm{~m}, 1 \mathrm{H}), 3.76(\mathrm{dd}, J=9.9 \mathrm{~Hz}, J=9.8 \mathrm{~Hz}, 1 \mathrm{H}), 3.5-3.7(\mathrm{~m}, 4 \mathrm{H}), 3.3-3.5(\mathrm{~m}, 6 \mathrm{H})$, $3.1-3.2(\mathrm{~m}, 2 \mathrm{H}), 3.01(\mathrm{dd}, J=9.5 \mathrm{~Hz}, J=9.6 \mathrm{~Hz}, 1 \mathrm{H}), 2.57$ (ddd, $J=12.7 \mathrm{~Hz}, J=3.9$ $\left.\mathrm{Hz}, J=3.9 \mathrm{~Hz}, 1 \mathrm{H}, \mathrm{H}-2_{\mathrm{eq}}\right), 1.7-2.0(\mathrm{~m}, 2 \mathrm{H}), 1.32\left(\mathrm{~m}, 1 \mathrm{H}, \mathrm{H}-2_{\mathrm{ax}}\right), 1.26(\mathrm{~d}, J=5.9 \mathrm{~Hz}, 3 \mathrm{H}$, H-6"); ${ }^{13} \mathrm{C}$ NMR (100 MHz, $\left.\mathrm{CDCl}_{3}\right) \delta 174.0$ (s), 158.1 (s), 137.95 (s), 137.90 (s), 137.3 (s), 136.4 (s), 129.1 (s), 129.0 (s), 128.8 (s), 128.72 (s), 128.69 (s), 128.5 (s), 128.24 (s), $128.18(\mathrm{~s}), 128.0(\mathrm{~s}), 98.8$ (s), 97.7 (s), 83.8 (s), 81.2 (s), 80.3 (s), 79.3 (s), 78.9 (s), 77.1 (s), $75.7(\mathrm{~s}), 75.4(\mathrm{~s}), 74.5(\mathrm{~s}), 74.2(\mathrm{~s}), 71.2(\mathrm{~s}), 69.2(\mathrm{~s}), 67.3(\mathrm{~s}), 67.1(\mathrm{~s}), 65.1(\mathrm{~s}), 63.9$ (s), $59.2(\mathrm{~s}), 51.3(\mathrm{~s}), 48.1(\mathrm{~s}), 37.1(\mathrm{~s}), 34.9(\mathrm{~s}), 32.5(\mathrm{~s}), 17.8(\mathrm{~s})$.

\section{6-O-(4-Amino-4,6-dideoxy- $\alpha$-D-glucopyranosyl)-1- $N$ - $[($ S)-4-amino-2-}

hydroxybutanoyl]neamine (JLN005). Please refer to refernce 9. ${ }^{1} \mathrm{H}$ NMR $(270 \mathrm{MHz}$, $\mathrm{D}_{2} \mathrm{O}$ ) (chloride salt) $\delta 5.96\left(\mathrm{~d}, J=3.6 \mathrm{~Hz}, 1 \mathrm{H}, \mathrm{H}-1^{\prime}\right), 5.12\left(\mathrm{~d}, J=4.0 \mathrm{~Hz}, 1 \mathrm{H}, \mathrm{H}-1^{\prime \prime}\right), 4.37$ $(\mathrm{m}, 1 \mathrm{H}), 4.28(\mathrm{dd}, J=8.9 \mathrm{~Hz}, J=3.6 \mathrm{~Hz}, 1 \mathrm{H}), 4.0-4.1(\mathrm{~m}, 4 \mathrm{H}), 3.8-3.9(\mathrm{~m}, 3 \mathrm{H}), 3.5-$ $3.6(\mathrm{~m}, 5 \mathrm{H}), 3.29(\mathrm{dd}, J=13.5 \mathrm{~Hz}, J=7.2 \mathrm{~Hz}, 1 \mathrm{H}), 3.1-3.2(\mathrm{~m}, 2 \mathrm{H}), 2.98(\mathrm{dd}, J=10.2$ $\mathrm{Hz}, J=10.2 \mathrm{~Hz}, 1 \mathrm{H}), 2.21\left(\mathrm{~m}, 1 \mathrm{H}, \mathrm{H}-2_{\mathrm{eq}}\right), 2.0-2.2(\mathrm{~m}, 2 \mathrm{H}), 1.85(\mathrm{ddd}, J=12.6 \mathrm{~Hz}, J=$ $\left.12.9 \mathrm{~Hz}, J=12.9 \mathrm{~Hz}, 1 \mathrm{H}, \mathrm{H}-2_{\mathrm{ax}}\right), 1.31(\mathrm{~d}, J=6.3 \mathrm{~Hz}, 3 \mathrm{H}, \mathrm{H}-6 ") ;{ }^{13} \mathrm{C}$ NMR $(100 \mathrm{MHz}$, $\mathrm{D}_{2} \mathrm{O}$ ) (chloride salt) $\delta 175.6(\mathrm{~s}), 99.4(\mathrm{~s}), 96.0(\mathrm{~s}), 80.7(\mathrm{~s}), 78.6(\mathrm{~s}), 75.0(\mathrm{~s}), 71.8(\mathrm{~s})$, $71.0(\mathrm{~s}), 69.9(\mathrm{~s}), 69.5(\mathrm{~s}), 69.1(\mathrm{~s}), 68.7(\mathrm{~s}), 65.4(\mathrm{~s}), 57.2$ (s), 53.9 (s), 49.0 (s, 2 carbons), $40.5(\mathrm{~s}), 37.3(\mathrm{~s}), 31.0(\mathrm{~s}), 30.8(\mathrm{~s}), 17.0(\mathrm{~s})$.

\section{6-O-(3-Amino-3,6-dideoxy- $\alpha$-D-glucopyranosyl)-1- $N$-[(S)-4-amino-2-}

hydroxybutanoyl]neamine (JLN027). Please refer to refernce 9. 'H NMR (400 MHz, $\mathrm{D}_{2} \mathrm{O}$ ) (chloride salt) $\delta 5.93\left(\mathrm{~d}, J=3.8 \mathrm{~Hz}, 1 \mathrm{H}, \mathrm{H}-1^{\prime}\right), 5.07(\mathrm{~d}, J=3.7 \mathrm{~Hz}, 1 \mathrm{H}, \mathrm{H}-1 "), 4.25$ 
$(\mathrm{dd}, J=9.4 \mathrm{~Hz}, J=3.7 \mathrm{~Hz}, 1 \mathrm{H}), 3.9-4.1(\mathrm{~m}, 5 \mathrm{H}), 3.8-3.9(\mathrm{~m}, 2 \mathrm{H}), 3.78(\mathrm{~m}, 1 \mathrm{H}), 3.4-$ $3.5(\mathrm{~m}, 4 \mathrm{H}), 3.3-3.4(\mathrm{~m}, 3 \mathrm{H}), 3.15(\mathrm{dd}, J=7.0 \mathrm{~Hz}, J=7.1 \mathrm{~Hz}, 2 \mathrm{H}), 2.21(\mathrm{ddd}, J=12.8$ $\left.\mathrm{Hz}, J=4.6 \mathrm{~Hz}, J=4.6 \mathrm{~Hz}, 1 \mathrm{H}, \mathrm{H}-2_{\mathrm{eq}}\right), 1.9-2.2(\mathrm{~m}, 2 \mathrm{H}), 1.83(\mathrm{ddd}, J=12.8 \mathrm{~Hz}, J=$ $\left.12.3 \mathrm{~Hz}, J=12.3 \mathrm{~Hz}, 1 \mathrm{H}, \mathrm{H}-2_{\mathrm{ax}}\right), 1.23$ (d, $\left.J=6.2 \mathrm{~Hz}, 3 \mathrm{H}, \mathrm{H}-6 "\right) ;{ }^{13} \mathrm{C}$ NMR (100 MHz, $\mathrm{D}_{2} \mathrm{O}$ ) (chloride salt) $\delta 175.7$ (s), 98.1 (s), 96.1 (s), 80.3 (s), 78.4 (s), 74.9 (s), 71.1 (s), 70.8 (s), 69.8 (s), 69.5 (s), 68.7 (s), 68.5 (s), 68.4 (s), 55.3 (s), 53.7 (s), 48.9 (s, 2 carbons), 40.3 (s), 37.2 (s), $31.1(\mathrm{~s}), 30.6$ (s), 16.4 (s).

\section{5-O-(4-Azido-4,6-dideoxy- $\beta$-D-glucopyranosyl)-3',4'-di-O-benzyl-1- $N$-tert-}

butoxycarbonyl-3,2',6'-triazidoneamine (17). Please refer to refernce 8. ${ }^{1} \mathrm{H}$ NMR (400 $\left.\mathrm{MHz} \mathrm{CDCl}_{3}\right) \delta 7.3-7.4(\mathrm{~m}, 10 \mathrm{H}), 6.00\left(\mathrm{~d}, J=3.8 \mathrm{~Hz}, 1 \mathrm{H}, \mathrm{H}-1^{\prime}\right), 4.90(\mathrm{~d}, J=11.1 \mathrm{~Hz}$, $2 \mathrm{H}, \mathrm{PhCH}_{2} \mathrm{O}$ ), 4.87 (d, $J=10.7 \mathrm{~Hz}, 1 \mathrm{H}, \mathrm{PhCH}_{2} \mathrm{O}$ ), 4.76 (d, J=7.8 Hz, 1H, H-1"), 4.65 (d, $\left.J=10.7 \mathrm{~Hz}, 1 \mathrm{H}, \mathrm{PhCH}_{2} \mathrm{O}\right), 4.24(\mathrm{ddd}, J=9.8 \mathrm{~Hz}, J=4.8 \mathrm{~Hz}, J=2.2 \mathrm{~Hz}, 1 \mathrm{H}), 4.10$ (dd, $J$ $=10.2 \mathrm{~Hz}, J=8.9 \mathrm{~Hz}, 1 \mathrm{H}), 3.79(\mathrm{dd}, J=8.9 \mathrm{~Hz}, J=8.8 \mathrm{~Hz}, 1 \mathrm{H}), 3.64(\mathrm{dd}, J=9.7 \mathrm{~Hz}, J$ $=9.2 \mathrm{~Hz}, 1 \mathrm{H}), 3.4-3.5(\mathrm{~m}, 7 \mathrm{H}), 3.3-3.4(\mathrm{~m}, 2 \mathrm{H}), 3.28(\mathrm{dd}, J=10.3 \mathrm{~Hz}, J=3.8 \mathrm{~Hz}$, $1 \mathrm{H}), 3.08(\mathrm{dd}, J=9.6 \mathrm{~Hz}, J=9.6 \mathrm{~Hz}, 1 \mathrm{H}), 2.36(\mathrm{ddd}, J=12.9 \mathrm{~Hz}, J=3.8 \mathrm{~Hz}, J=3.8 \mathrm{~Hz}$, $\left.1 \mathrm{H}, \mathrm{H}-2_{\mathrm{eq}}\right), 1.47$ (s, 9H), 1.38 (d, $\left.J=6.1 \mathrm{~Hz}, 3 \mathrm{H}, \mathrm{H}-6 ”\right), 1.36\left(\mathrm{~m}, 1 \mathrm{H}, \mathrm{H}-2_{\mathrm{ax}}\right) ;{ }^{13} \mathrm{C}$ NMR (100 MHz, $\left.\mathrm{CDCl}_{3}\right) \delta 157.1$ (s), 138.0 (s), 137.9 (s), 133.8 (s), 130.4 (s), 128.7 (s), 128.3 (s), 128.1 (s), 127.9 (s), 104.1 (s), 96.4 (s), 86.1 (s), 81.3 (s), 80.0 (s), 78.9 (s), 77.4 (s), 76.0 (s), 75.7 (s), 75.3 (s), 75.2 (s), 75.1 (s), 71.6 (s), 71.0 (s), 67.6 (s), 63.2 (s), 60.4 (s), 51.4 (s), 50.4 (s), 33.3 (s), 28.5 (s), 18.3 (s); MALDI Calcd for $\mathrm{C}_{37} \mathrm{H}_{49} \mathrm{~N}_{13} \mathrm{O}_{11} \mathrm{Na}$ $\left([\mathrm{M}+\mathrm{Na}]^{+}\right) \mathrm{m} / \mathrm{e} 874.3567$; measure $\mathrm{m} / \mathrm{e} 874.3525$.

\section{5-O-(4-Azido-4,6-dideoxy- $\beta$-D-glucopyranosyl)-3',4'-di-O-benzyl-1- $N$-[(S)-4-}

(benzyloxycarbonylamino)-2-hydroxybutanoyl]-3,2',6'-triazidoneamine (18). Please refer to the procedure for synthesis of compound $15 .{ }^{1} \mathrm{H} \mathrm{NMR}\left(400 \mathrm{MHz}, \mathrm{CDCl}_{3}\right) \delta 7.3-$ $7.4(\mathrm{~m}, 15 \mathrm{H}), 6.03\left(\mathrm{~d}, J=3.7 \mathrm{~Hz}, 1 \mathrm{H}, \mathrm{H}-1^{\prime}\right), 5.0-5.1\left(\mathrm{~m}, 3 \mathrm{H}, \mathrm{PhCH}_{2} \mathrm{O}\right), 4.91(\mathrm{~d}, J=10.2$ $\left.\mathrm{Hz}, 1 \mathrm{H}, \mathrm{PhCH}_{2} \mathrm{O}\right), 4.86\left(\mathrm{~d}, J=10.8 \mathrm{~Hz}, 1 \mathrm{H}, \mathrm{PhCH}_{2} \mathrm{O}\right), 4.71$ (d, $J=7.7 \mathrm{~Hz}, 1 \mathrm{H}, \mathrm{H}-1$ "), $4.62\left(\mathrm{~d}, J=11.4 \mathrm{~Hz}, 1 \mathrm{H}, \mathrm{PhCH}_{2} \mathrm{O}\right), 4.25(\mathrm{~m}, 1 \mathrm{H}), 4.10(\mathrm{dd}, J=9.2 \mathrm{~Hz}, J=9.9 \mathrm{~Hz}, 1 \mathrm{H})$, $3.76(\mathrm{dd}, J=8.6 \mathrm{~Hz}, J=9.0 \mathrm{~Hz}, 1 \mathrm{H}), 3.1-3.6(\mathrm{~m}, 13 \mathrm{H}), 3.0-3.1(\mathrm{~m}, 2 \mathrm{H}), 2.29(\mathrm{~m}, 1 \mathrm{H}$, $\left.\mathrm{H}-2_{\mathrm{eq}}\right), 1.7-1.9(\mathrm{~m}, 2 \mathrm{H}), 1.44\left(\mathrm{~m}, 1 \mathrm{H}, \mathrm{H}-2_{\mathrm{ax}}\right), 1.39$ (d, $\left.J=5.3 \mathrm{~Hz}, 3 \mathrm{H}, \mathrm{H}-6 "\right) ;{ }^{13} \mathrm{C} \mathrm{NMR}$ 
(100 MHz, $\left.\mathrm{CDCl}_{3}\right) \delta 174.9$ (s), 158.0 (s), 138.0 (s), 137.9 (s), 136.5 (s), 128.8 (s), 128.7 (s), 128.5 (s), 128.4 (s), 128.2 (s), 128.1 (s), 127.9 (s), 104.4 (s), 96.3 (s), 86.3 (s), 80.0 (s), 78.9 (s), 76.1 (s), 75.7 (s), 75.5 (s), 75.3 (s), 75.1 (s), 71.5 (s), 70.9 (s), 67.5 (s), 67.3 (s, 2 carbons), 63.2 (s), 60.4 (s), 51.4 (s), 48.7 (s), 46.2 (s), 37.3 (s), 34.9 (s), 32.7 (s), 18.3 (s); MALDI Calcd for $\mathrm{C}_{44} \mathrm{H}_{54} \mathrm{~N}_{14} \mathrm{O}_{13} \mathrm{Na}\left([\mathrm{M}+\mathrm{Na}]^{+}\right) \mathrm{m} / \mathrm{e}$ 1009.3887; measure m/e 1009.3838 .

\section{5-O-(4-Amino-4,6-dideoxy- $\beta$-D-glucopyranosyl)-1- $N$-[(S)-4-amino-2-}

hydroxybutanoyl]neamine (JT005). Please refer to refernce $8 .{ }^{1} \mathrm{H}$ NMR $(400 \mathrm{MHz}$, $\mathrm{D}_{2} \mathrm{O}$ ) (chloride salt) $\delta 5.86\left(\mathrm{~d}, J=4.0 \mathrm{~Hz}, 1 \mathrm{H}, \mathrm{H}-1^{\prime}\right), 5.18(\mathrm{~d}, J=8.1 \mathrm{~Hz}, 1 \mathrm{H}, \mathrm{H}-1 "), 4.31$ (dd, $J=8.0 \mathrm{~Hz}, J=4.0 \mathrm{~Hz}, 1 \mathrm{H}), 3.8-4.1(\mathrm{~m}, 8 \mathrm{H}), 3.68(\mathrm{dd}, J=9.6 \mathrm{~Hz}, J=9.8 \mathrm{~Hz}, 1 \mathrm{H})$, $3.5-3.6$ (m, 5H), 3.34 (dd, $J=13.7 \mathrm{~Hz}, J=5.8 \mathrm{~Hz}, 1 \mathrm{H}), 3.1-3.2(\mathrm{~m}, 2 \mathrm{H}), 2.28$ (ddd, $J$ $=12.7 \mathrm{~Hz}, J=4.3 \mathrm{~Hz}, J=4.3 \mathrm{~Hz}, 1 \mathrm{H}, \mathrm{H}-2_{\mathrm{eq}}$ ), $2.16(\mathrm{~m}, 1 \mathrm{H}), 1.97(\mathrm{~m}, 1 \mathrm{H}), 1.83$ (ddd, $J=$ $\left.12.7 \mathrm{~Hz}, J=12.5 \mathrm{~Hz}, J=12.5 \mathrm{~Hz}, 1 \mathrm{H}, \mathrm{H}-2_{\mathrm{ax}}\right), 1.42$ (d, $\left.J=6.3 \mathrm{~Hz}, 3 \mathrm{H}, \mathrm{H}-6 "\right) ;{ }^{13} \mathrm{C} \mathrm{NMR}$ (100 MHz, $\mathrm{D}_{2} \mathrm{O}$ ) (chloride salt) $\delta 175.9$ (s), 102.7 (s), $96.4(\mathrm{~s}), 81.5$ (s), 76.7 (s), 74.5 (s), 73.8 (s), 72.0 (s), 70.7 (s), 69.86 (s, 2 carbons), 69.80 (s), 68.4 (s), 56.9 (s), 53.7(s), 49.4 (s), $48.8(\mathrm{~s}), 40.2(\mathrm{~s}), 36.9(\mathrm{~s}), 31.1(\mathrm{~s}), 30.2(\mathrm{~s}), 17.0(\mathrm{~s})$. 


\section{Anisotropic Effect from Molecular Modeling}

Molecular modeling of 6-O-Benzoyl-3',4'-di-O-benzyl-1,3,2',6'-tetraazidoneamine (4) (top) and 5,6Di-O-benzoyl-3',4'-di-O-benzyl-1,3,2',6'-tetraazidoneamine (5) (bottom). Except for H-6', H-2', H1, and H-3, all the hydrogen atoms are omitted for clarity. The H-2' proton in compound 4 is about $4 \AA$ away from the carbonyl group at the O-5 position, which could have little but important anisotropic effect.
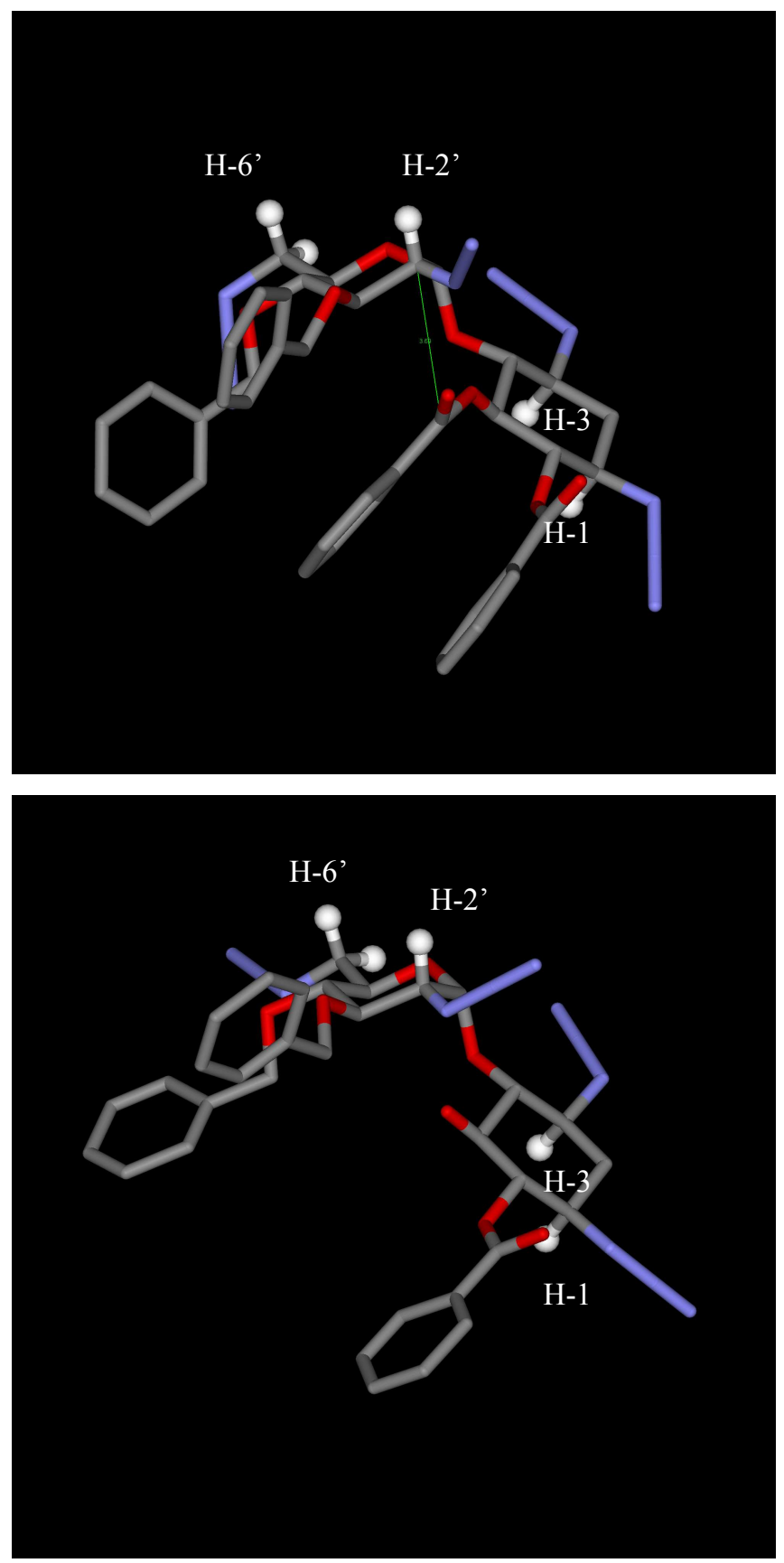

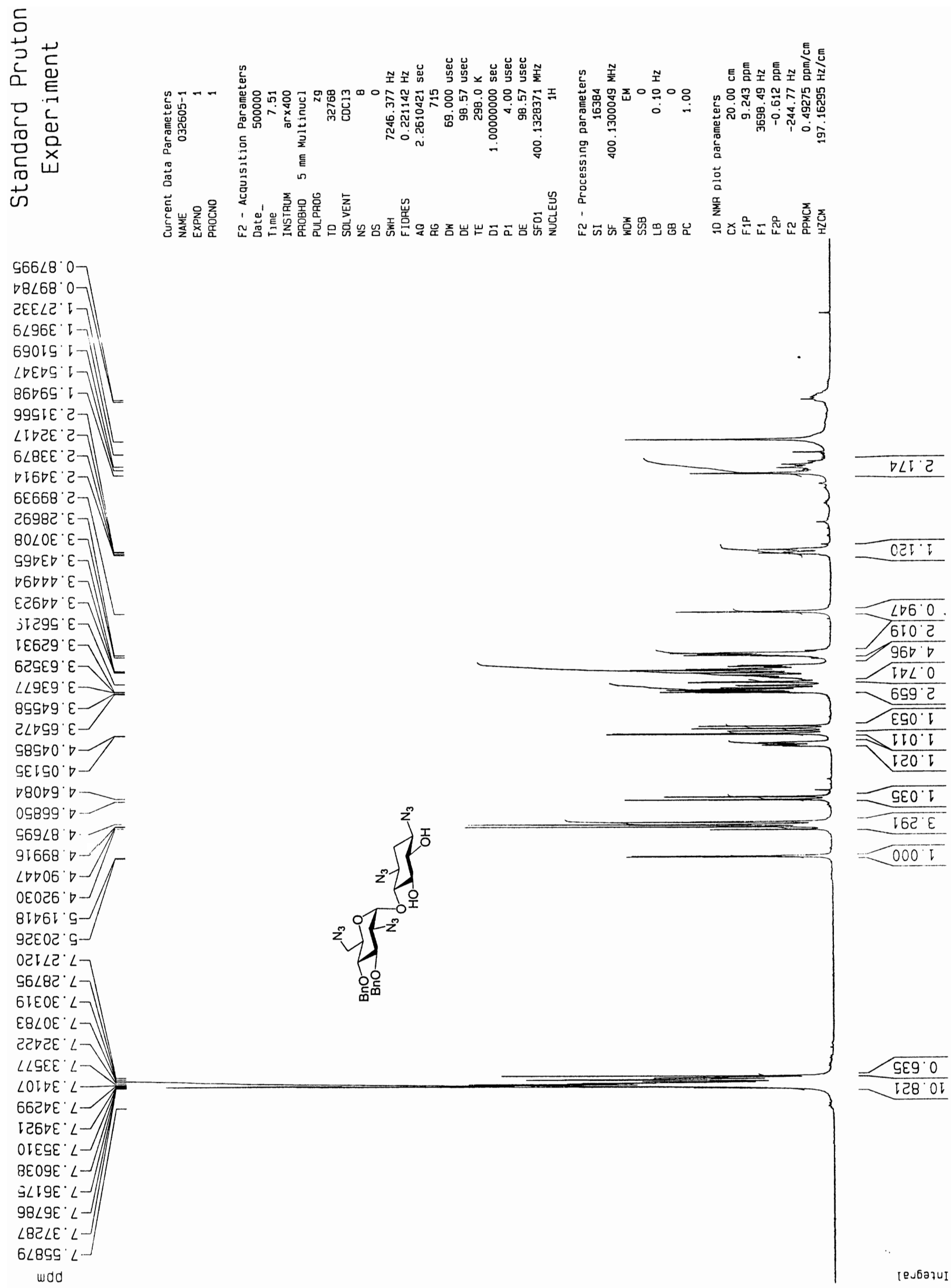


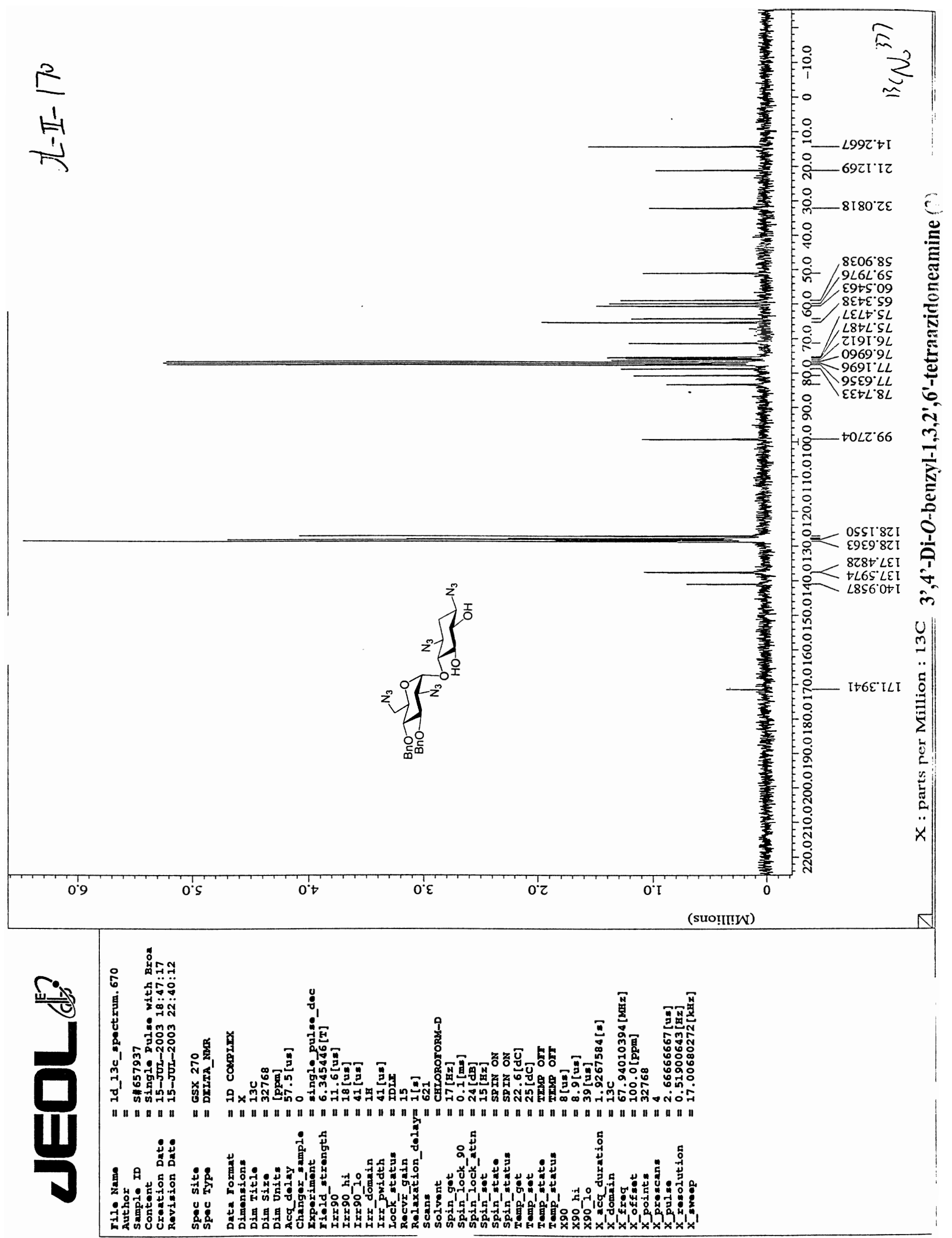




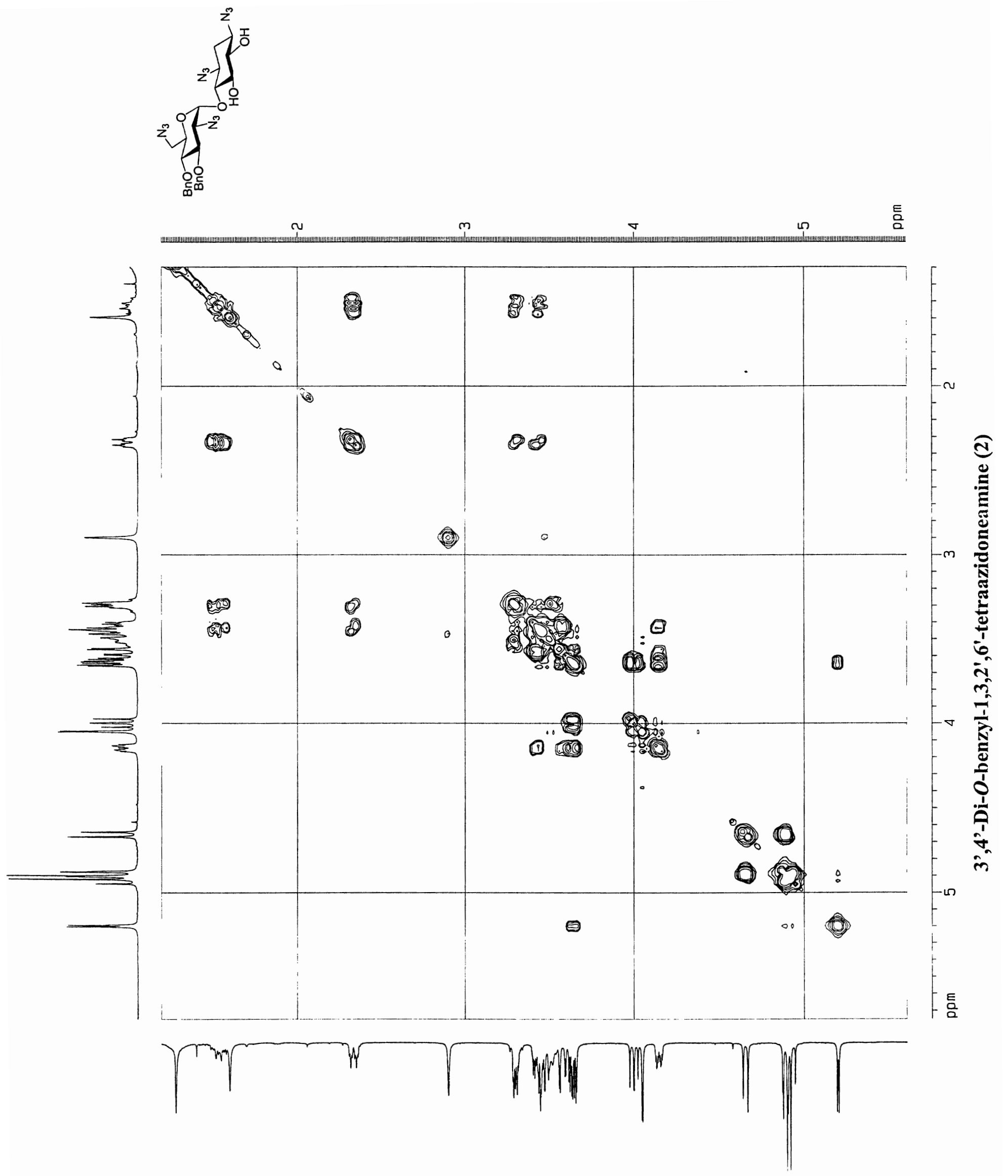




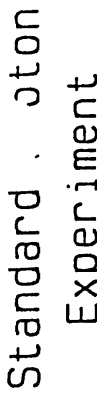

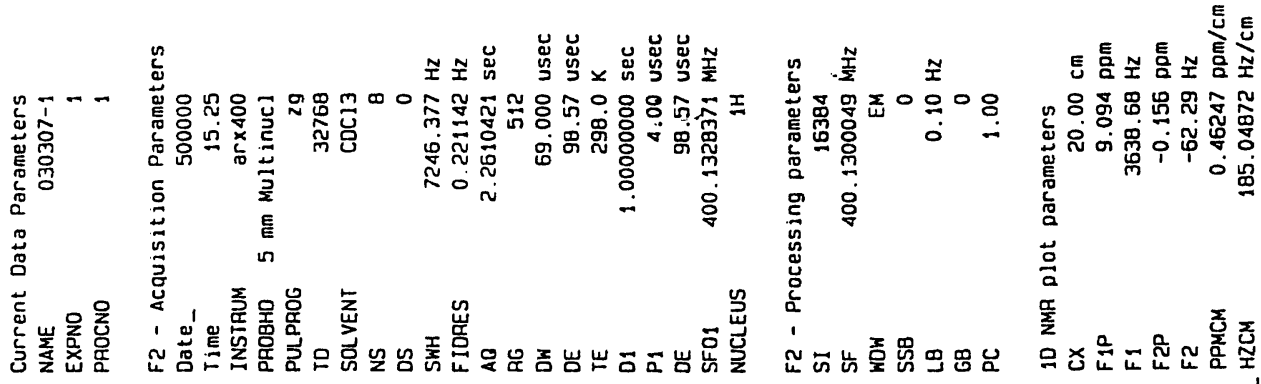

$\angle 6 I \angle C^{\prime} \cdot$

$885 \angle 2 \cdot[-$

$\left.\angle 0089^{\circ} \cdot\right]$

90119.1

$\triangle \nabla D \Delta 9^{\circ} 17$

ODSSO 5

$\triangle 9860^{\circ} \mathrm{C}$

Sट90I. 5

66510.5] E

डD०टा. 57

OS9D० 27

SIESE' $\varepsilon>1 V$

$\left.985 \angle \varepsilon^{\circ} E\right]$

टटLटD ${ }^{\circ}-7$

$9 ट \angle E D \cdot E-$

$\angle D C E S^{\prime} \varepsilon-$

$99 \angle 6 S^{\circ} \varepsilon>$

$\mathrm{S}^{\prime} 6 \mathrm{~S}^{\prime} \varepsilon>$

ताटटक. $\varepsilon$

५9L29. $\varepsilon$

LLISG $E$

$05896^{\circ} \varepsilon$

$86029 \cdot$

I LCS9.

SIILB $\square=$

I $\triangle 8 \angle B^{\circ}$

$\angle 6668^{\circ} \cdot$

$0 \angle 9 D 6^{\circ} \circ$

टडा $\angle 6^{\circ} \circ$

टा8टा. $\mathrm{s}$

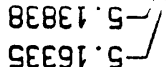

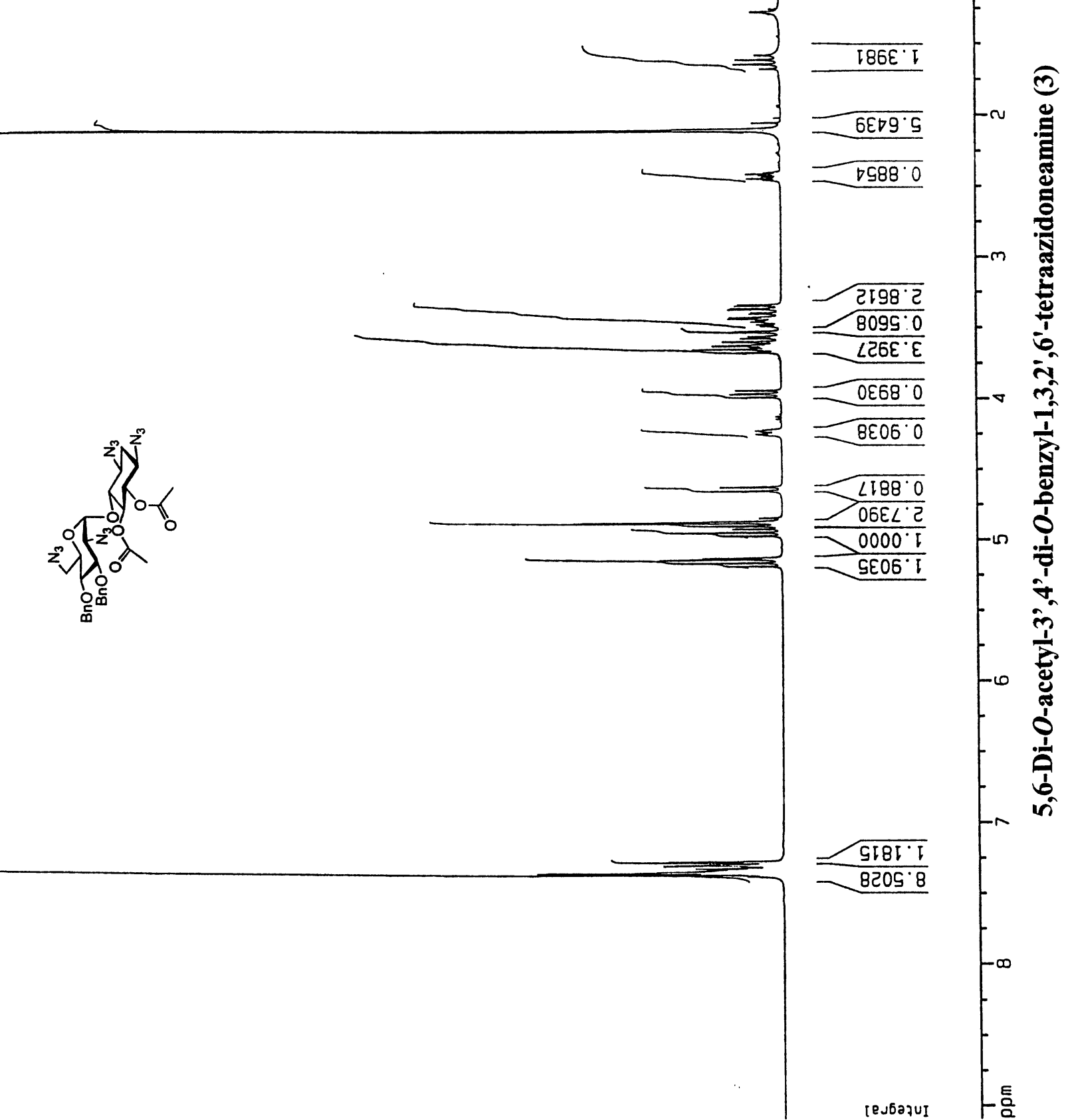

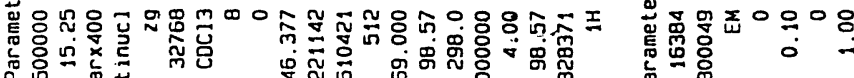

$\stackrel{5}{3}$

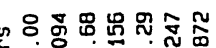

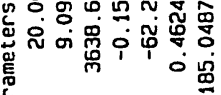

$0 \angle 0<2 \circ]$

SISLC $\angle]$

$988<2 \cdot \angle \neg$

टा०6ट ' $\angle \neg \backslash$

$\begin{array}{ll}26862 \cdot \angle> \\ 86 \angle I E & \angle>\end{array}$

$\angle 692 E^{\prime} \angle$

GOOEE' $L$

G9DEE ' $L$

$6 \angle D D E^{\circ} \angle$

$\angle \nabla O D E$ ' $\angle$

ES6จE $L$

$\nabla \angle S E^{\circ} \angle$

S909E $\angle-$

Elg9E $<-J$ 


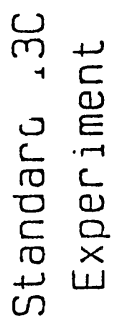

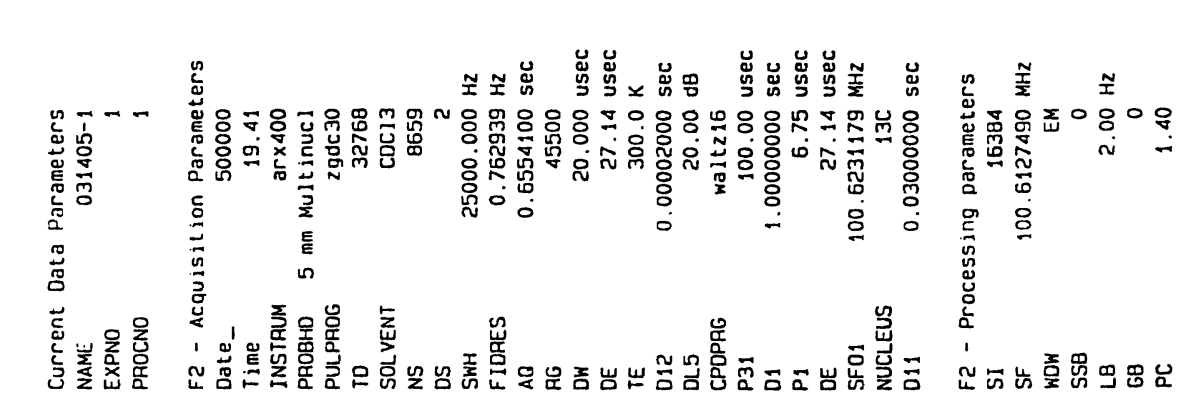

튼

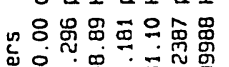

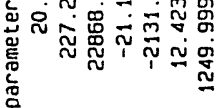

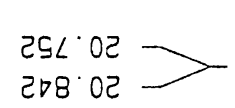

$\triangle D O .2 E-$ 600. Is $\triangle 26^{\circ} \angle S$ $\angle I 6.89$ $600^{\circ} \varepsilon 9$ 619. $I L$ $969^{\circ} \varepsilon L$ $\nabla ट E^{\prime} \nabla L$ $6 S D^{\circ} S L$ $\angle 69^{\circ} S \angle$ $868 \cdot 9 L$ 9Iट $\angle L$ DES $\angle L$ SOL' $B L$ $206^{\circ} 8 L$ $889^{\circ} 6 L$ GDE 66 810.821 281.851 995. 851 $8 I L \cdot 8 ट T$ E9L 82 I O9L $\angle E I$ $\subseteq B \angle \angle E I$

$589^{\circ} 691$

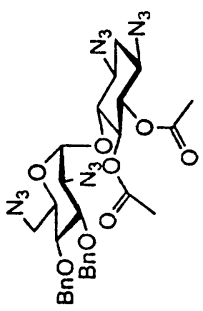
$210.0<1$ 


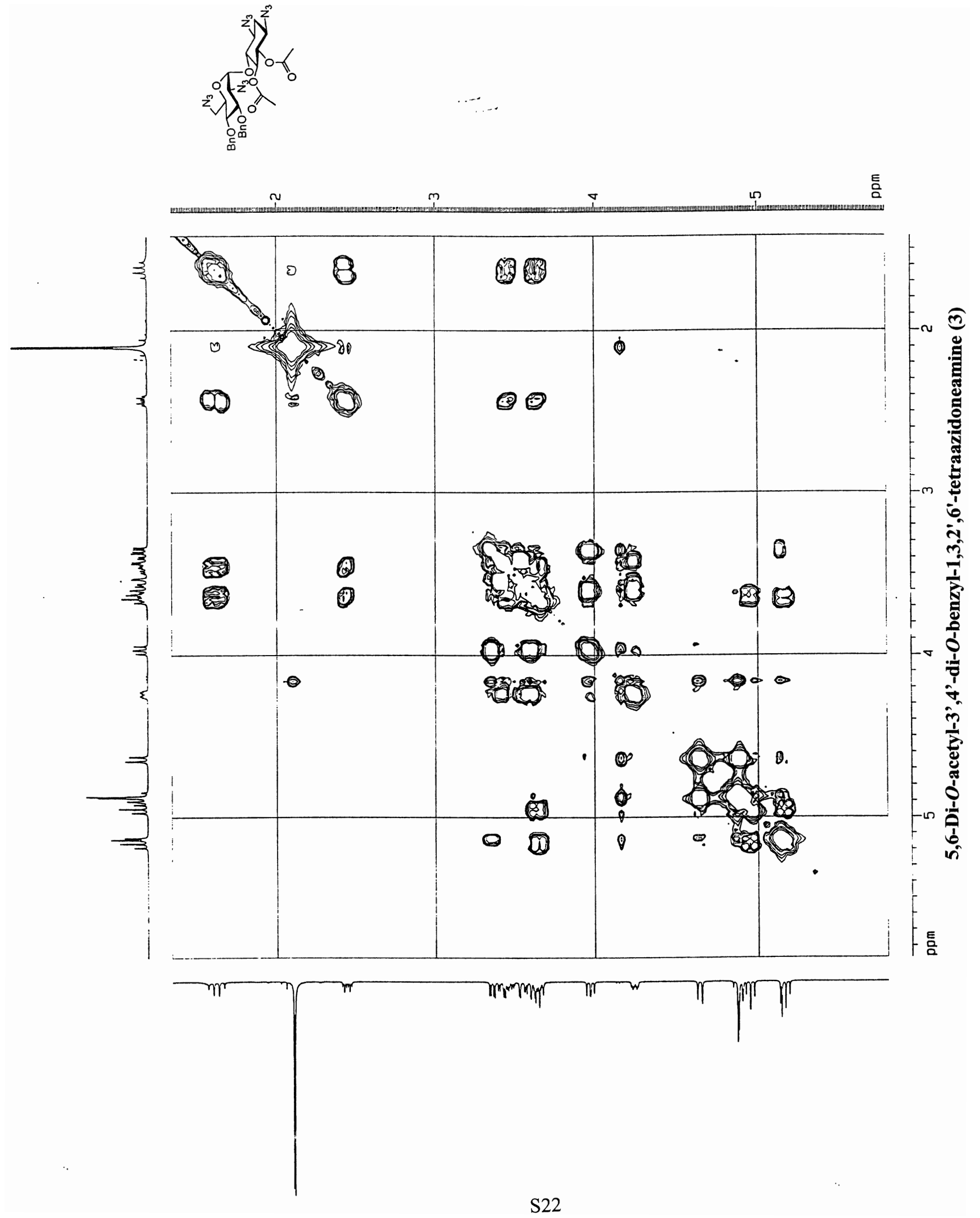


15

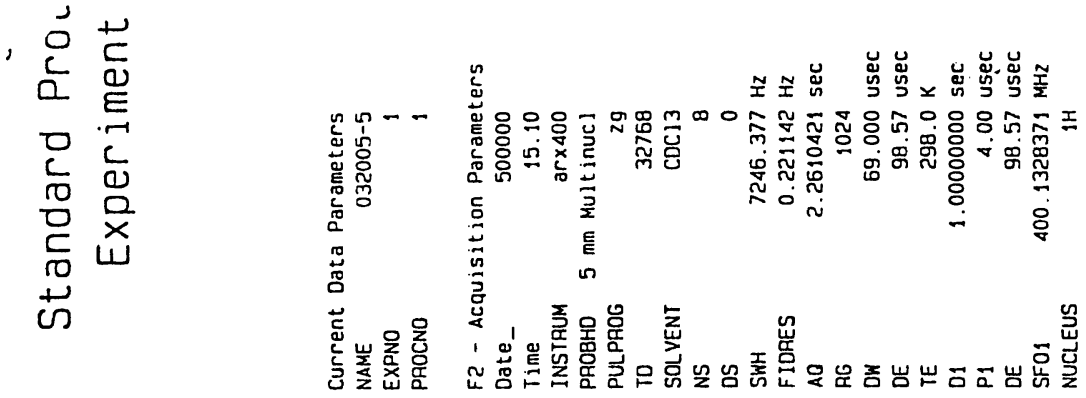

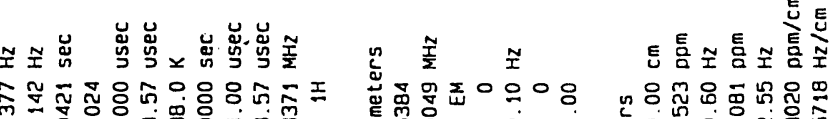

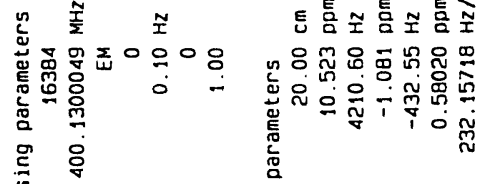

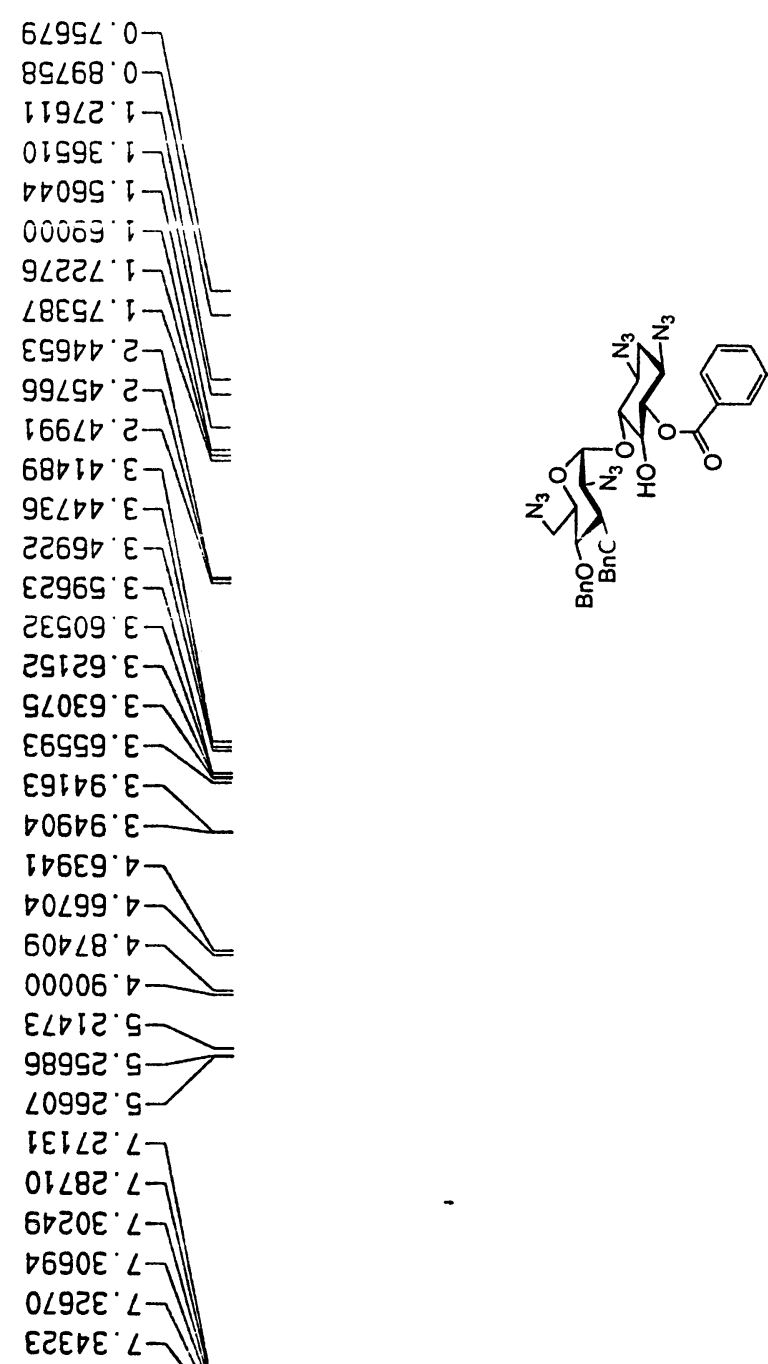

OIISE $\angle>$

SEDSE $\angle$

бEम9E ' $\angle$

9889E $\angle$

โ8290 $\angle-$

$28180^{\circ} \angle-$

6SLOS $\angle-$

$\angle \angle \angle O 9^{\circ} \angle-$

$\angle S 6600^{\circ} 8-$

8OEOL 8 -

$0 \angle 0 Z L^{\circ} 8-$

OEEट Г 8

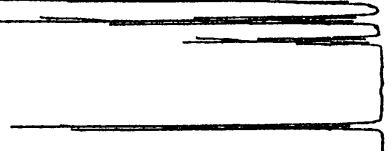

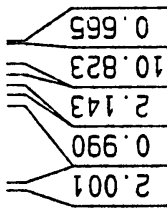

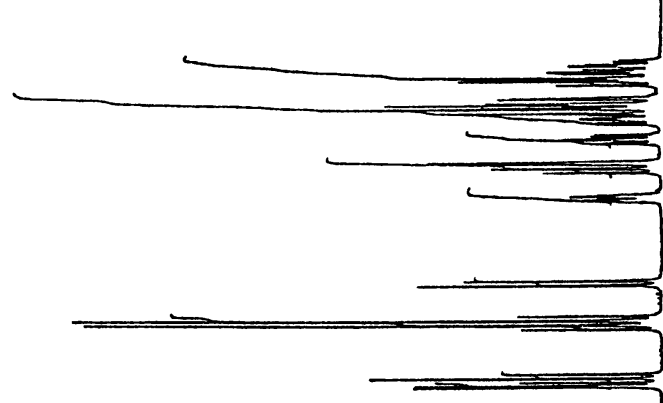

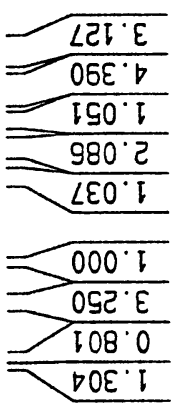

$=$

E16.0 

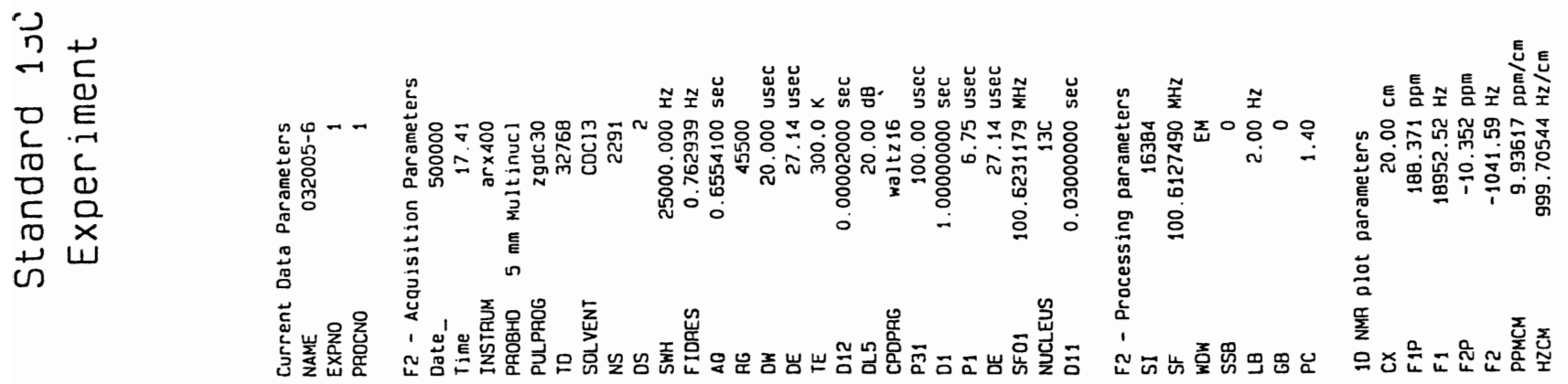

S૦७. टЕ -

69I. IS

$6 \nabla 5.89$

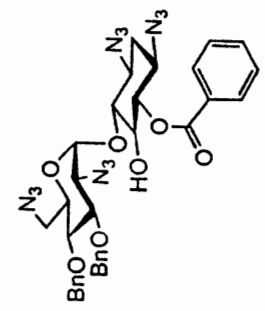

$180^{\circ} 89$
189.62

$\left.\begin{array}{l}029 \cdot I L \\ S \angle L\end{array}\right]$

SLL DL

$860^{\circ} \mathrm{g} L$

$\nabla 06^{\circ} S L$

ट68. $9 L$

$602 . \angle L$

925. $\angle L$

Е૬८. 8

St0. 18

$88 \varepsilon \cdot \triangleright 8$

$69<\cdot 66$

โ20.82

$815 \cdot 85[7]$

$\left.\begin{array}{l}169.851 \\ \text { OIE. } 851\end{array}\right]$

6टL 821

$\triangle 6 L \cdot 821$

ED० 6टा-

$9 \angle I$ OEI

IOL EEโ -

O6. ' $\angle E I-$

$92 \angle \angle E I-$

$890^{\circ} 991$

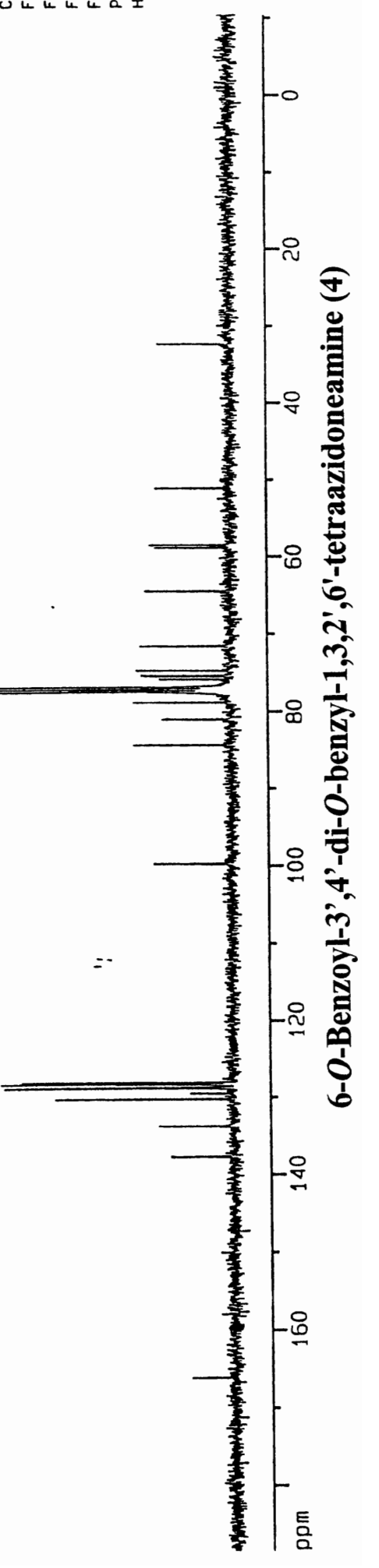



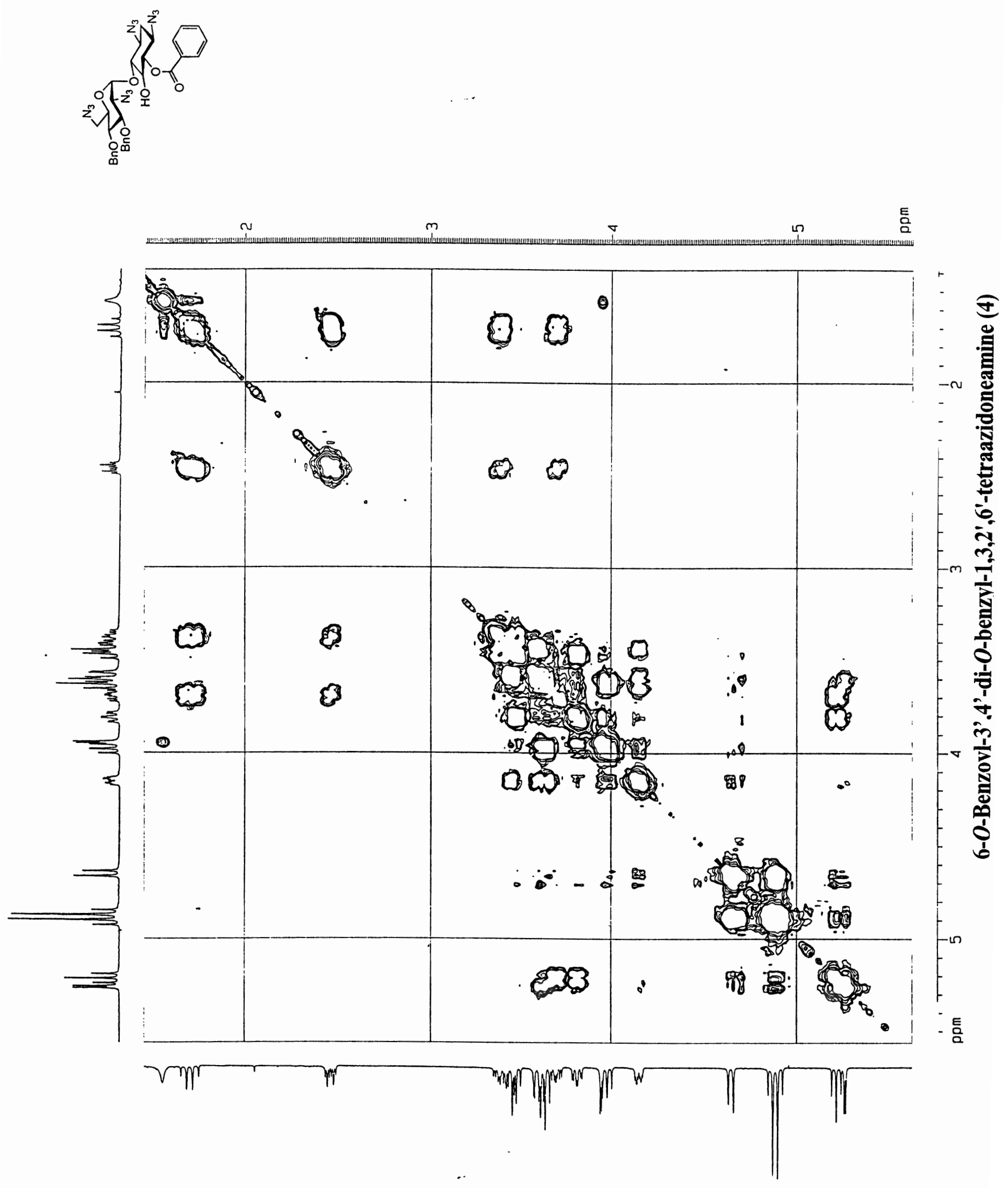
1 등
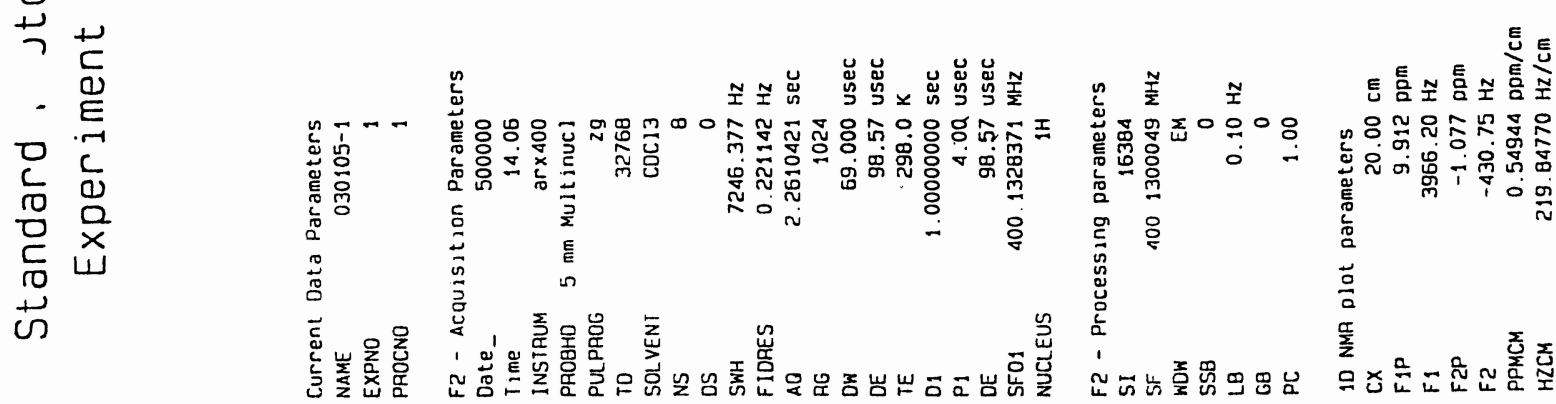

$\triangle 2 \angle \angle \theta^{\circ}$

$68 \angle 68^{\circ} 0$

SS9L2

S9०9E. $\tau-$

Bโ86L $[7] \quad=$

BLOEB' $[7$

$\left.\varepsilon \nabla 298^{\circ} \cdot \neg\right)$

69LL[']

E6St5. $57 \mid$

$0 \supset 6 \angle 9^{\circ} \mathrm{C}-$

$\varepsilon 8 \circ 9 \Gamma^{\circ} \varepsilon-$

टा00ट. $\left.E-{ }^{2}\right]=$

टOBOS $\mathrm{C}$

OSZSS $\varepsilon$

$\operatorname{Sgt} \angle \theta^{\circ} \varepsilon-[$

ST088 $\varepsilon-$

$\left.\nabla 668^{\circ} \varepsilon\right]$

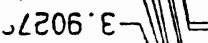

SจS06 $\varepsilon$

ESD89. $\square$

O०टा9.

$E \angle \nabla L L^{\circ}$

OEEG $L^{\circ}$

$\left[\angle \nabla E 8^{\circ} \circ\right.$

$89298^{\circ}$

OEOLI. $\mathrm{S}$

$\angle \angle 6 Z T \cdot S$

O6LEE.

6I9E9. 9

$621 \angle C^{\circ} \angle$

ट69Lट $L$

$98082 \cdot \angle$

$88882 \cdot \angle 7$

DIE6C $\angle 7$

SEIIE $\angle>$

टL8टE

$\angle$ LCEE

DOGEE

$\triangle B \angle D E^{\circ} \angle$

9EDSE $\angle$

8IBSE '

Eจ99E :

66LLE

:O98E

$20006^{\circ} \mathrm{L}$

$18126^{\circ} \mathrm{L}$

SI $6 E 0.8$

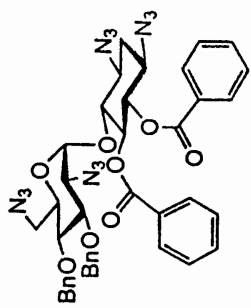

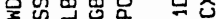




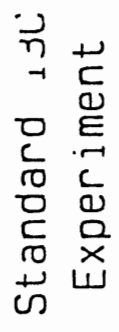

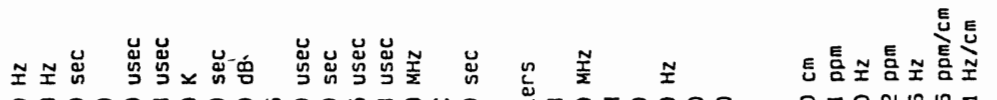

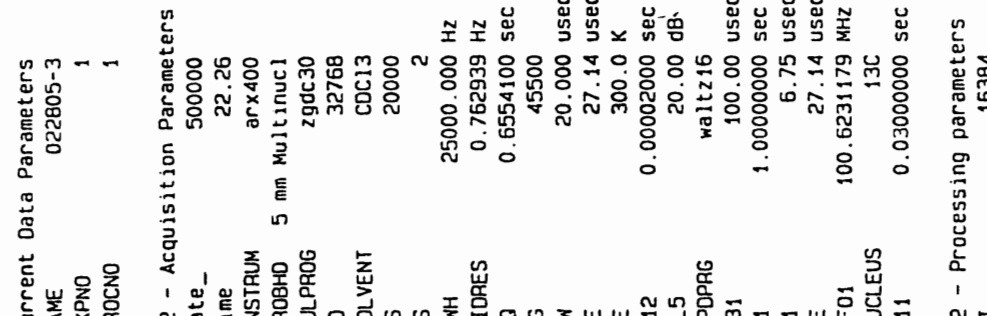

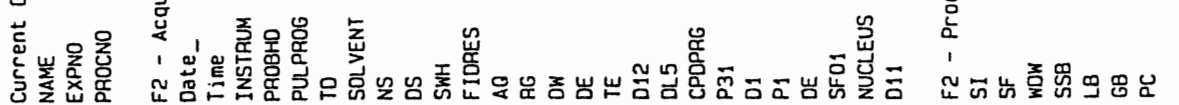

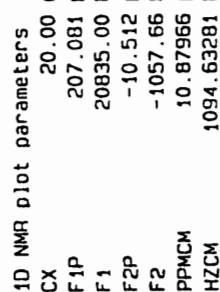
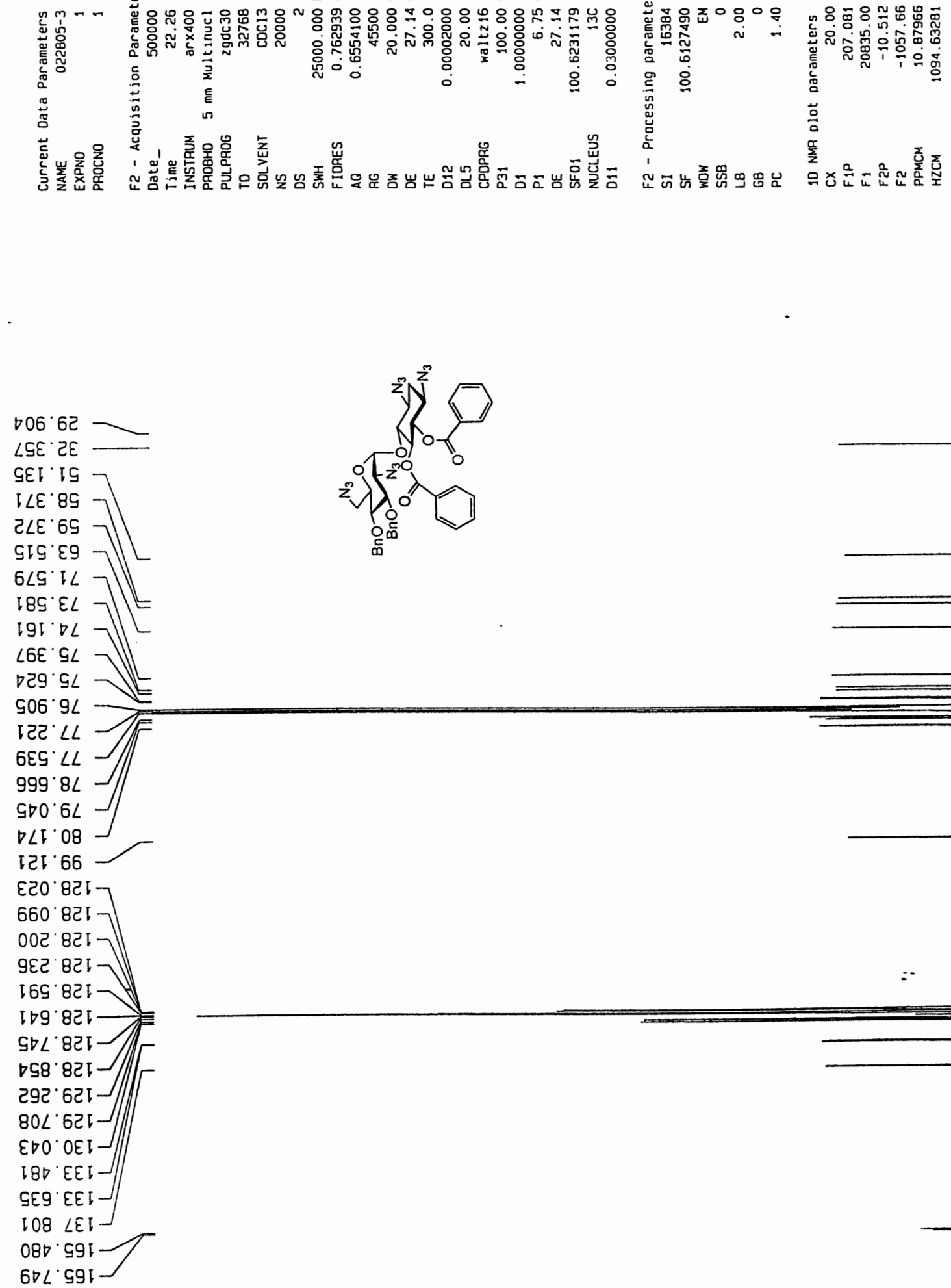


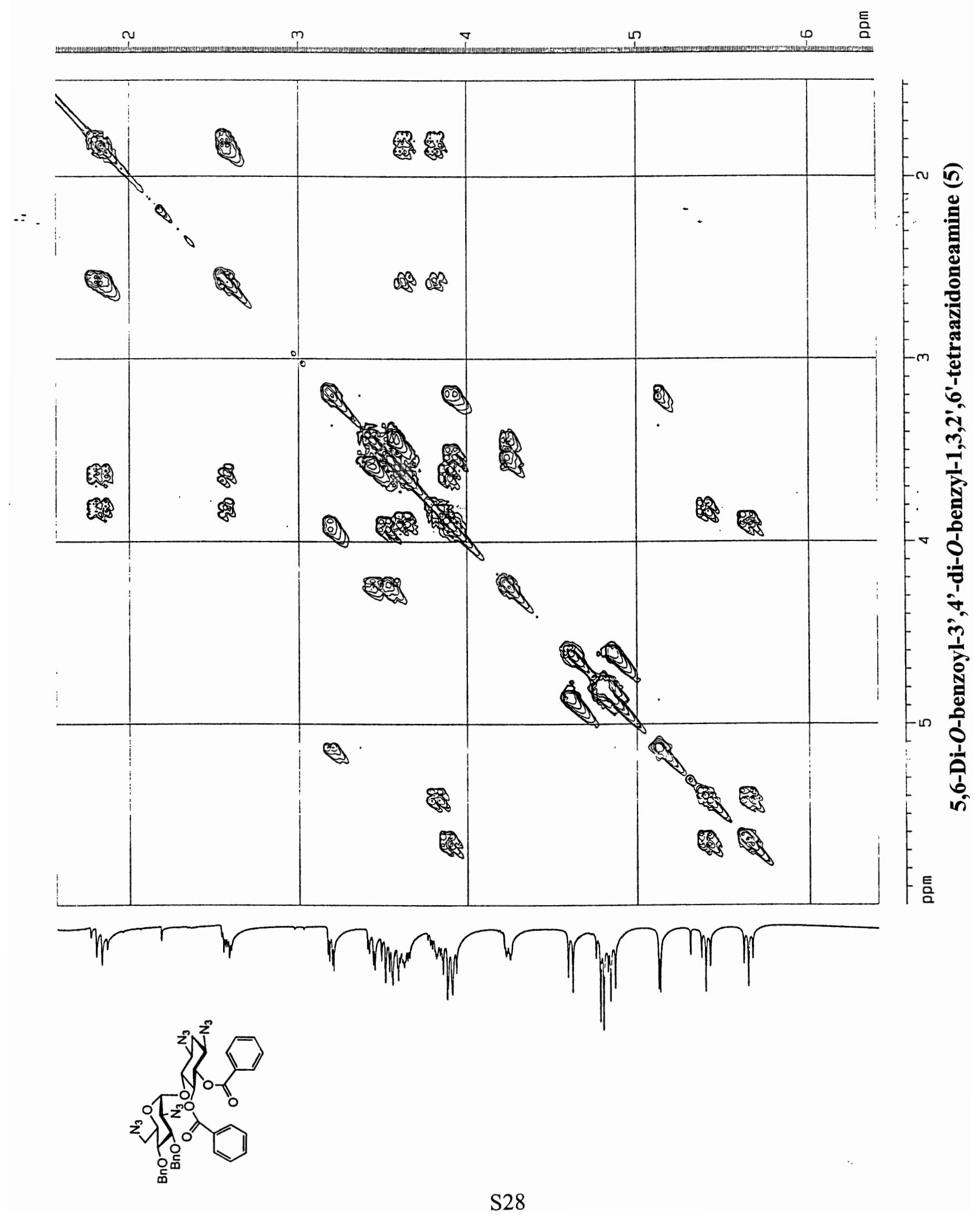


, ᄃ

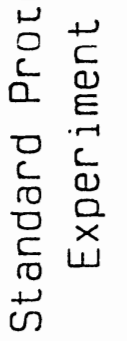

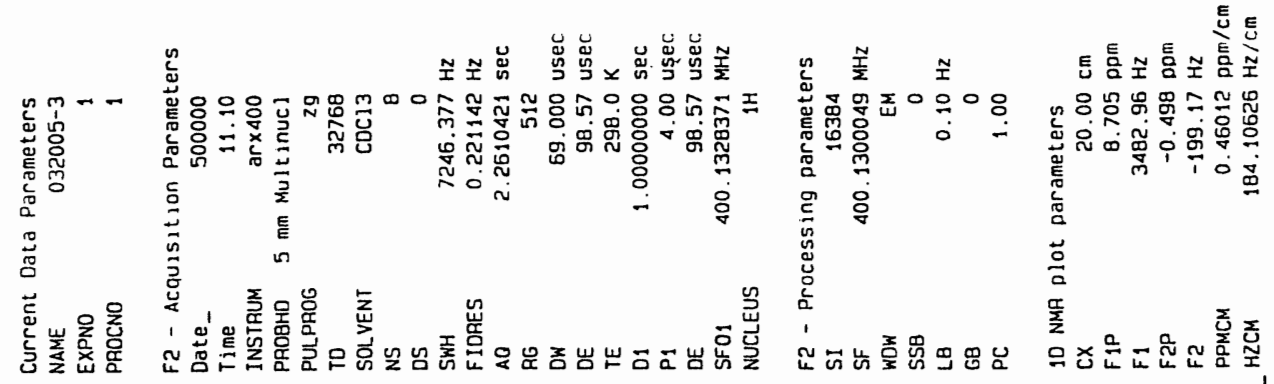

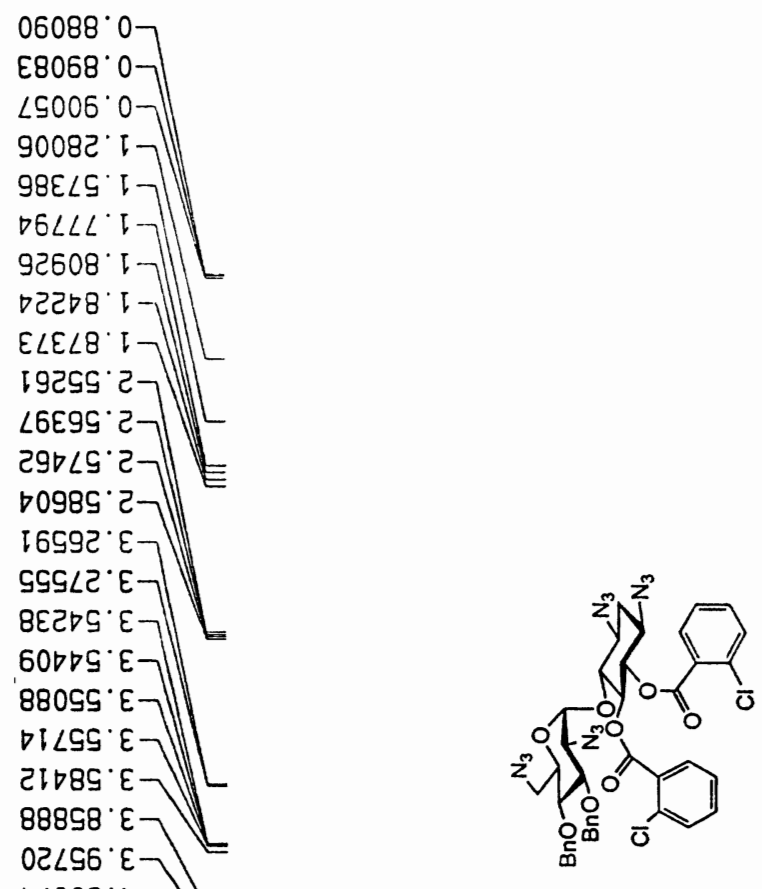

$\nabla \angle 109^{\circ} \square$

$89629 \cdot \square]$

[ $\left.\angle O E 8^{\circ} \cdot \nabla\right]$

6S6E8' $\square$

$\angle O D S 8^{\circ}$

$\angle 8188^{\circ}$

EStII

G6Eटा $\longrightarrow$

$\varepsilon 2000 \cdot 9$

OSGED. $\longrightarrow$

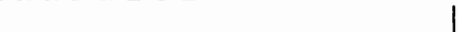

ESII9.9

[60LC $\angle-$

टा $\left.980^{\circ} \angle\right]$

$80062 \cdot L$

DDIIE $L 7$

809LE $L>$

LСटटE' $\angle 7$

EटLCE'L

EIOEE

Q6IEE

BLODE $\angle$

EटBSE ' $L$

टाटбE $\angle-$

$[\angle D G E \cdot L]$

टDEED $\angle$
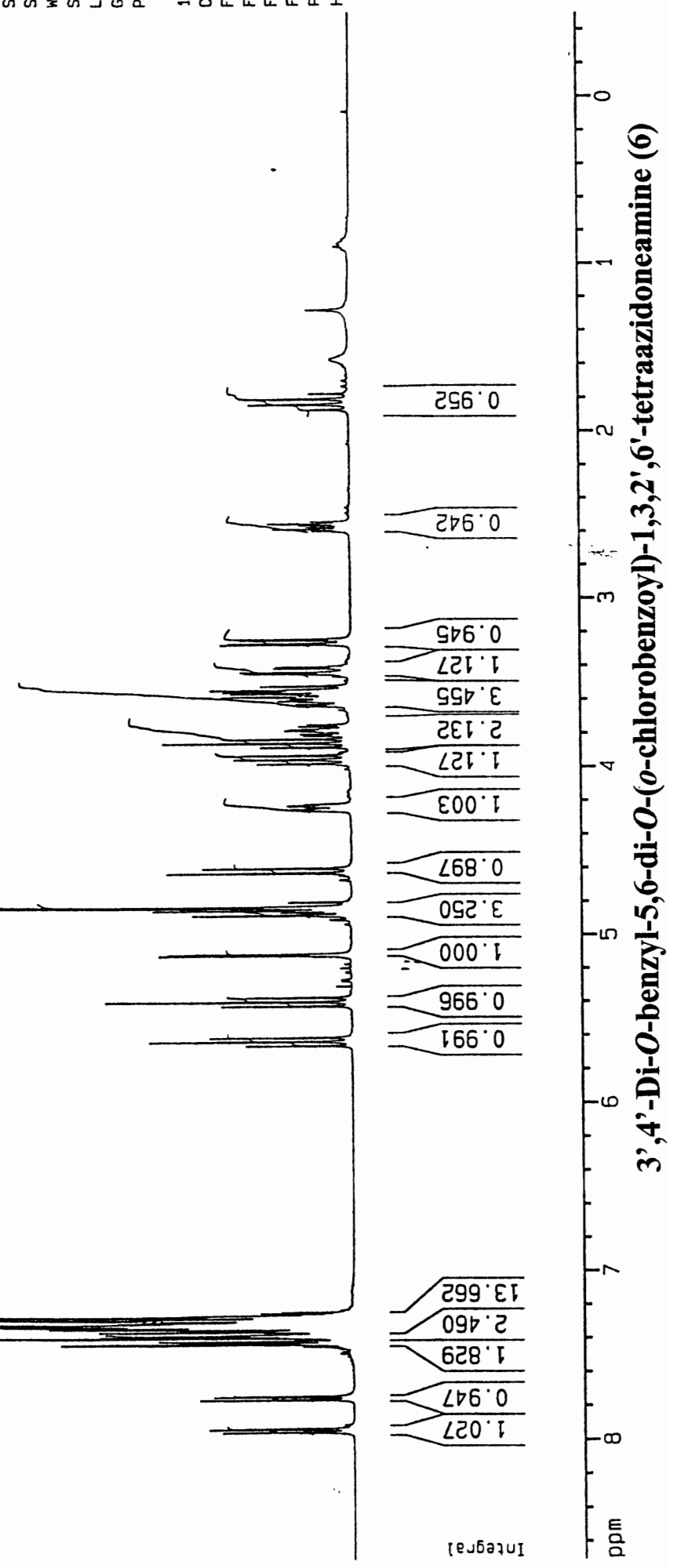


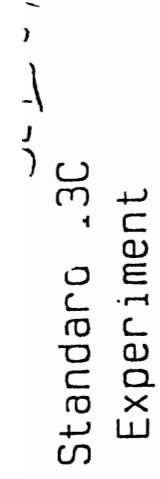
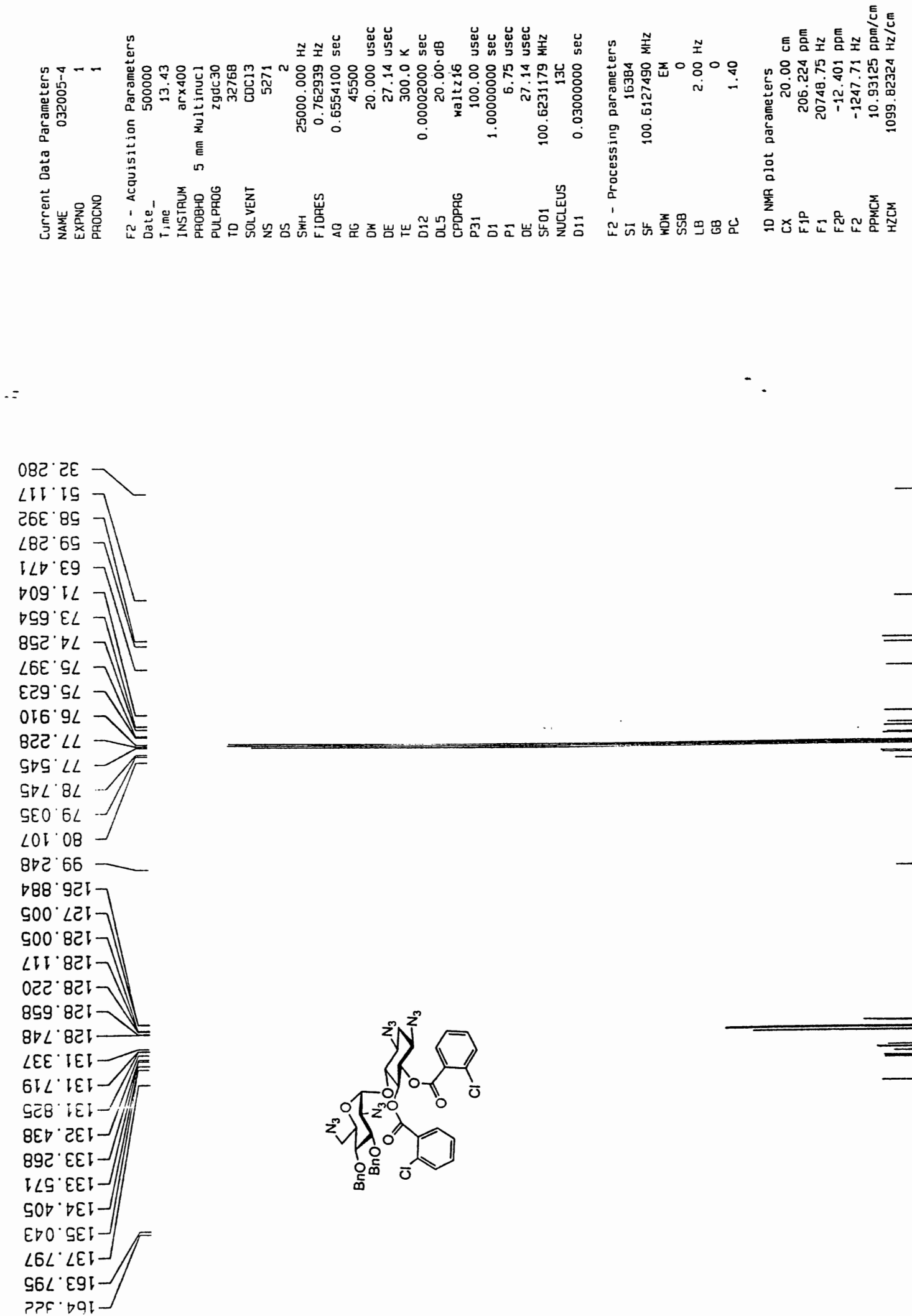


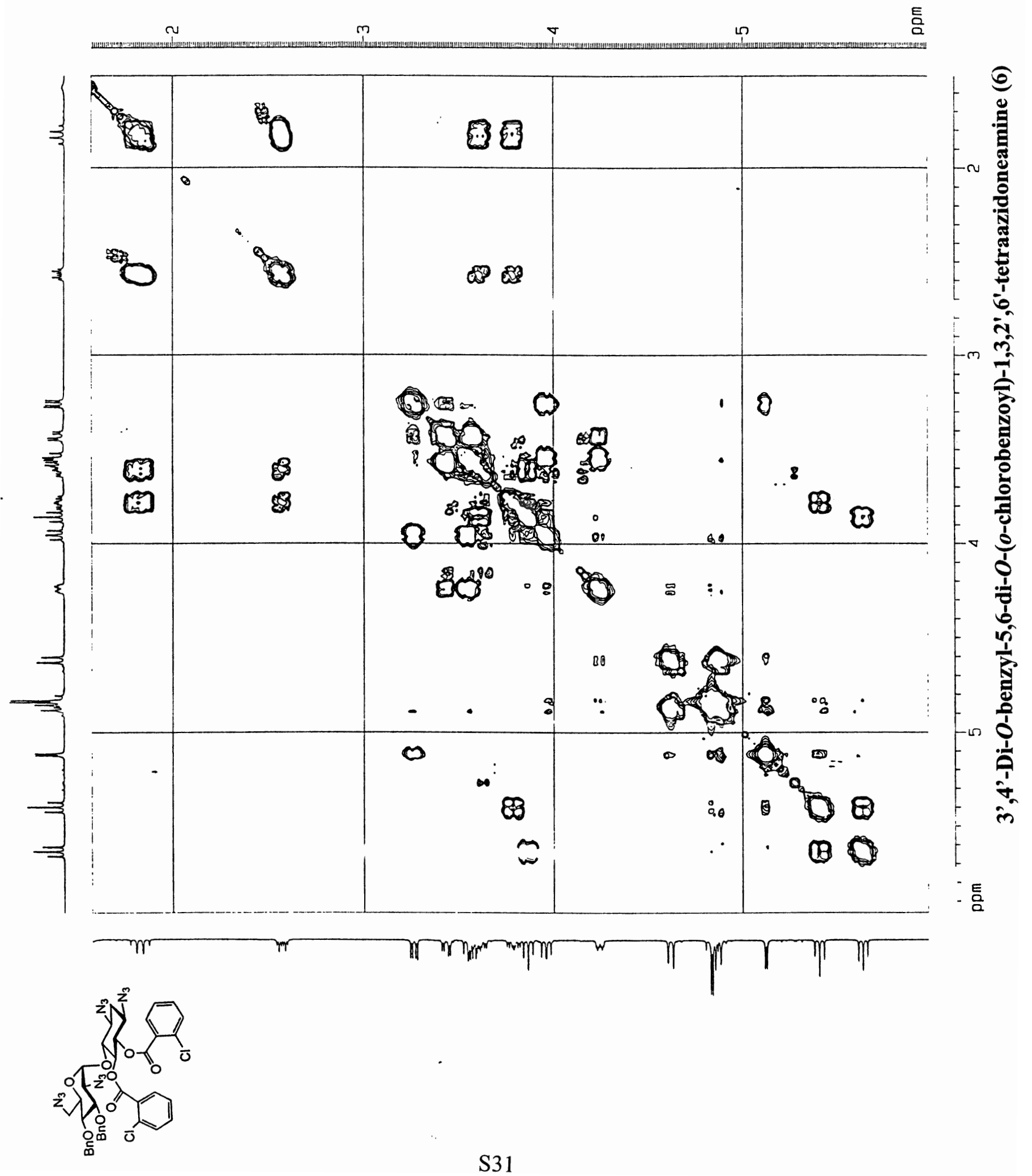



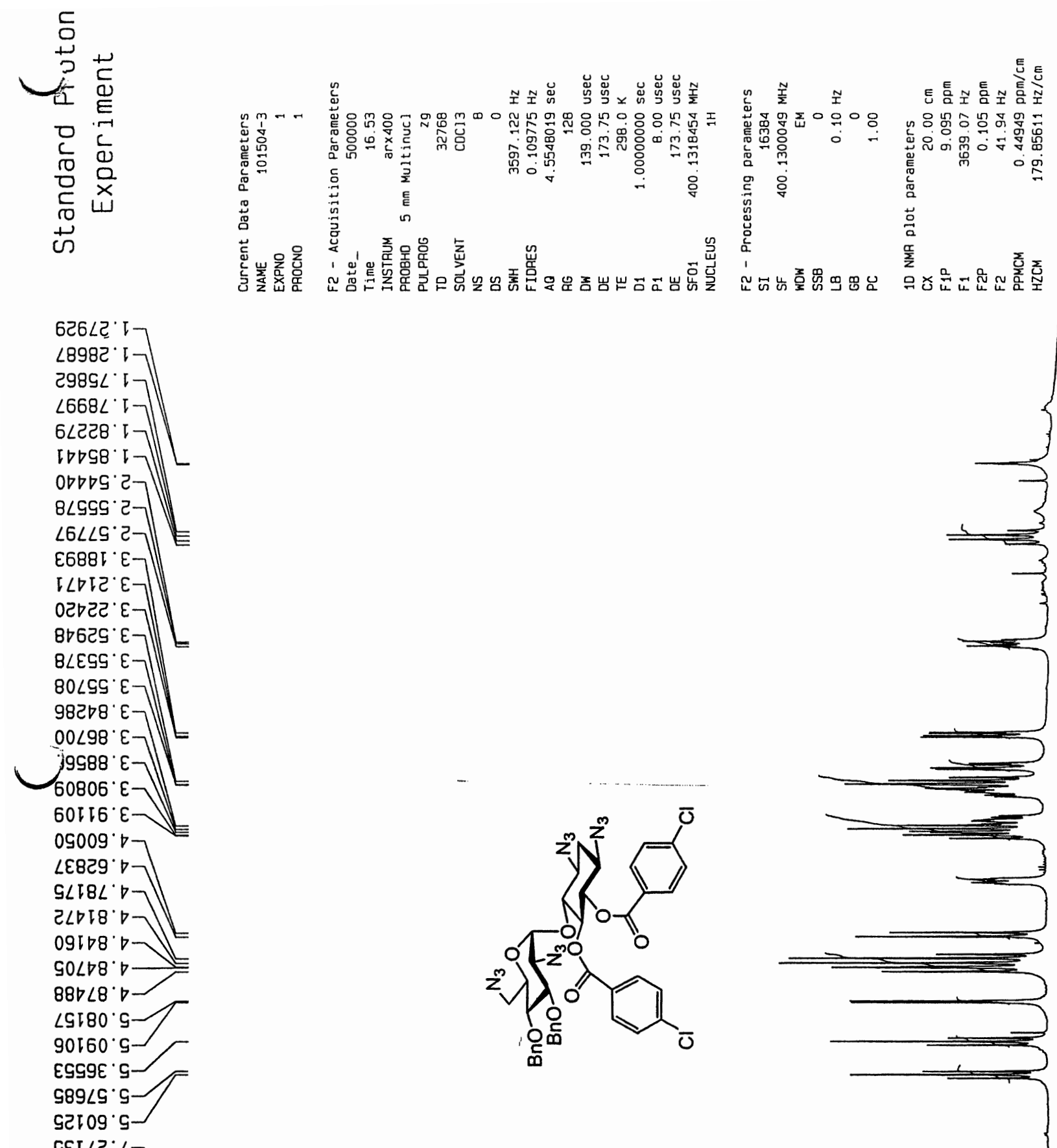


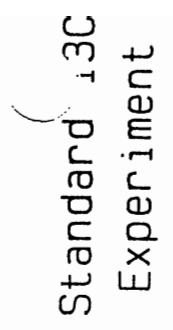
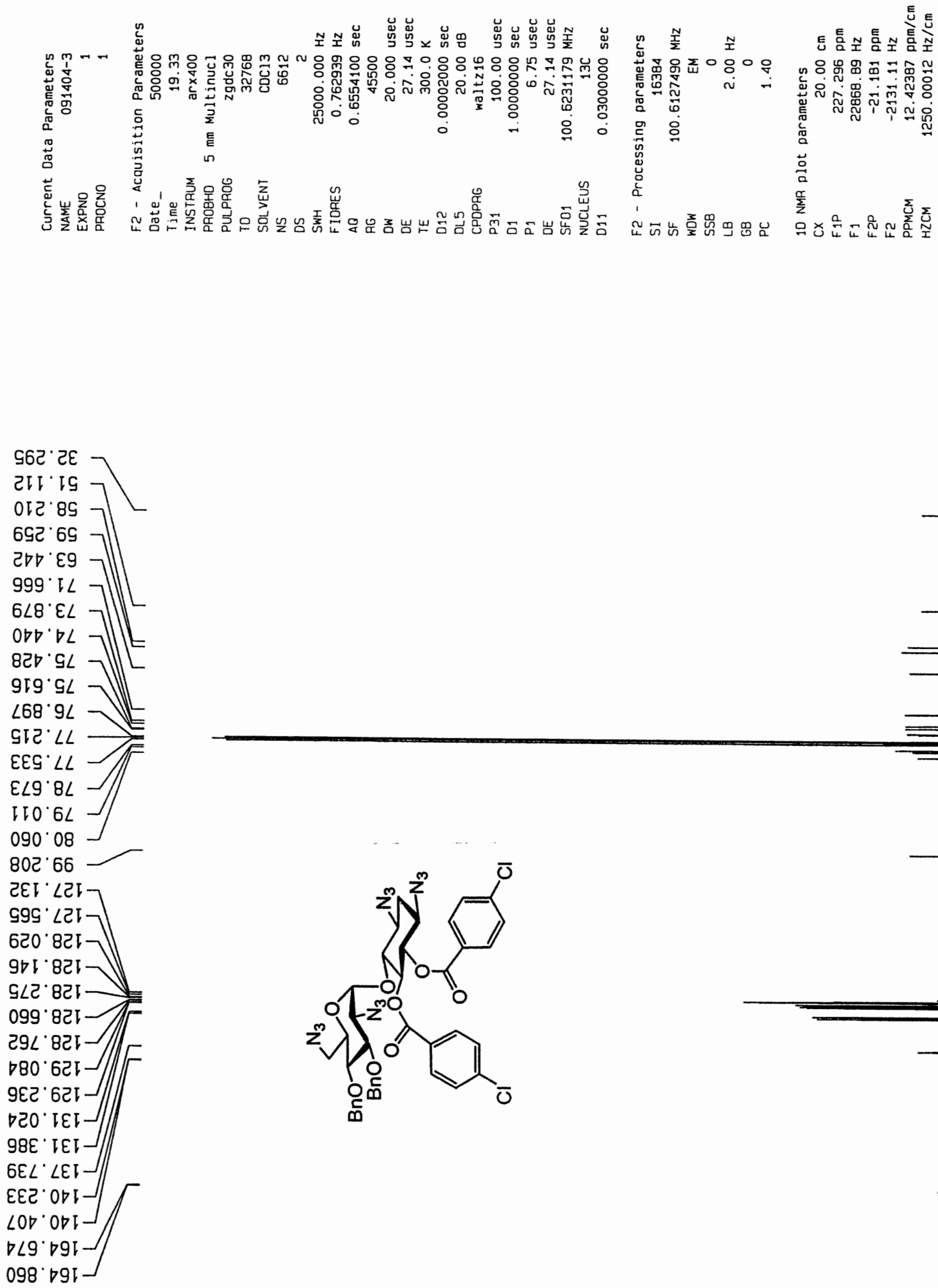

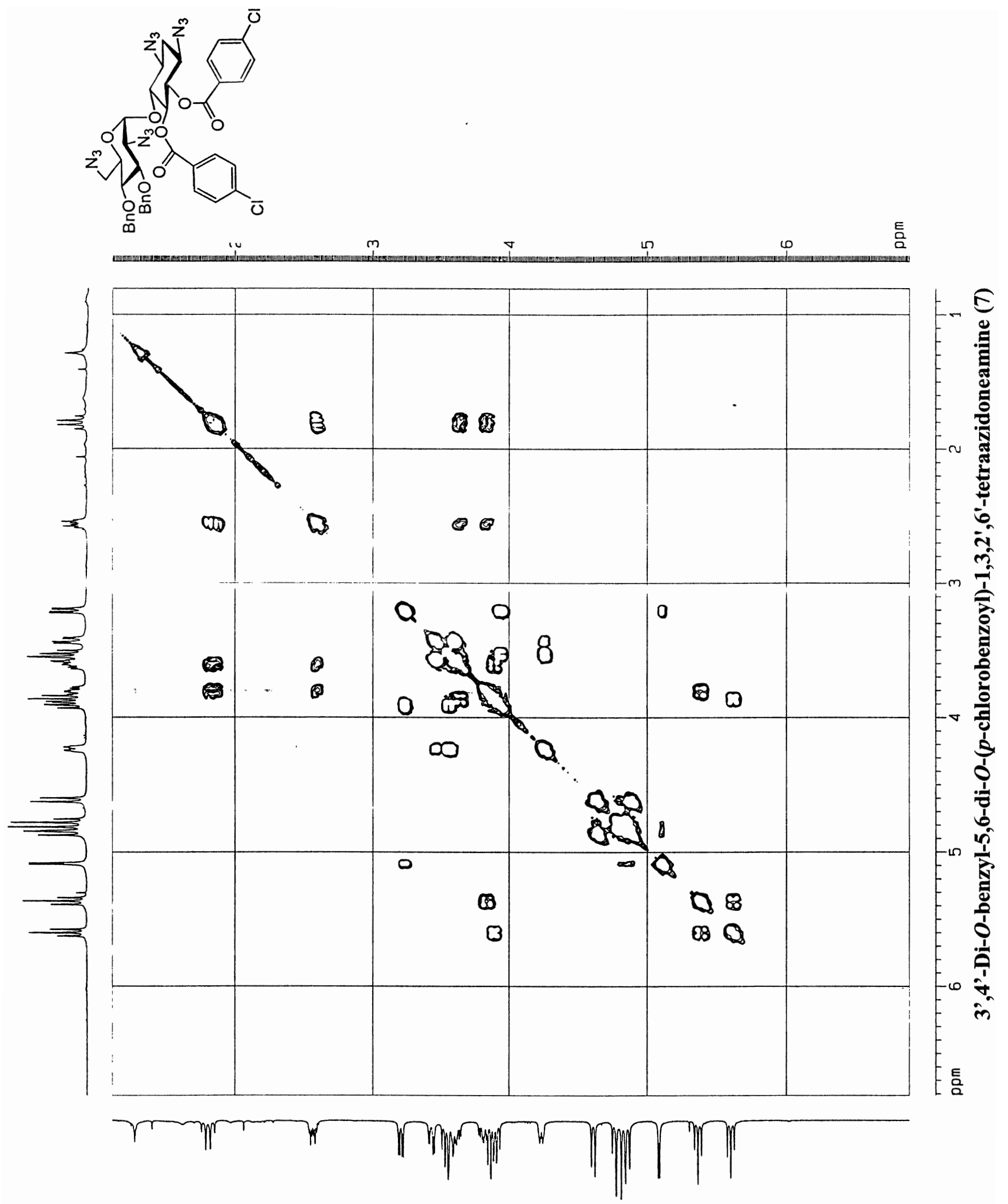


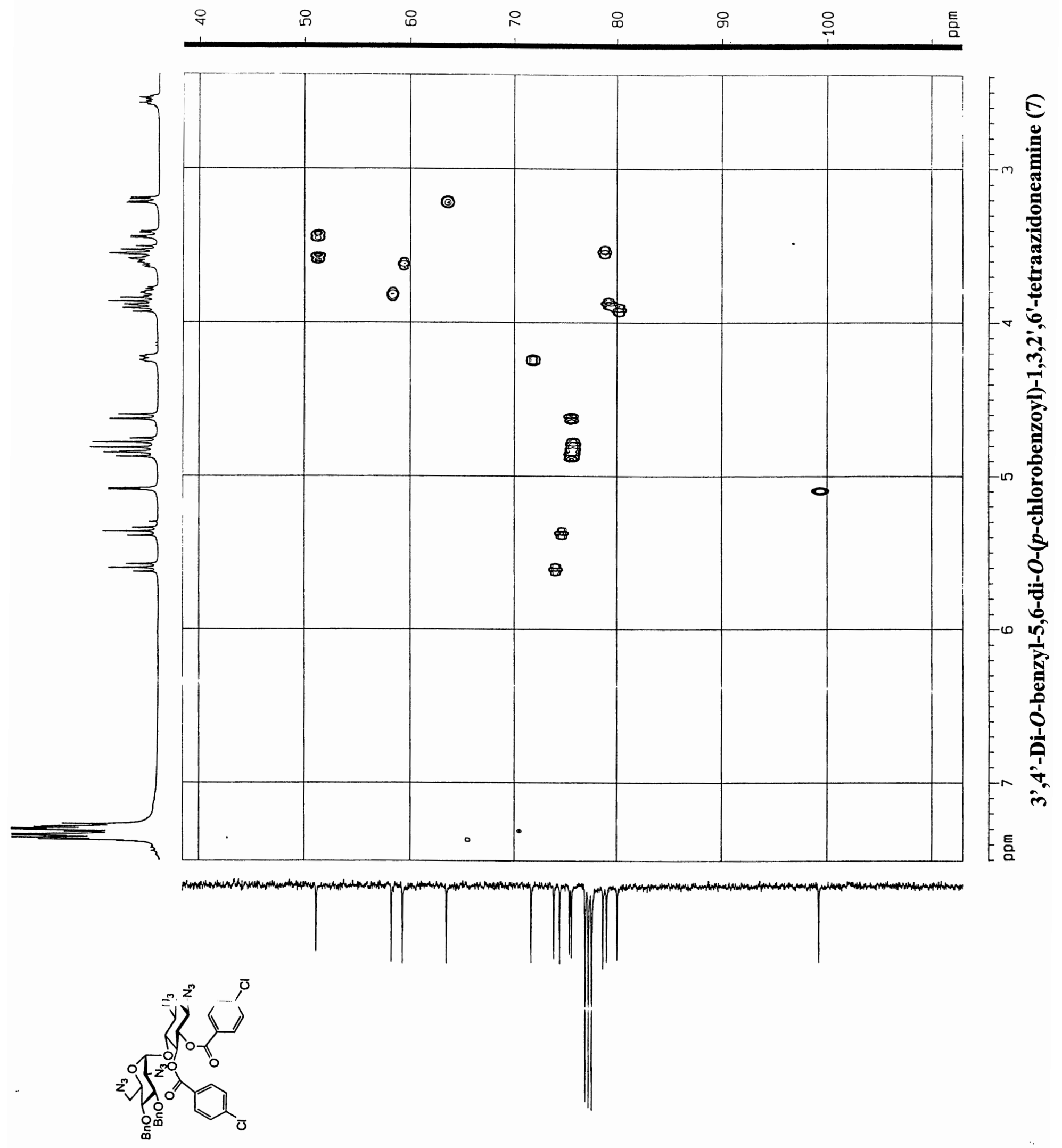


돈

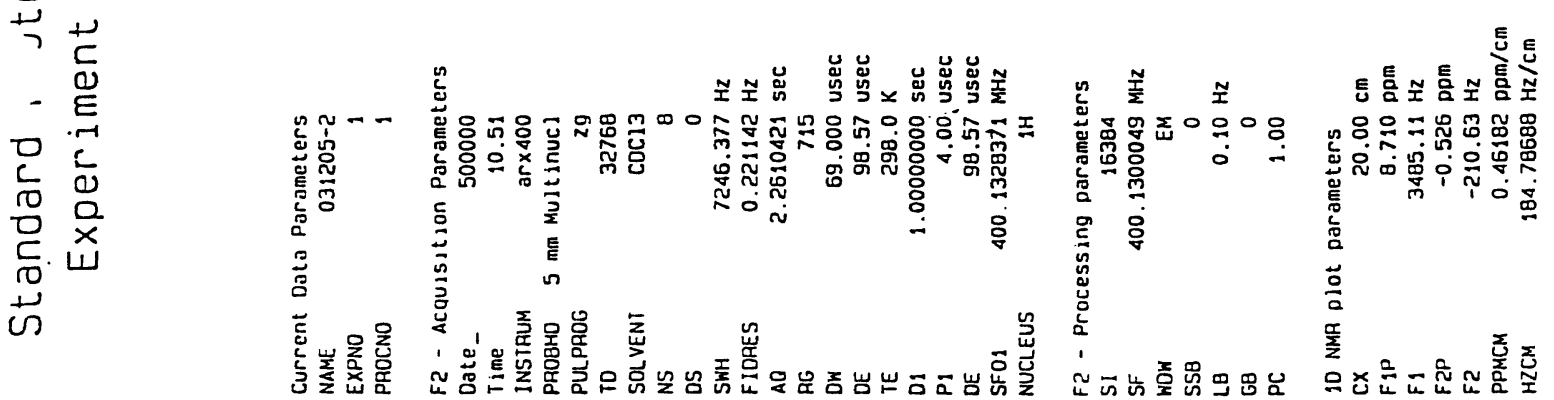

$9 \angle 968^{\circ} 0$

BIELC' I

SOLED' $[-$

OISB० I- =

$\angle 6 \angle I S \cdot T-11 !$

$8 \angle 6051-1$

EULES 1-

SEESO $2 \ldots$

$09 \angle 60^{\circ} 2$ - 管

टा160.57)

OSEZS. 57

จOSDE.E]

SBDSE' $E$ ]

$\triangle 8\left[\angle E^{\prime} E\right]$

टBOBE' $E$ -

गडटा ह $E 7$

JDED'E $\varepsilon$

$\left.0 \angle L 2 S^{\circ} \varepsilon\right]$

ऽटE9S $\varepsilon$

SIgLS $\varepsilon$

$80885^{\circ} \varepsilon$

I2009. $\varepsilon-$

IOLIT.

OIDSE E-

ESBL6. $E$

9०6०टन

$9 \angle 029$.

ट58०9 $\circ$

LESGL口 DIIf

डाI5B. ${ }^{\circ}-1$

$018 \angle B^{\circ} \nabla-$

$\triangle \triangle 000^{\circ} \circ$

25201. $\mathrm{a}$

DIDIT

DL66I'S-

S6E2ट 9

ट8692 $L$

टSILC $L>$

CDLLC. $\angle>$

BELG2 $\angle$

DOEIE: $L$

BOBटE : $\angle$

iSODE $L-$

उEDSE $\angle-$
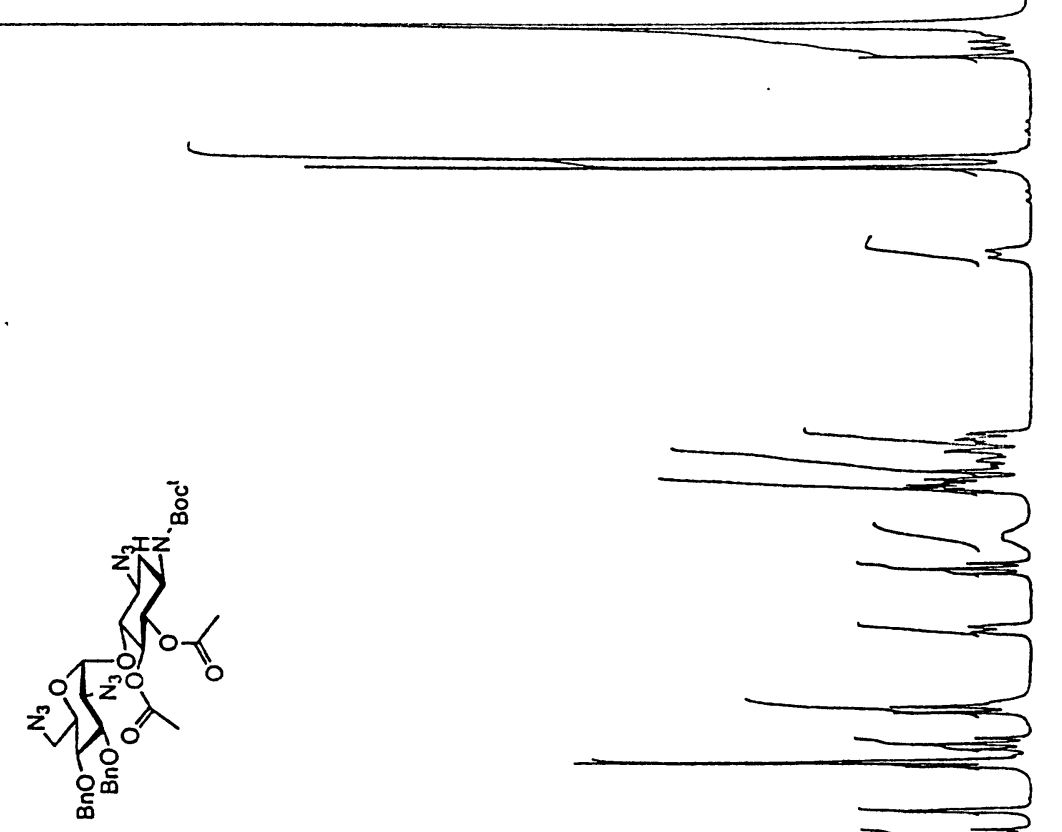

160.21

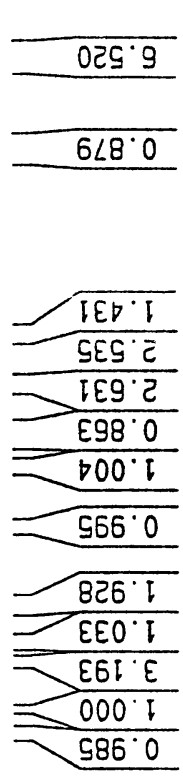




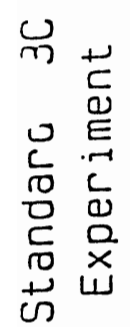

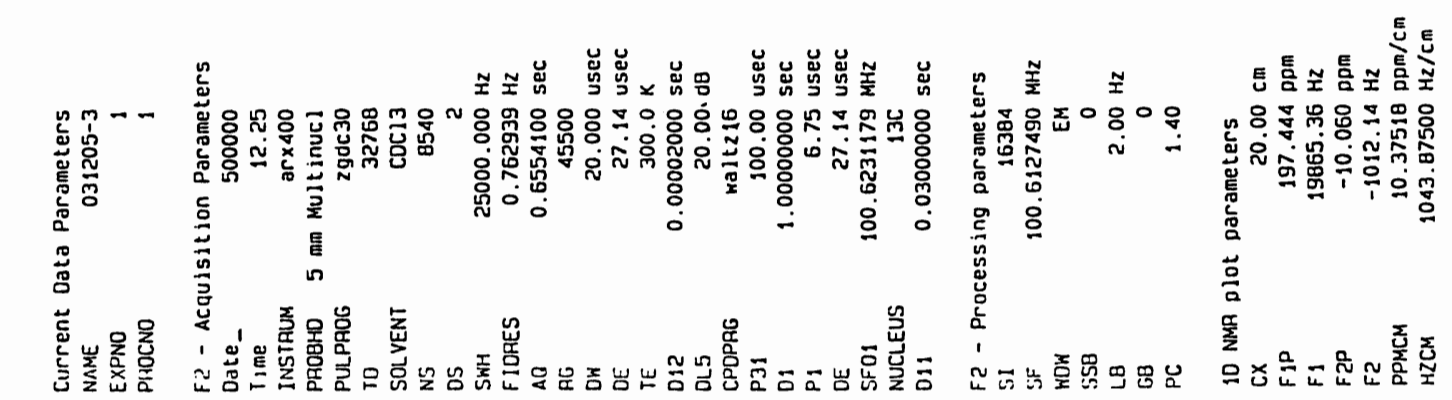

$\therefore$

$66 L^{\circ} 02$

$888^{\circ} 02$

$85 \nabla^{\circ} 82$

टB० $\varepsilon E$

ODI.6D

$\triangle 50^{\circ}$ IS

8ट2 65

8IS' $\varepsilon 9$

6ES' $I L$

$\checkmark 29^{\circ} \dot{E L}$

टO० $\mathrm{C} L$

SES' $S L$

$968^{\circ} 9 L$

टIट ' $\angle L$

OES $\angle L$

$916 \cdot 8 L$

$86 I^{\circ} 6 L$

$\varepsilon \sigma L^{\circ} \sigma L$

$85 \nabla^{\circ} 08$

จ $62 \cdot 66$

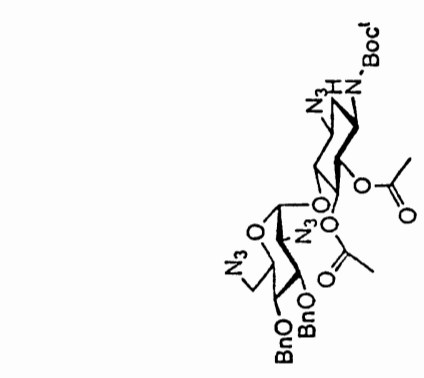

SL6 $\angle 2 I$

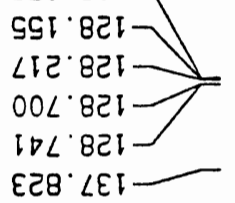

2SI'SSI -

$80 E^{\prime} 691$

टEE $I \angle I-$ 


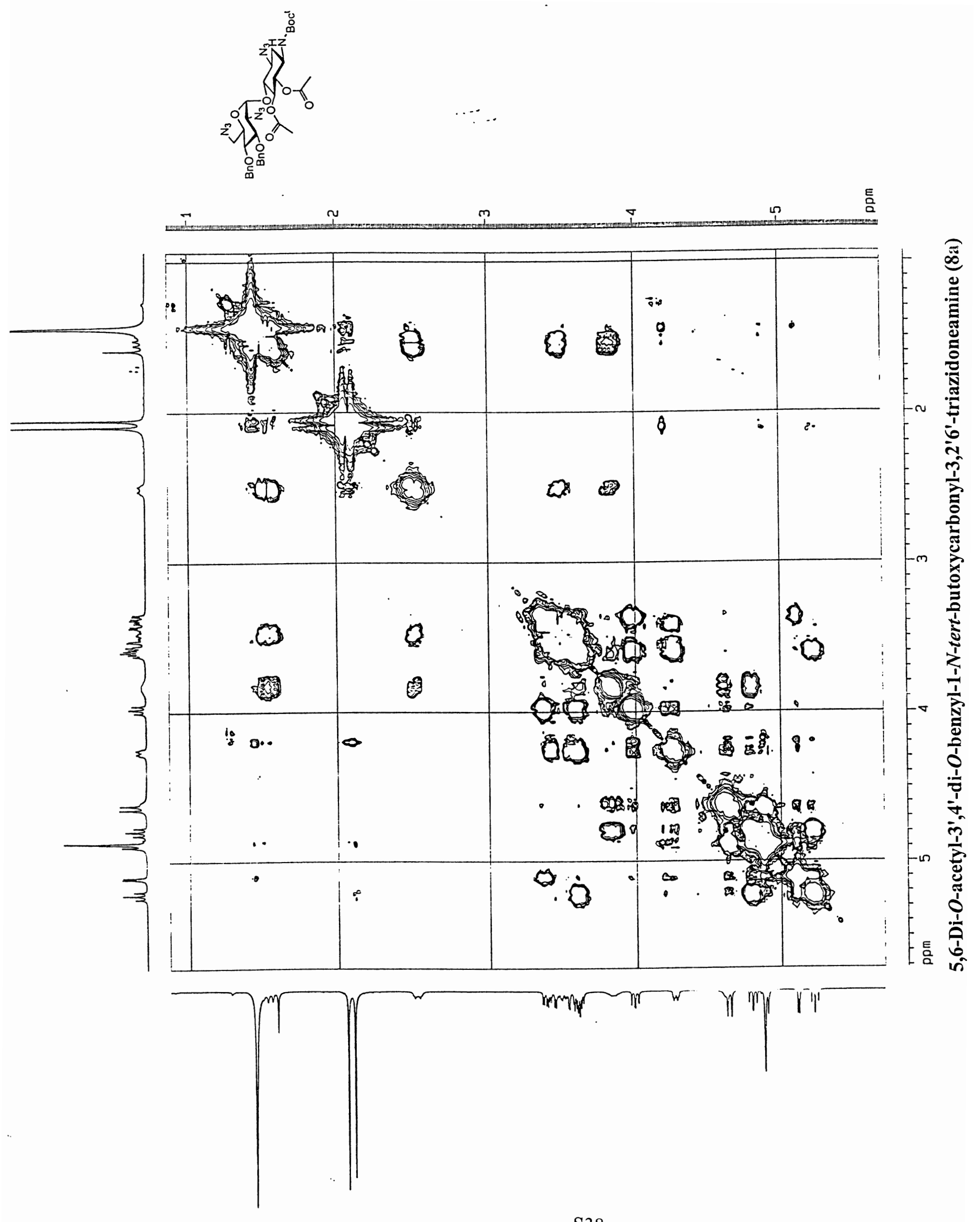




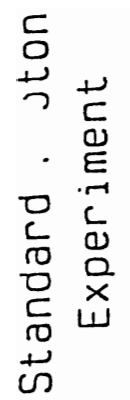

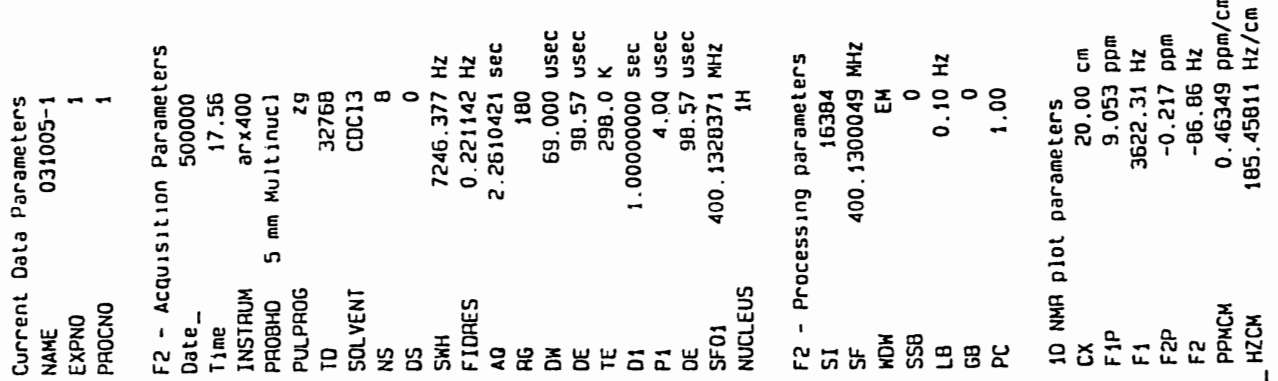

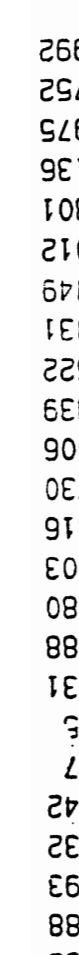

266[2. โ

ट૬८Еट' []

SLGLE' 1$]$

SEISE. [-

[O8[D. 17

5100L. $17 \|=$

60050.5

IEDEO 2

एट960. 57

6EBOD. 57

$90080^{\circ} \cdot 27$

OELटE' $\varepsilon-$

GIGEE' $E-$

EOESE'E]

OBट9E $\left.{ }^{\circ} E\right]$

$882 \nabla S^{\circ} \varepsilon-$

IE89S $\varepsilon]$

उट65 $\varepsilon>$

$\left.\angle 009^{\circ} E\right)$

$2 \nabla 029^{\circ} \mathrm{E}$

टEB88. $\varepsilon$ $\varepsilon$ -

B8EI6. $E-$

$B 8 D \angle 9^{\circ}$

$60809^{\circ} \square$

टट558 $\circ$

$205 \angle B^{\circ}$

$8 D 288^{\circ}$

IE $\angle 06^{\circ} D$

GDCE6

$\subseteq \angle O D O^{\circ} \subseteq$

टEOSO $S-$

D89ST $s-$

$9 \angle 081 \cdot g-$

$08005 \cdot 5$

टSSEC ' $L$

66BE2 $\angle 7$

$\angle$ LSES

ED8S2 $\angle>$

ISOLC $\angle$

DEZGE' $\angle$

$\subseteq \angle E O E^{\prime} \angle-$

OZEIE.

O80टE $\angle$

उढटE' $L$

उ8ВEE

I टESE $L$

$S D S \angle D \cdot L-$

wdd
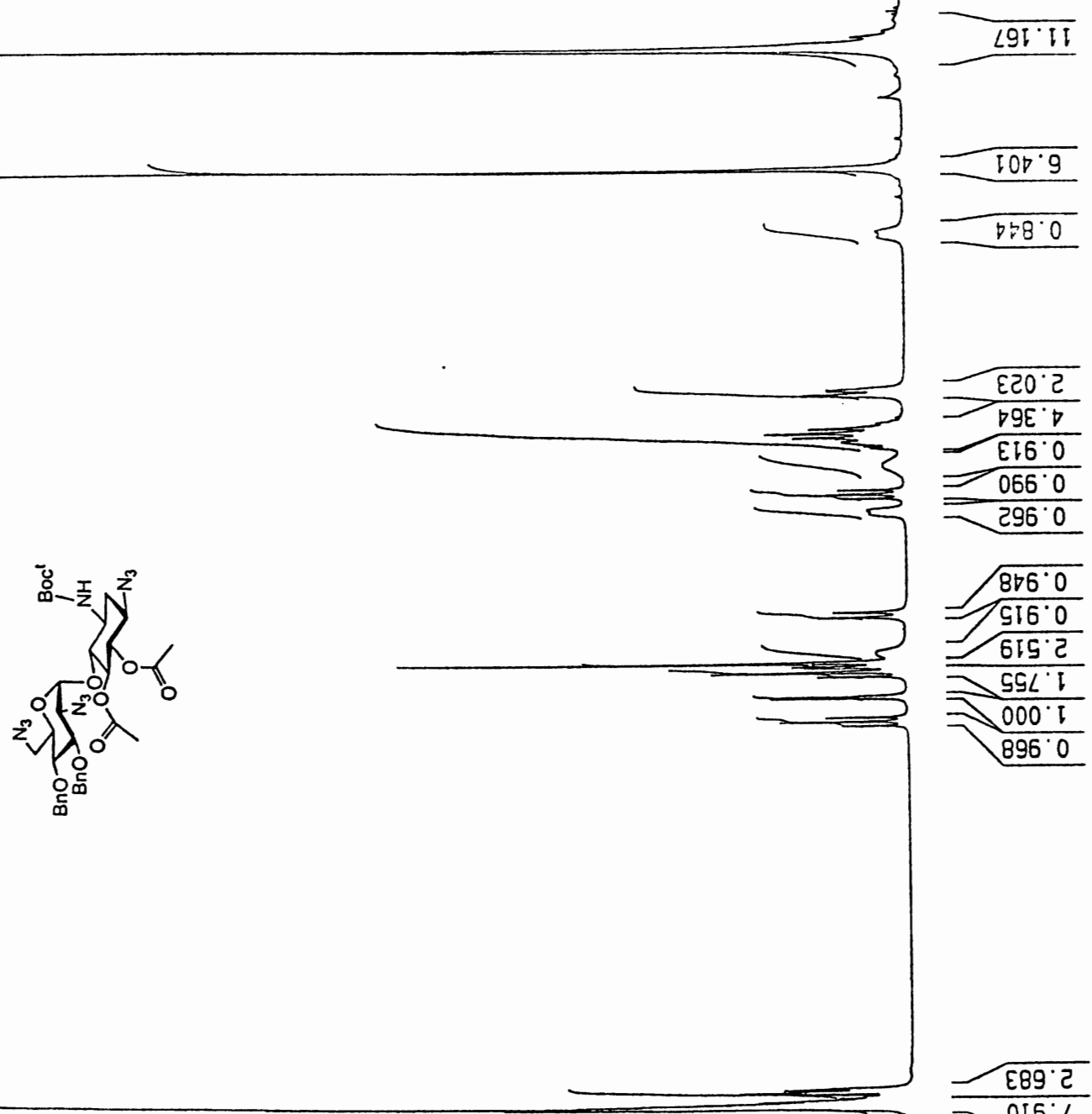

$016^{\circ} \mathrm{L}$

[ej6ajuI 


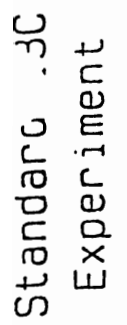
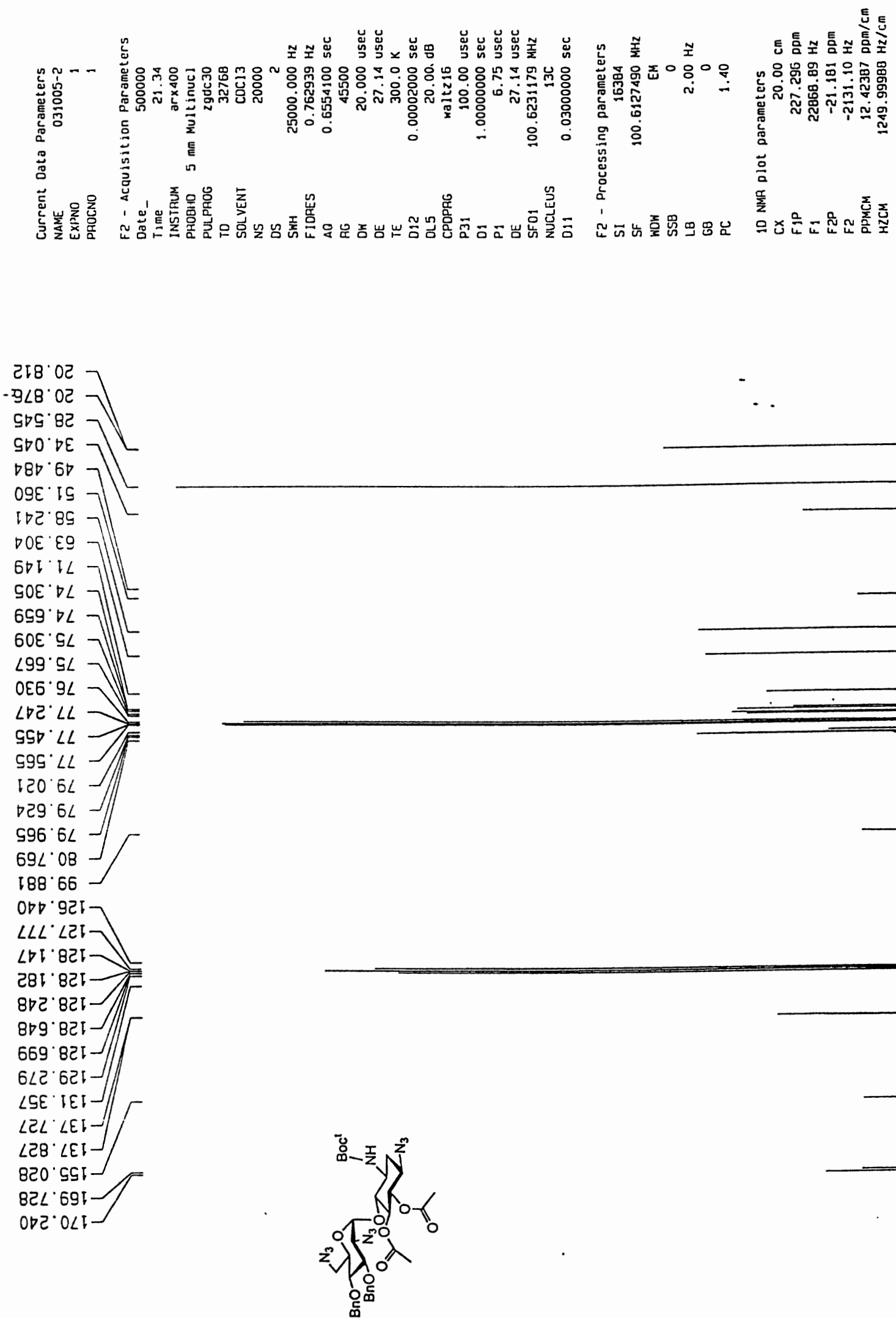

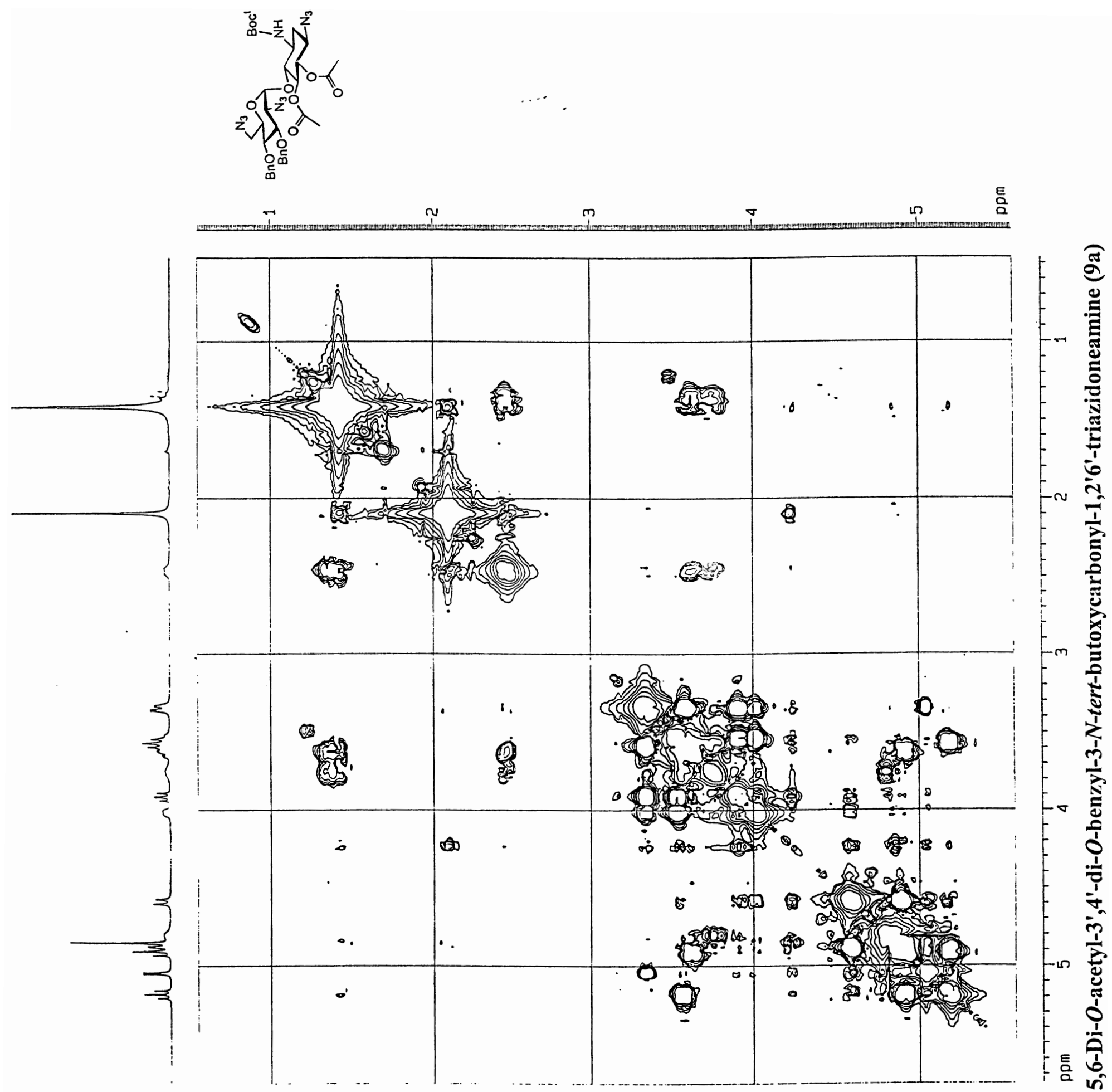


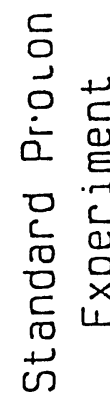
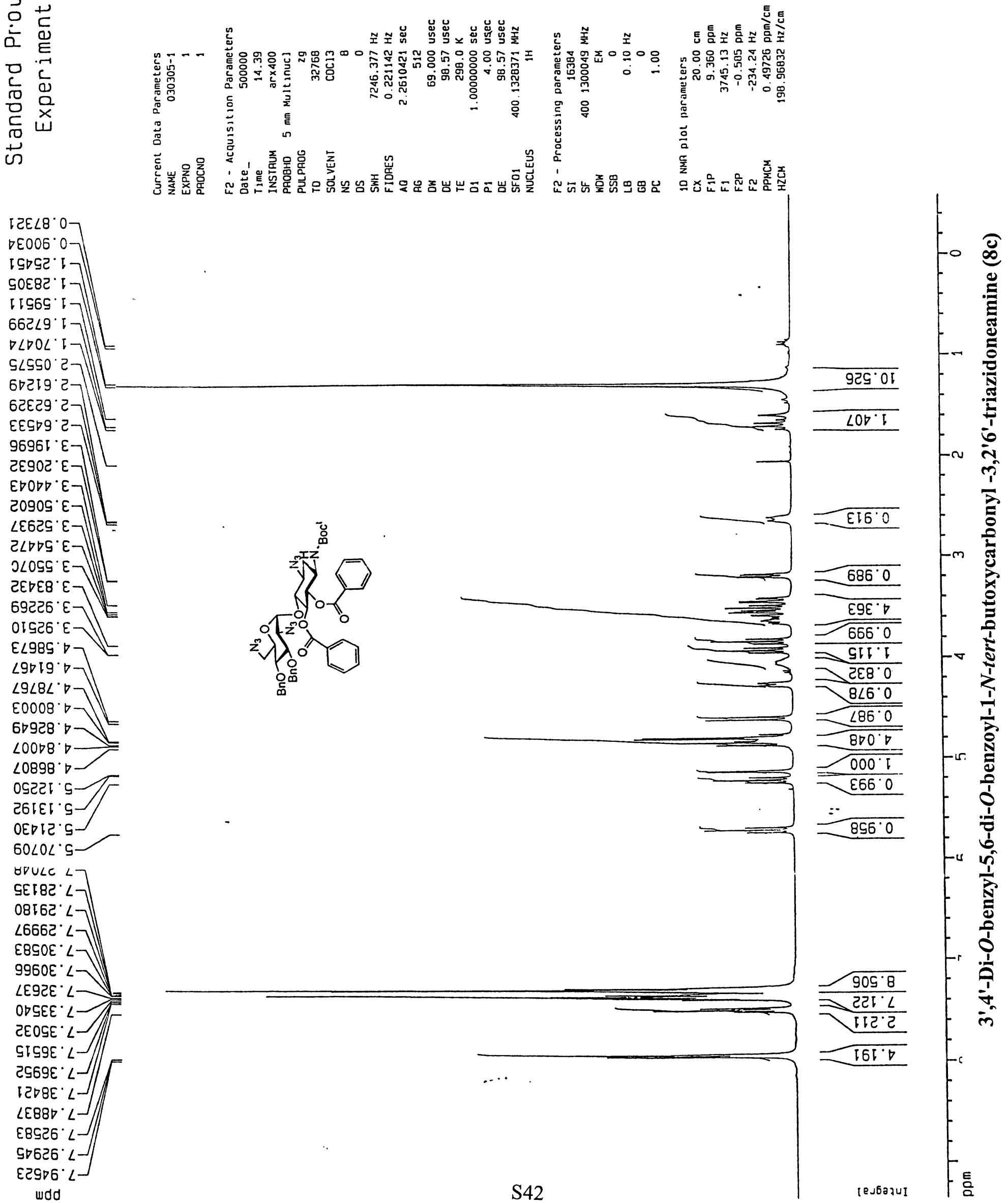

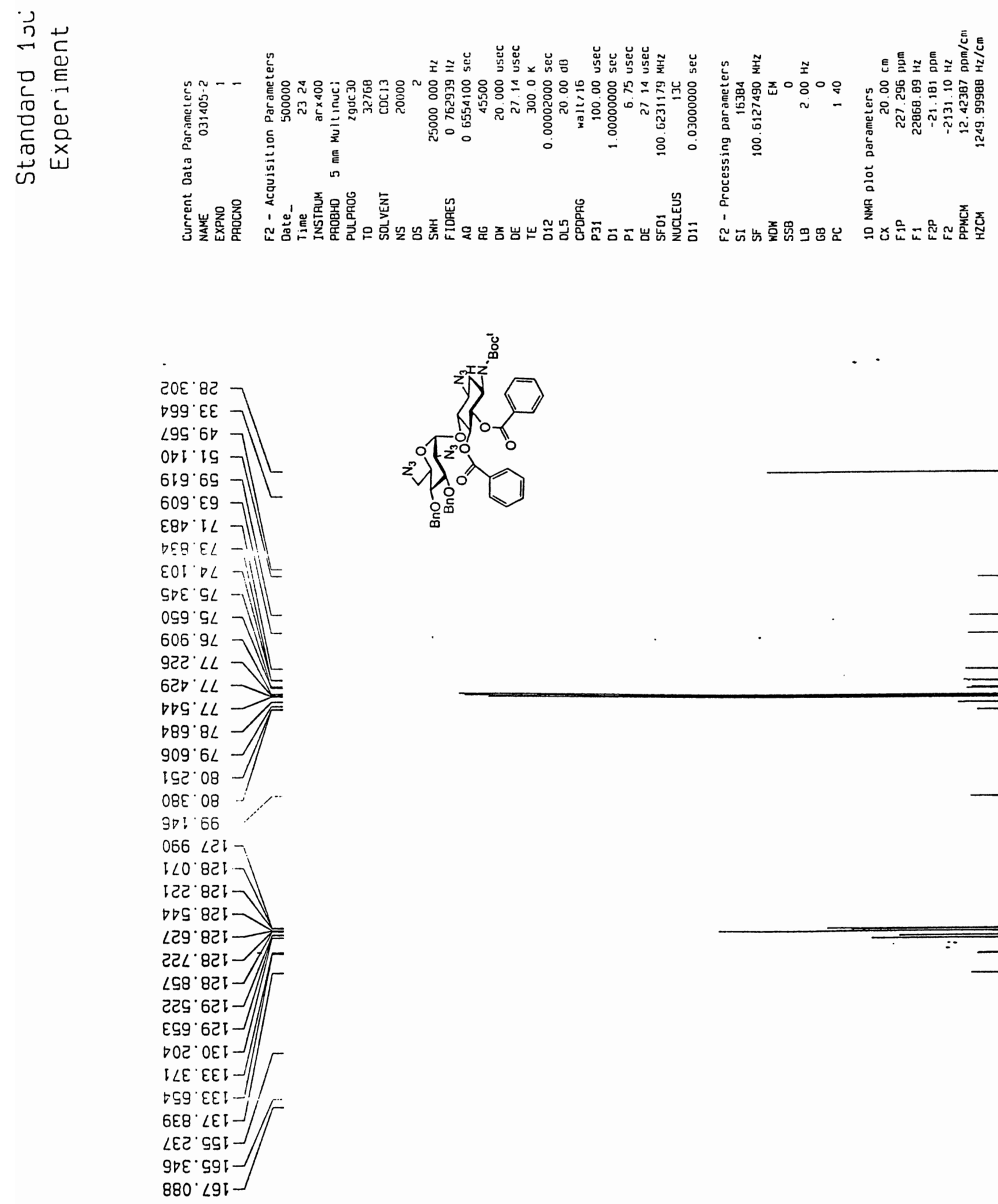


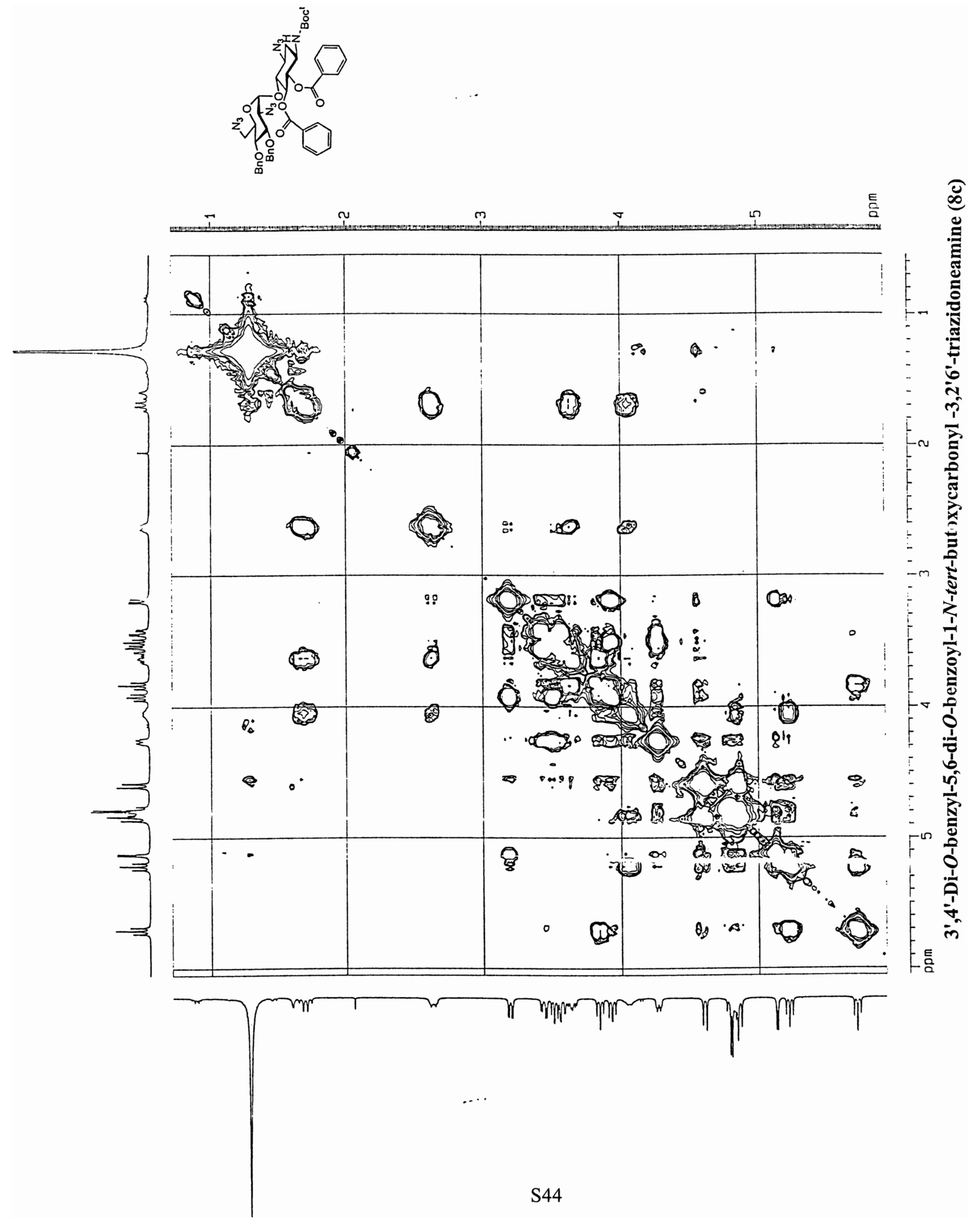




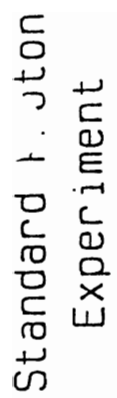

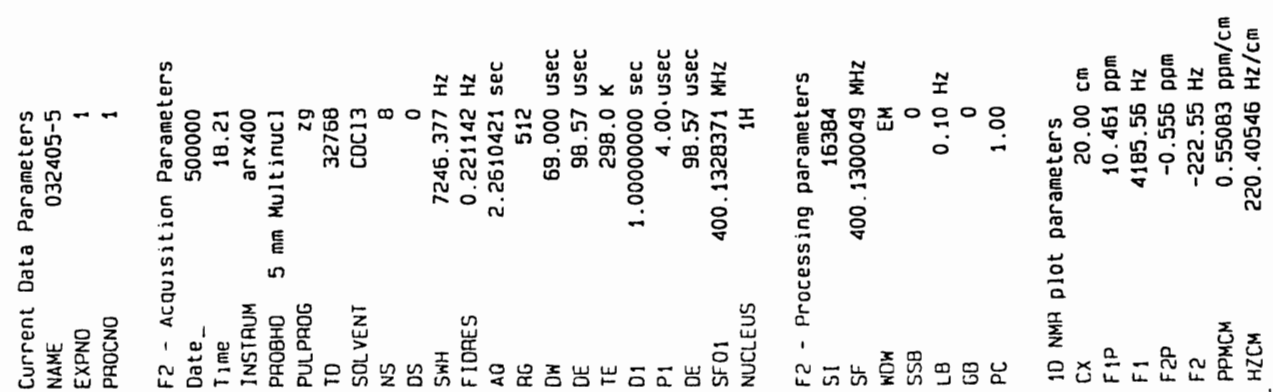

\section{$\angle I 8 \angle 8^{\circ}$}

EDटB8.0

S9८88.0

$\angle 9 \angle 68^{\circ} 0-$

$8 I \angle L C^{\prime}[7$

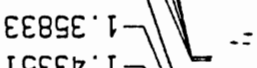

ISEED

$\angle \angle B D D$

6LCOL

EgDEL

IELEC $E$

$\angle B 9 D C^{\prime} E-$

SEE9ट ' $E$ -

$\angle S C \angle C^{\circ} \varepsilon-$

$\left.869 \nabla S^{\circ} E\right]$

$\varepsilon$ เรट० $\varepsilon]$

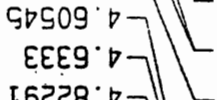

โ

¿ $\angle 6 \circ 8^{\circ} \circ 7$

$99058^{\circ} \circ 7$

$01288^{\circ} \cdot \sqrt{ }$

$65988^{\circ} \cdot \mathrm{V}=$

SSOI.

0द662.5 -

จE689.9-

$060 \angle C^{\prime} \angle-$

$0 \angle 68 C^{\prime} \angle-$

EEDGट ' $\angle 7$

$9 \nabla 20 \varepsilon^{\circ} \angle>$

I LCIE: $\angle 7$

EDOZE' $\angle-$

O9LCE ' $\angle$

IOEEE $\angle$

$\varepsilon \angle B E E \cdot \angle-\mid M$

टLSLE $\angle-/ \|$

$998 \angle \varepsilon^{\circ} \angle J$

$8 D 810 \cdot \angle-$

SLOCD $\angle-$

$\varepsilon \sigma \angle L b \cdot L-$

$2086 L^{\circ} L-$

$\angle D 818^{\circ} \angle$

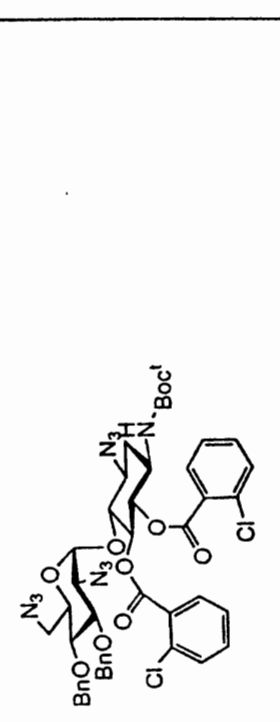

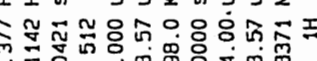

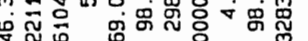

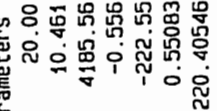

음

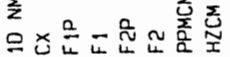

$06069^{\circ} 6$

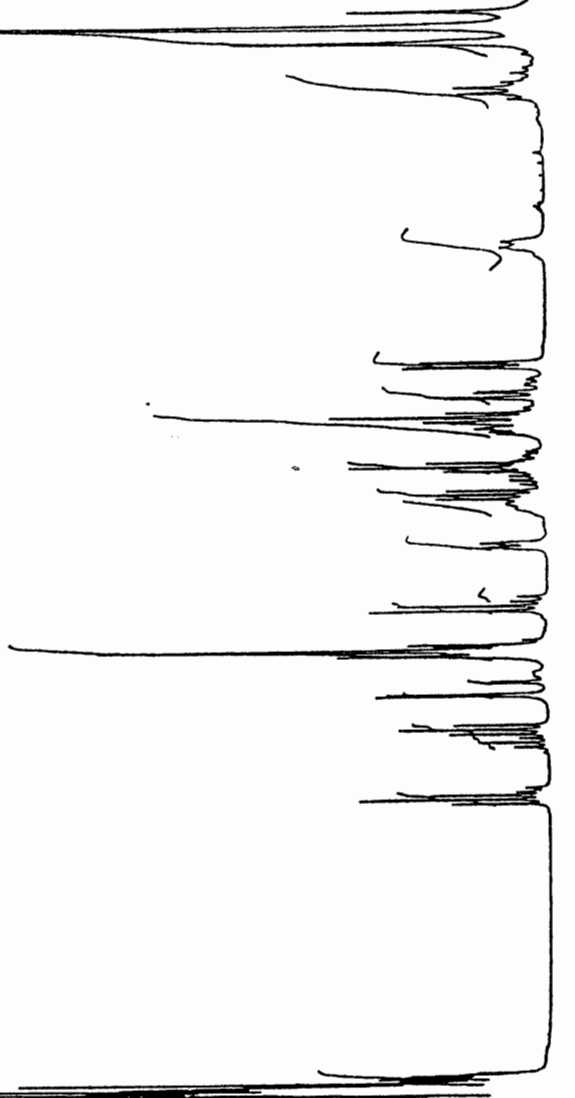

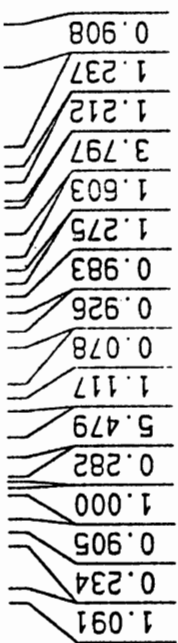

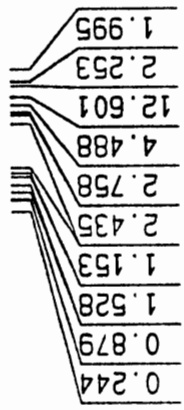

wdd 

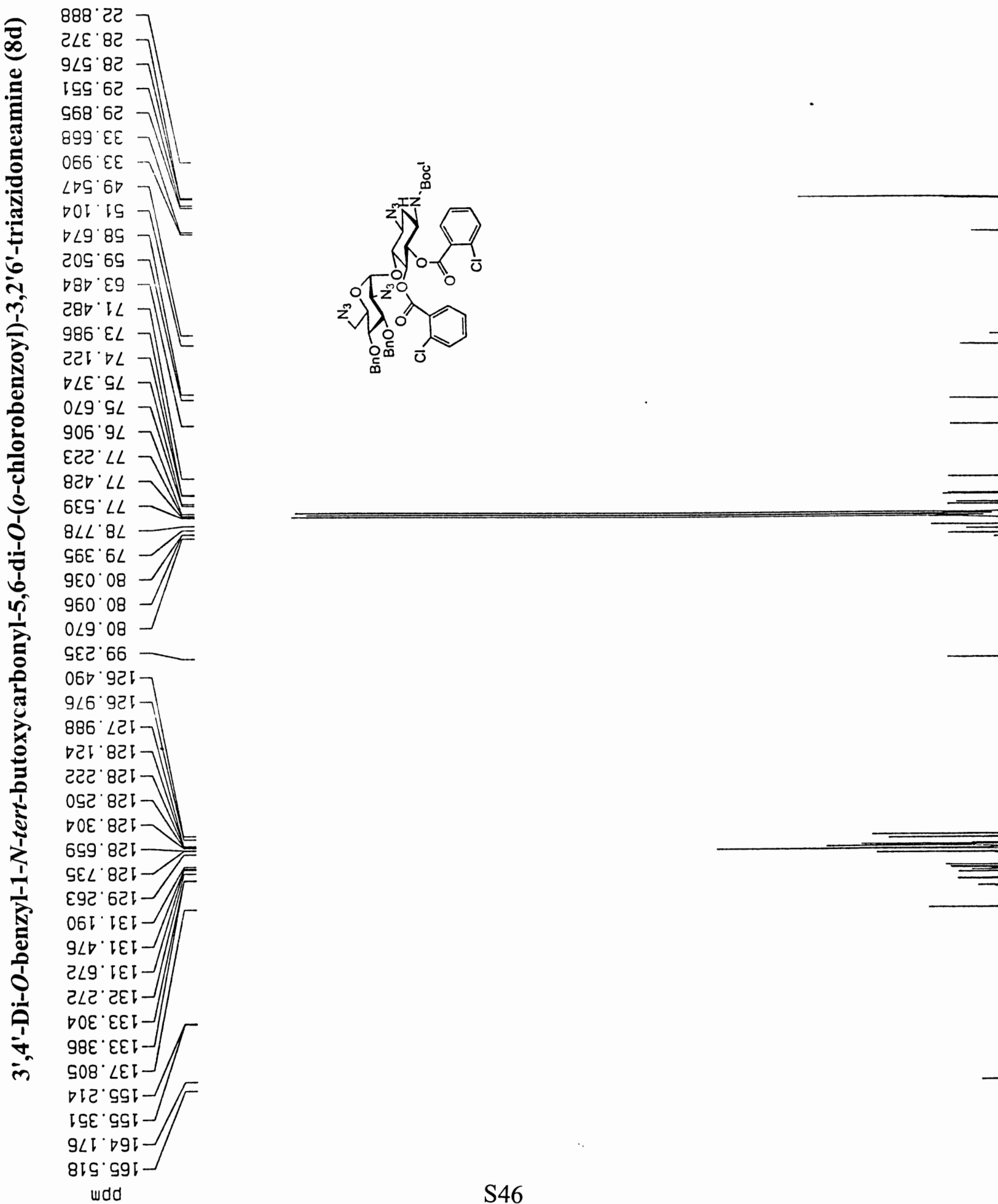


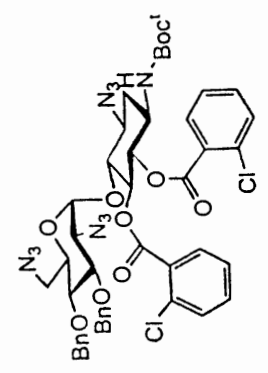

N

$m$

$\nabla$

(n)

틍

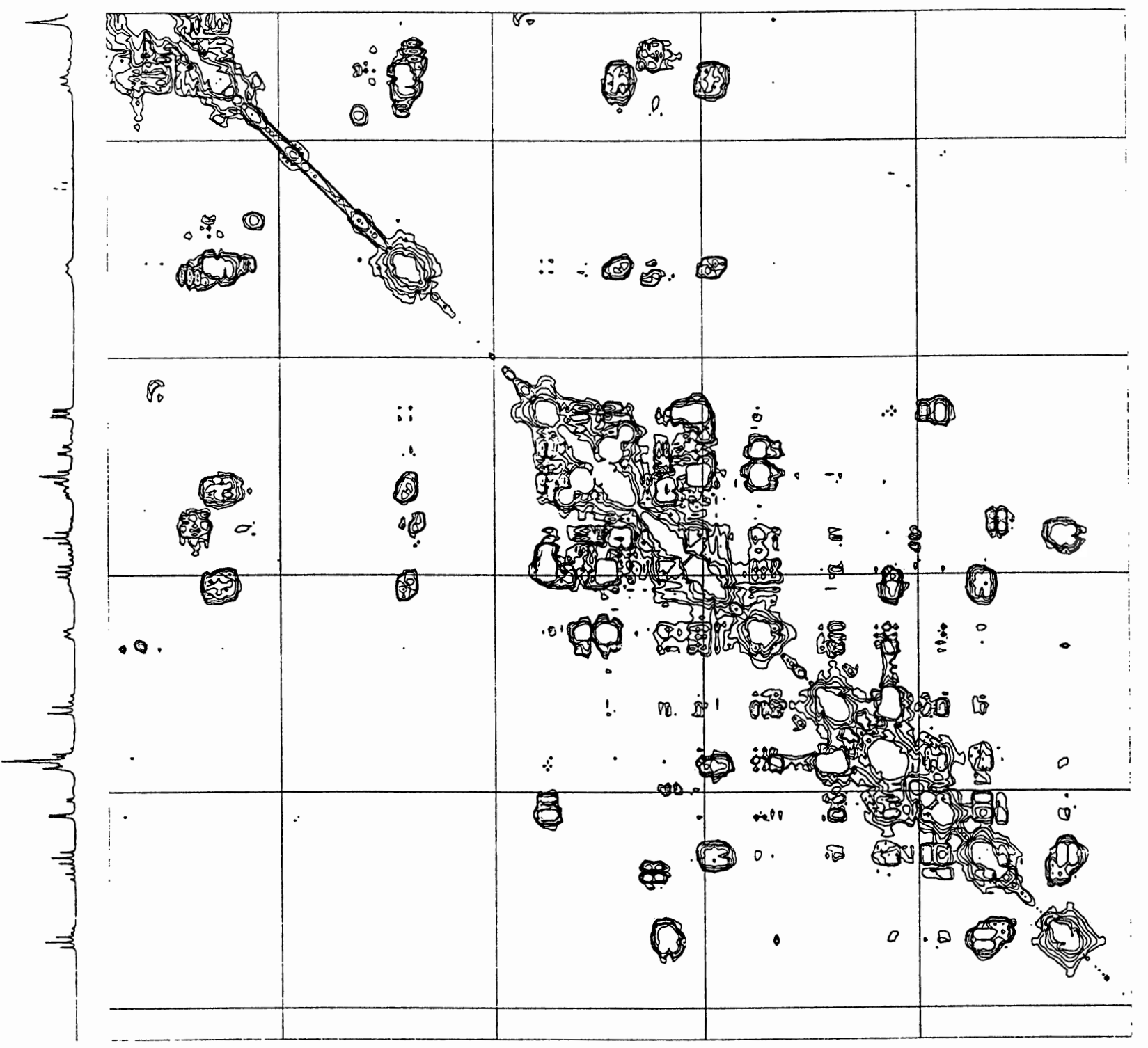

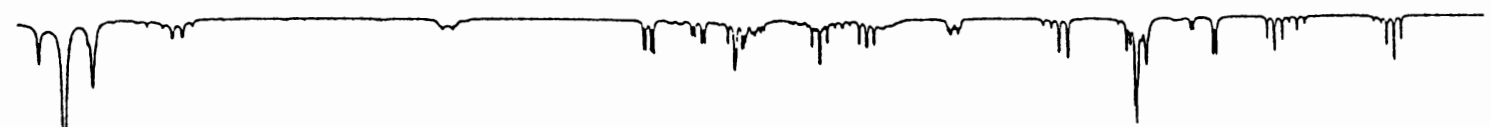


돈

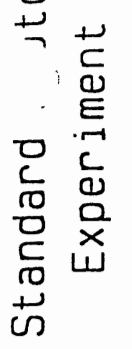
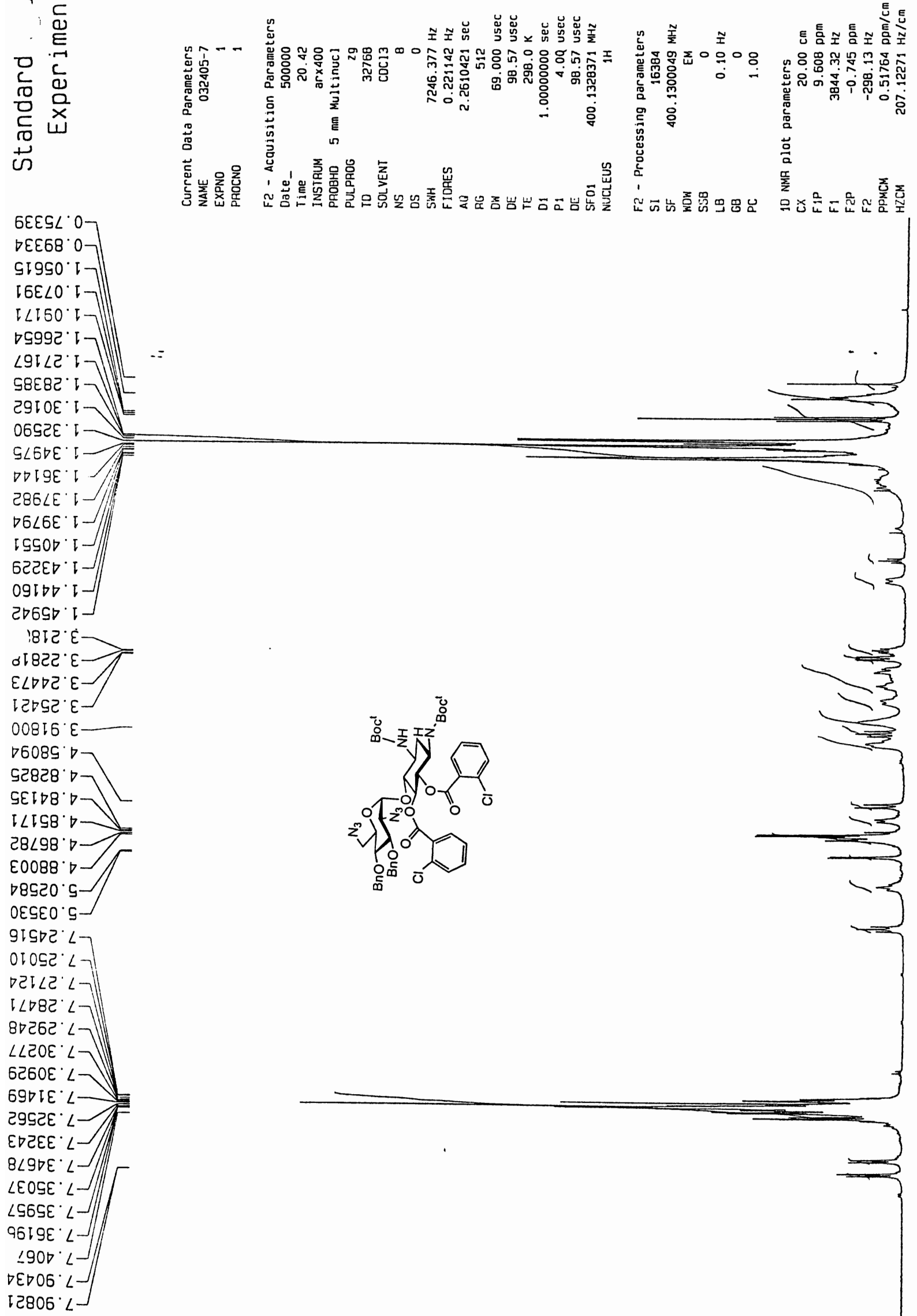


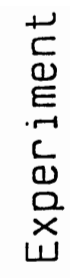
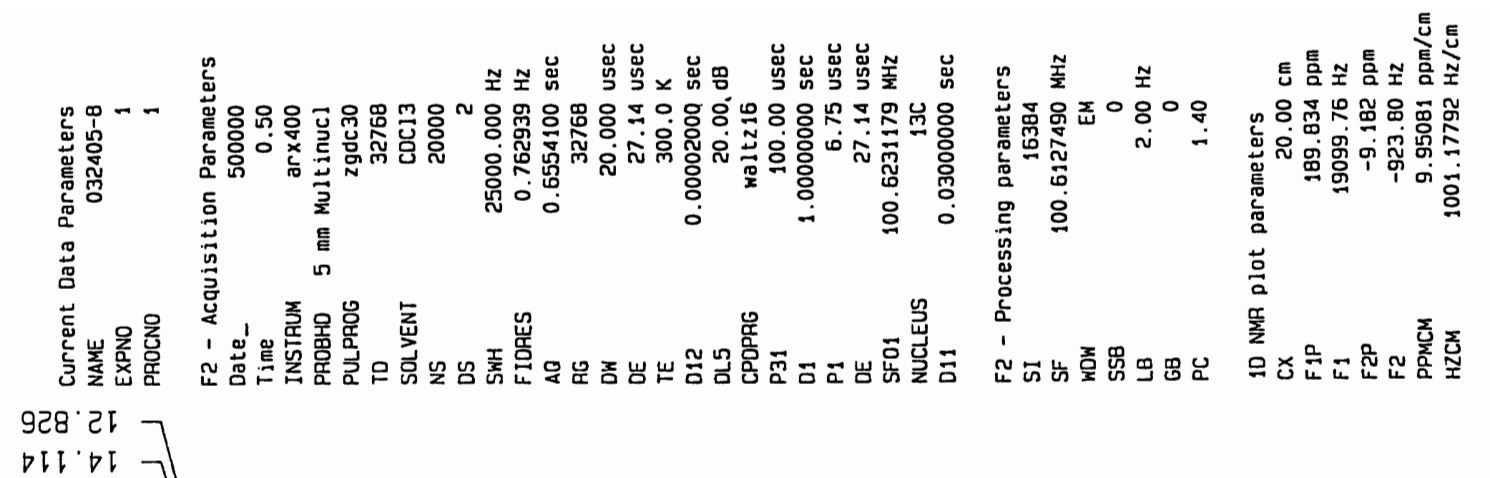

$\triangle 6 ट \cdot \square[7]$

$\angle \angle 8.22$

65E. 82

ट $269 \cdot 82$

$879^{\circ} 62$

$588^{\circ} 62$

9L6 IE

II. $2 E$

ह8Г. $6 \varepsilon$

$068^{\circ} \mathrm{D}$

टट9 60

BDE. IS

06० $\varepsilon 9$

SOI' $L$

8EG' $\nabla L$

हटा $\nabla L$

$G \nabla \mathcal{C}^{\prime} \subseteq L$

$\varepsilon 0 L ' S L$

$568 \cdot 9 L$

टाट. $\angle L$

SID $L L$

OES 'LL

ट๑8. $8 \mathrm{~L}$

210.08

$298^{\circ} 66$

868.951

$98 I^{\circ} \angle \mathrm{LI}$

टOL $\angle$ LT

$\angle 9 L^{\circ} \angle 2 T$

980.8517

2
$\varepsilon I E .851-851$

८ट8.6टा

$\angle O O^{\circ} O E I$

¿ $\angle 0$ ' $E$ โ

$8 \nabla 9^{\circ} \tau \varepsilon \downarrow$

GDI'टEI-

8LE' टEI-

QII EEI -

ट8I.EE - $]$

$98 \angle{ }^{\circ} \angle E I$

$868^{\circ} \angle E I-$

E90 ५५ I

Е80 จ9เ

982 ร9:-
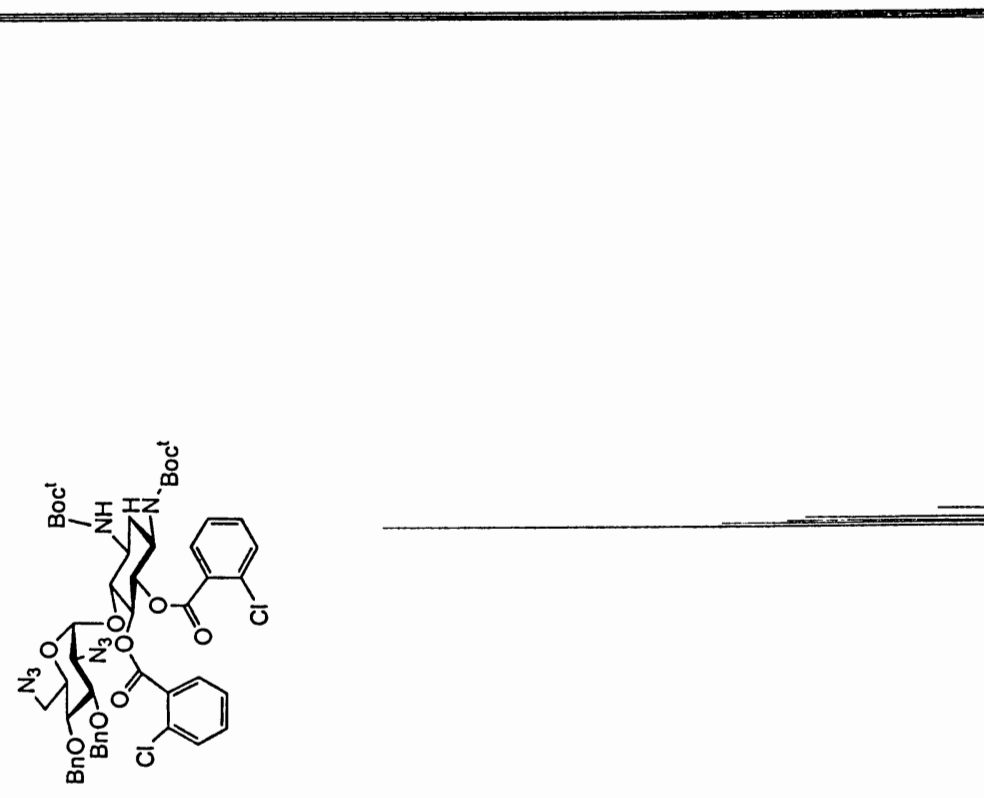
N

$m$

$\nabla$

틈
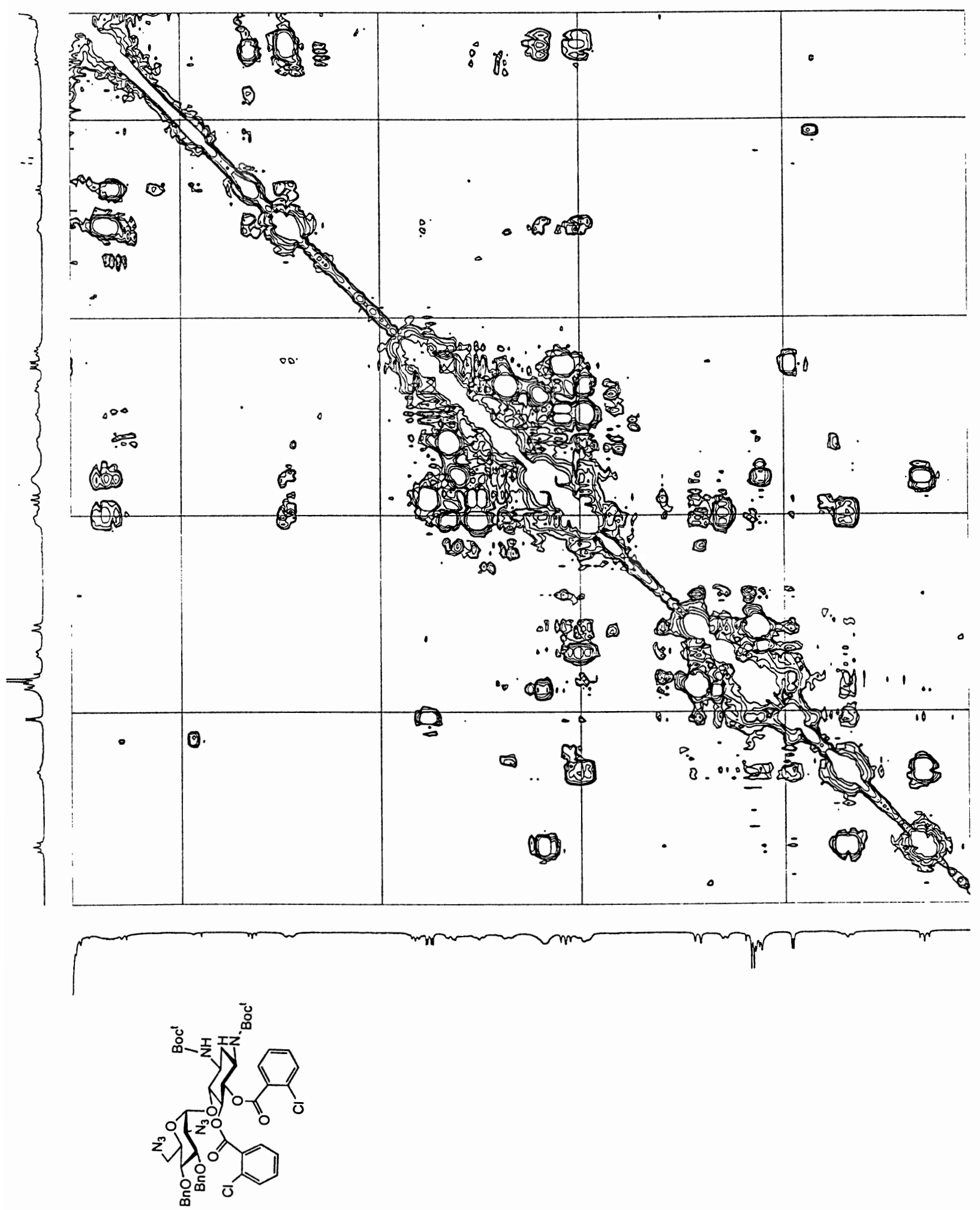

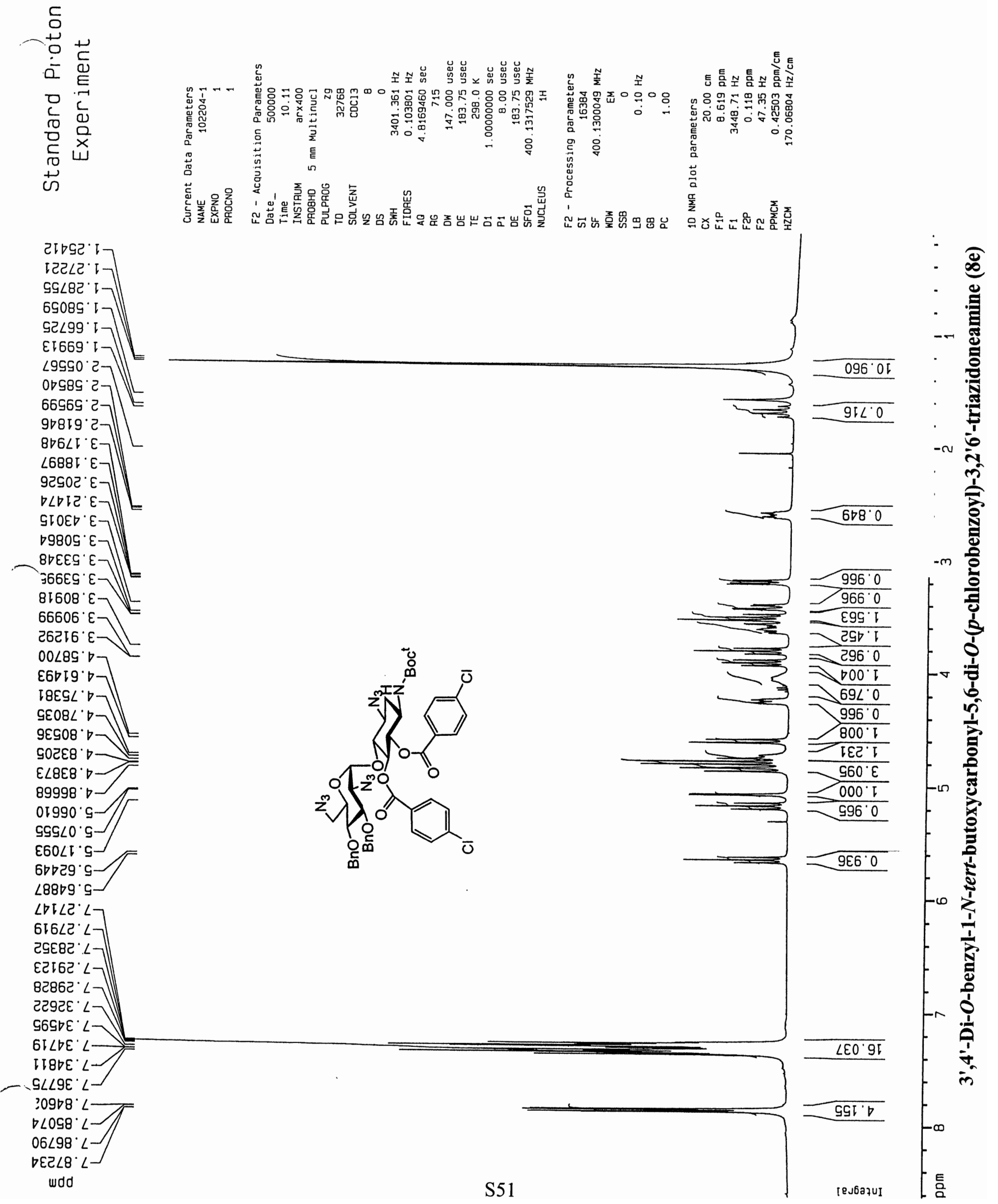


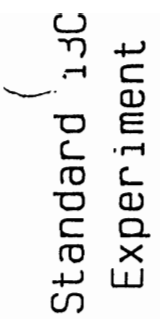
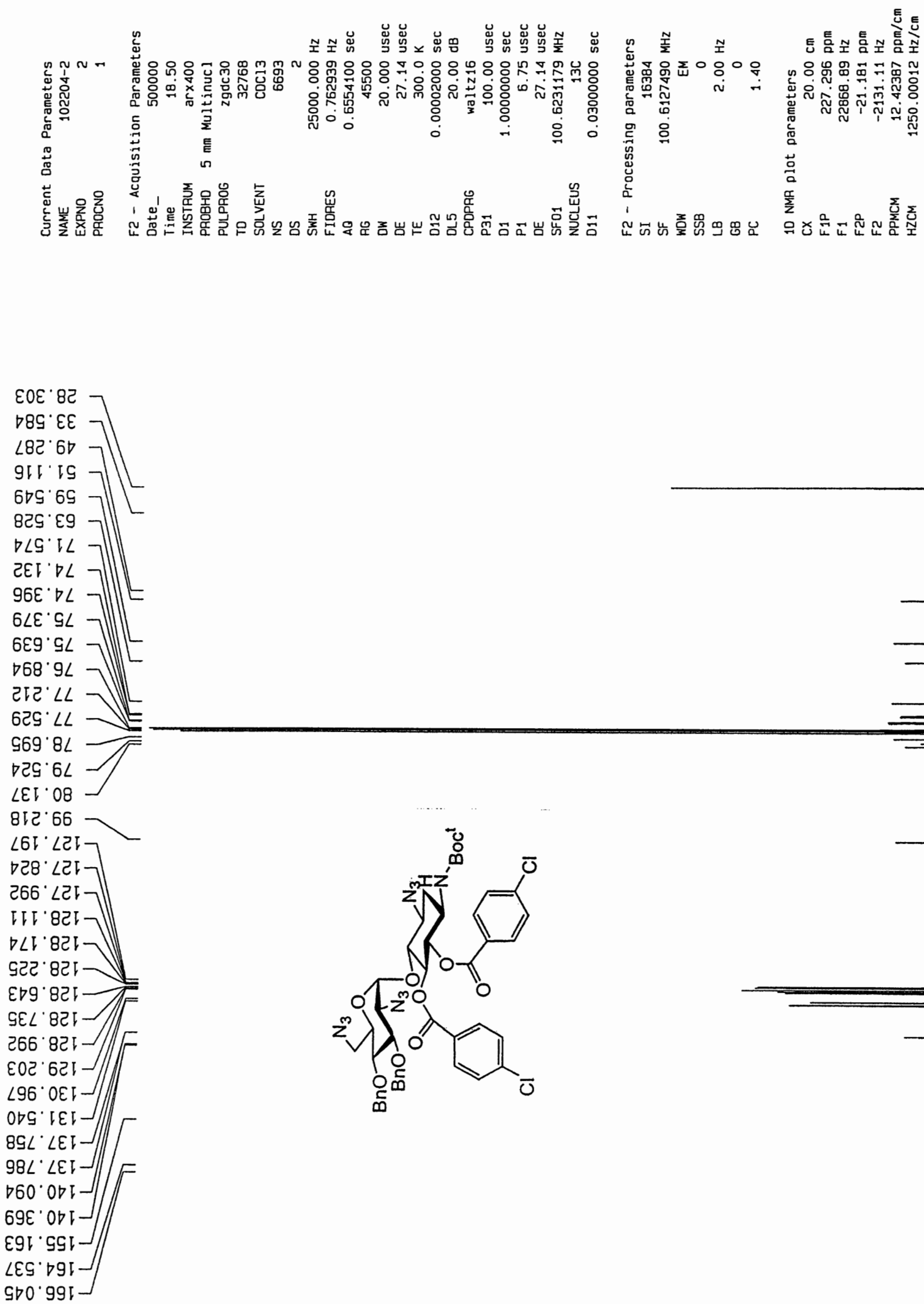


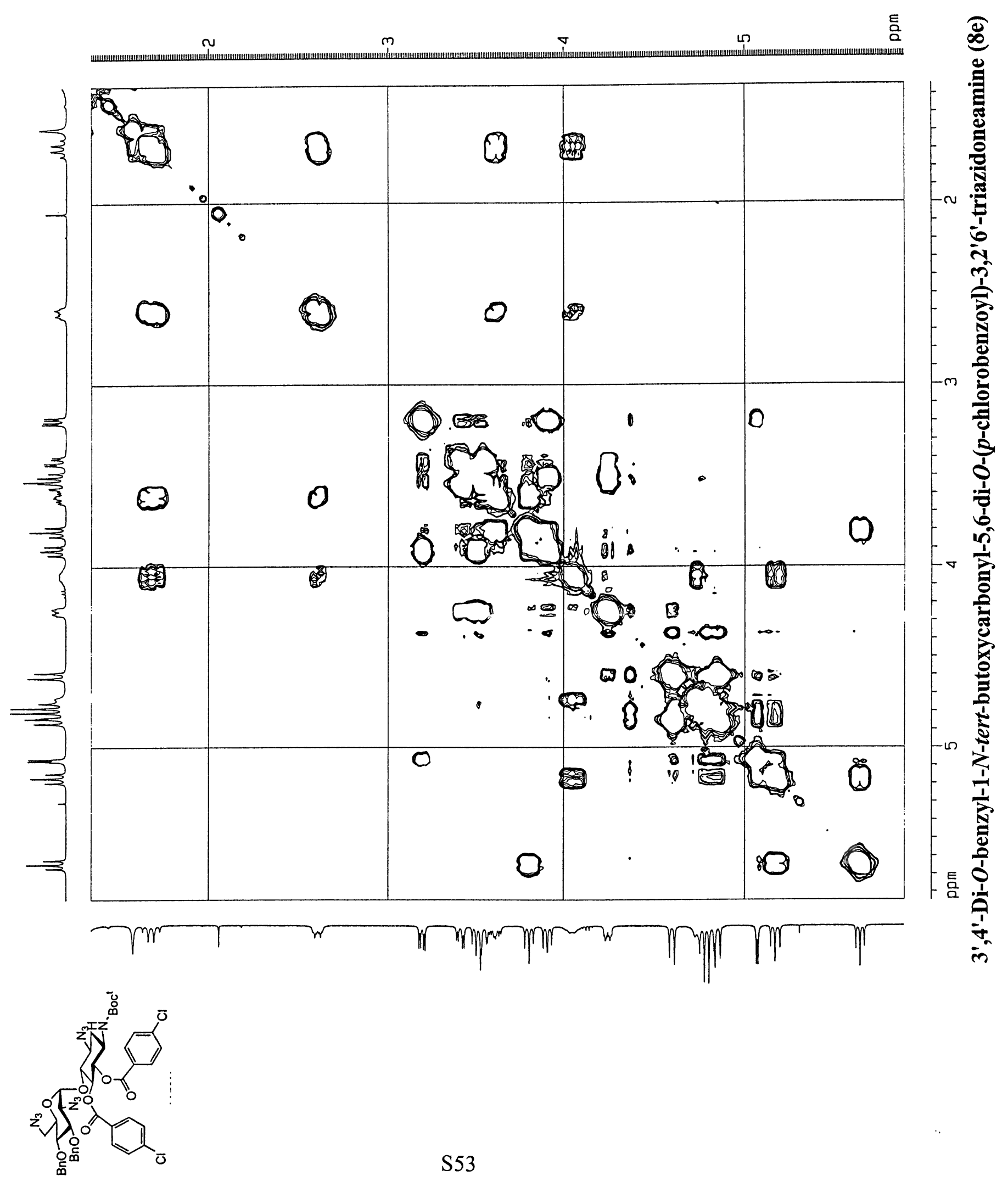




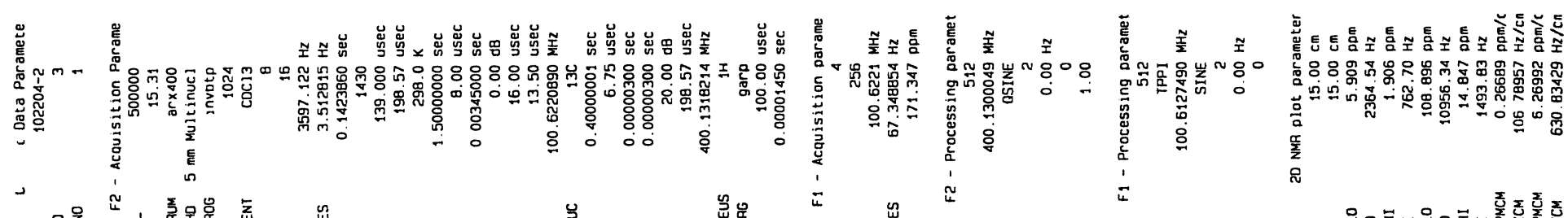

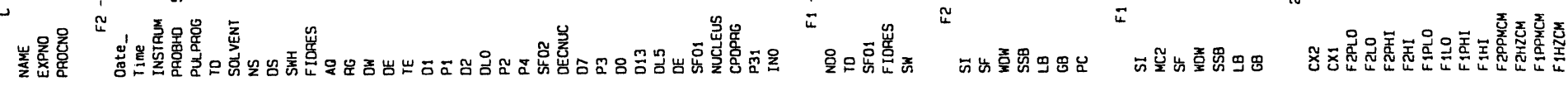
윰

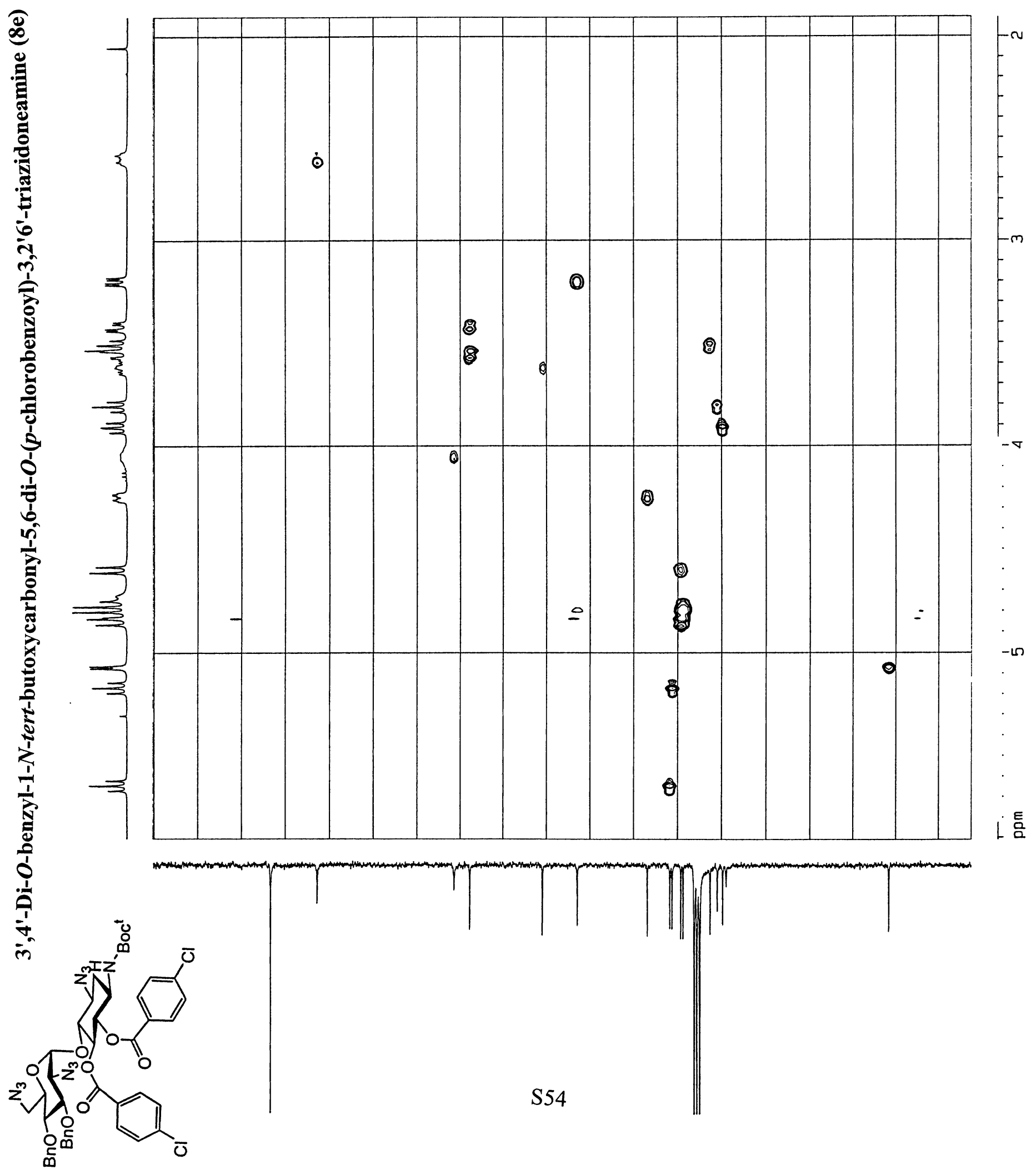




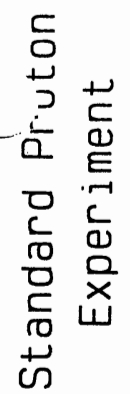
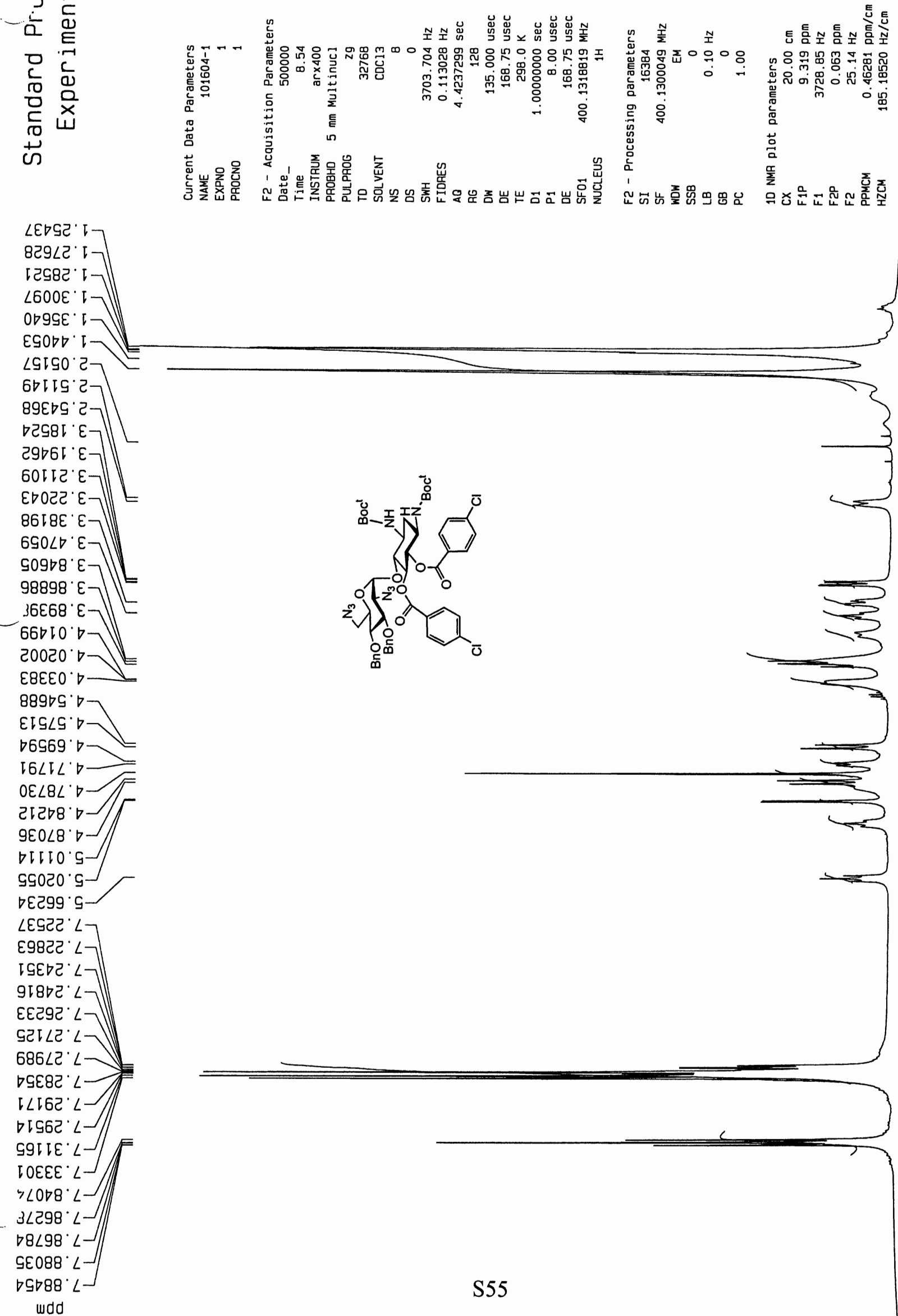


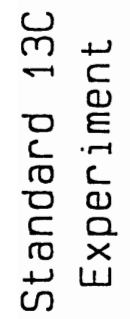
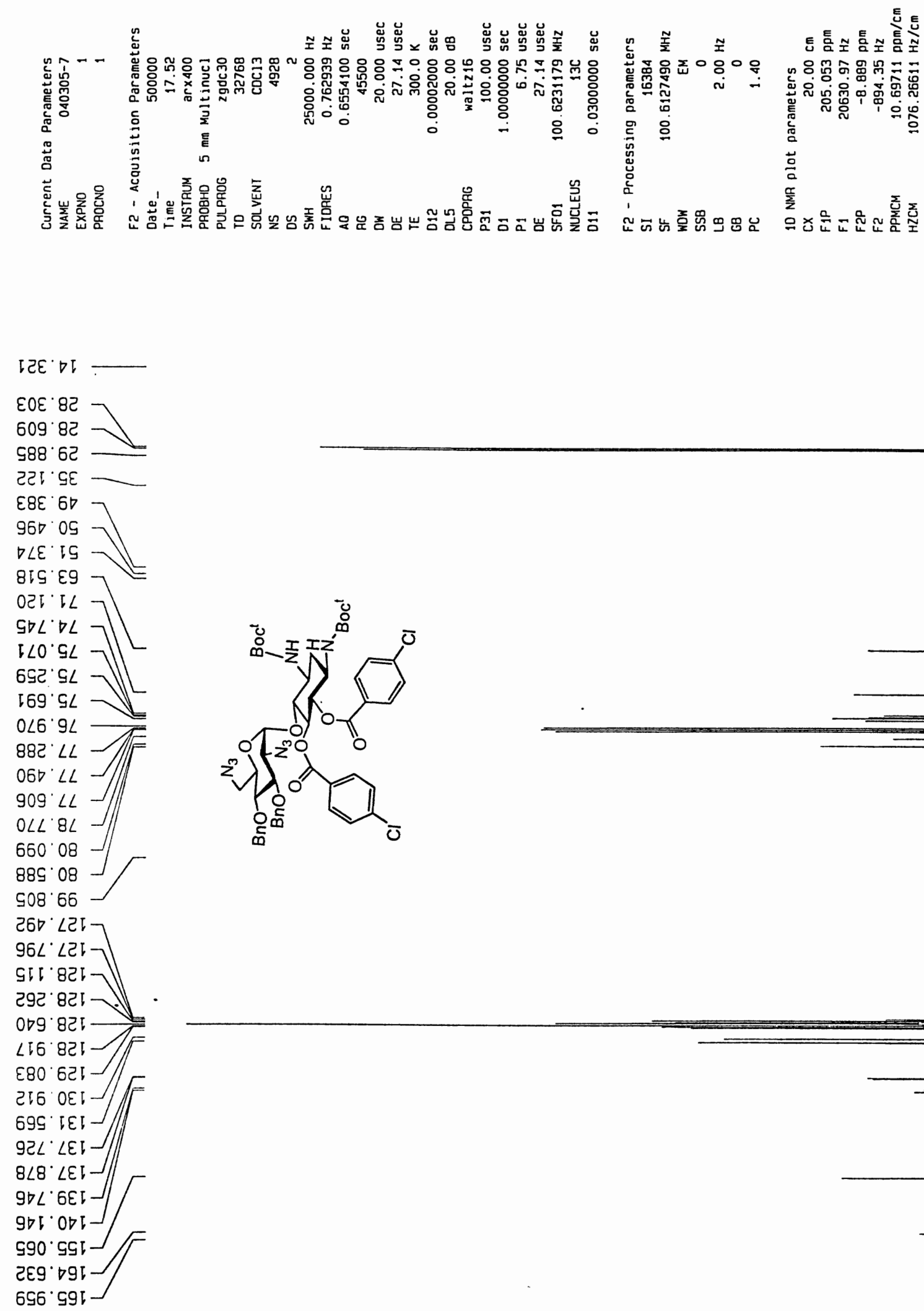


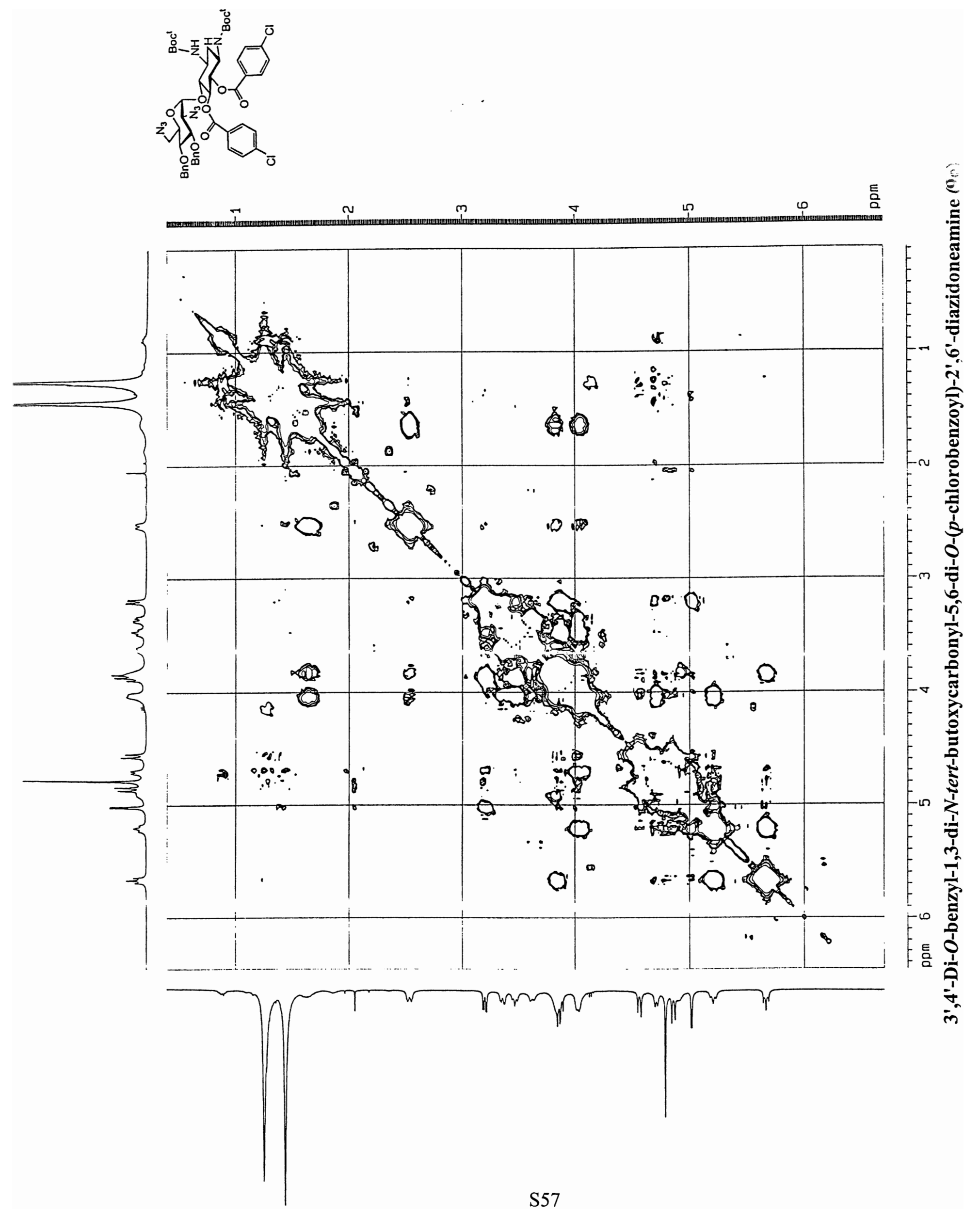



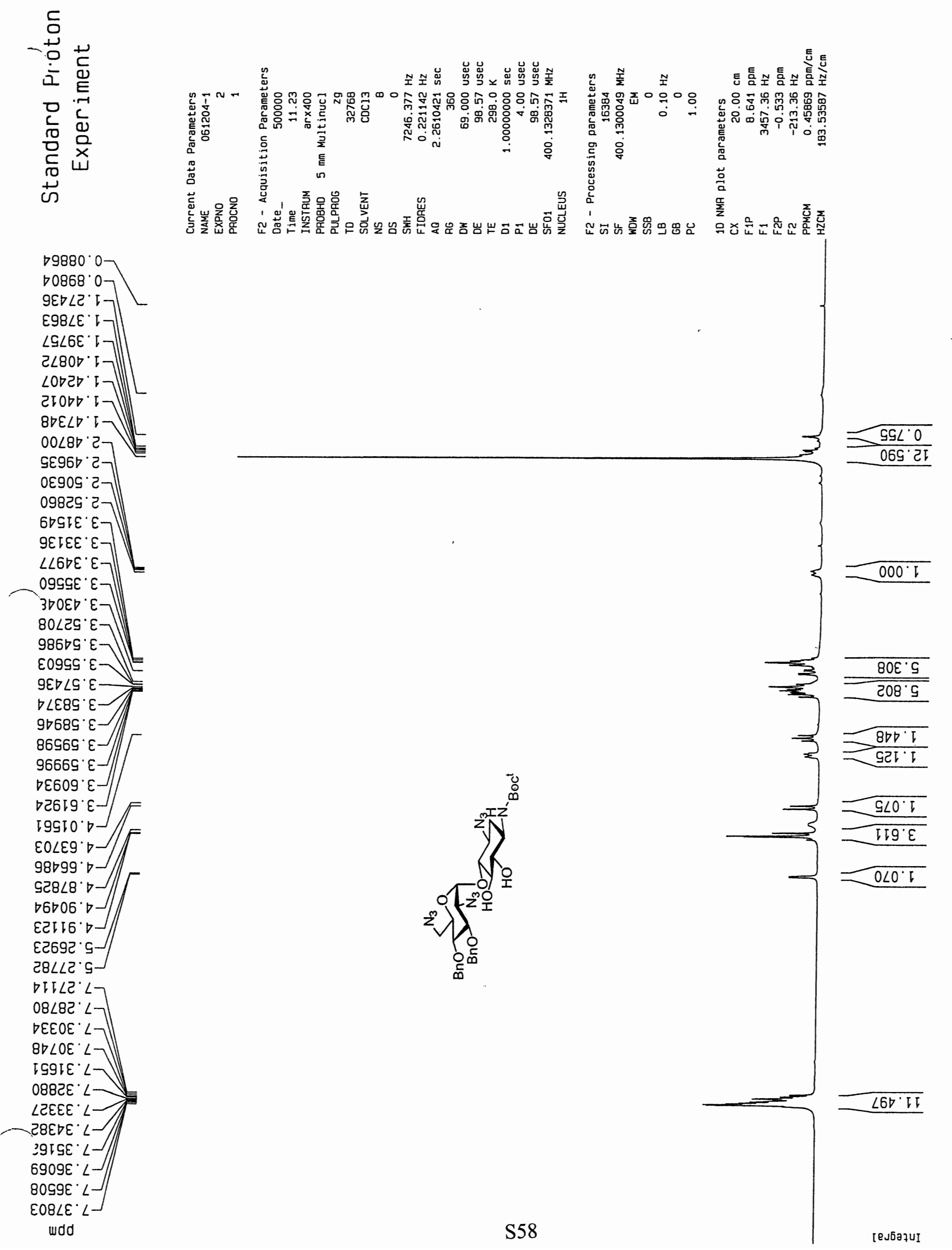


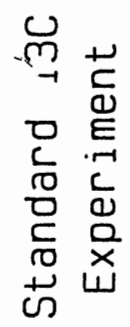
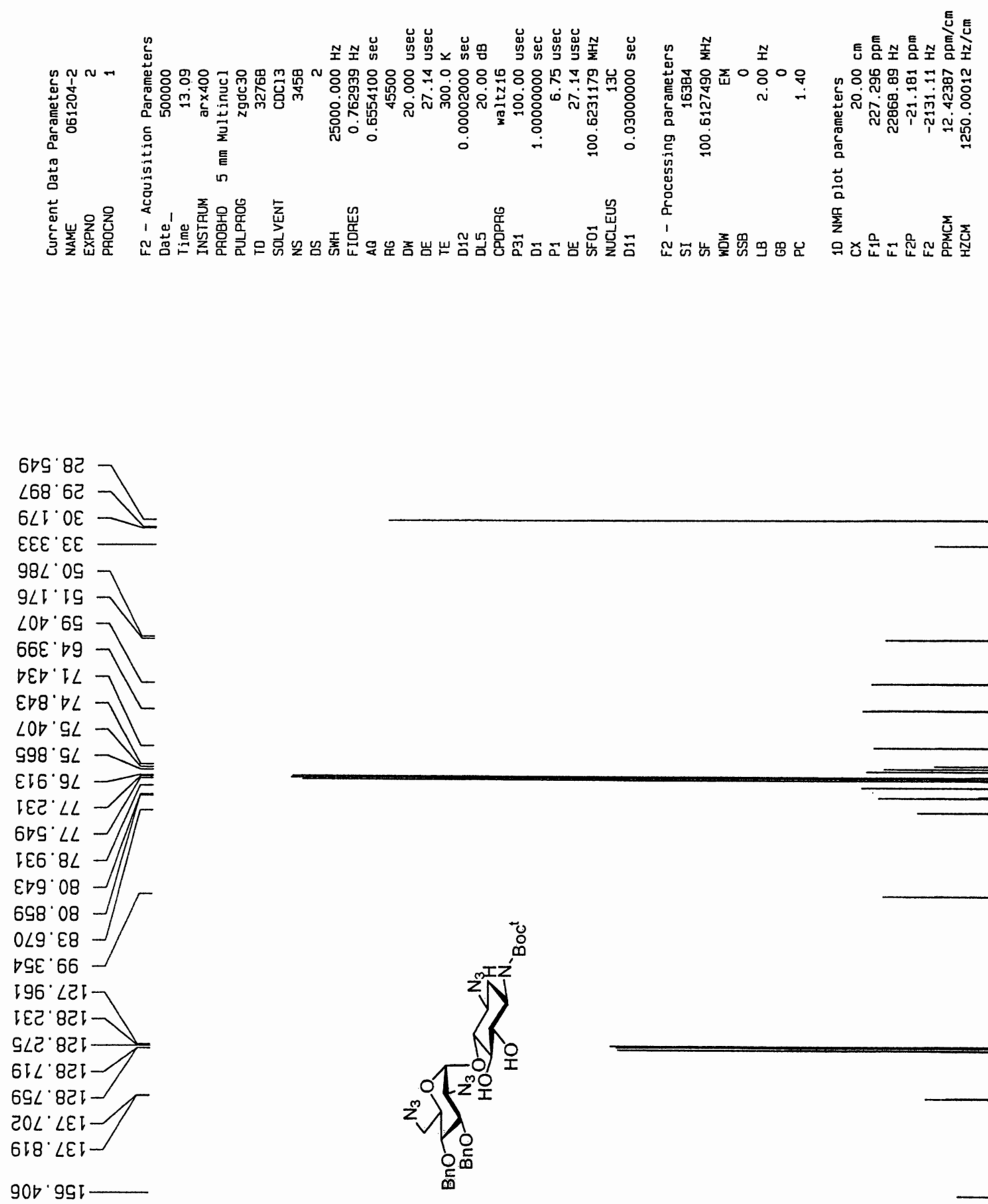


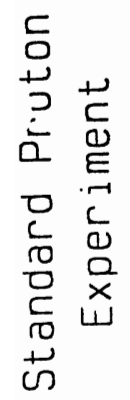

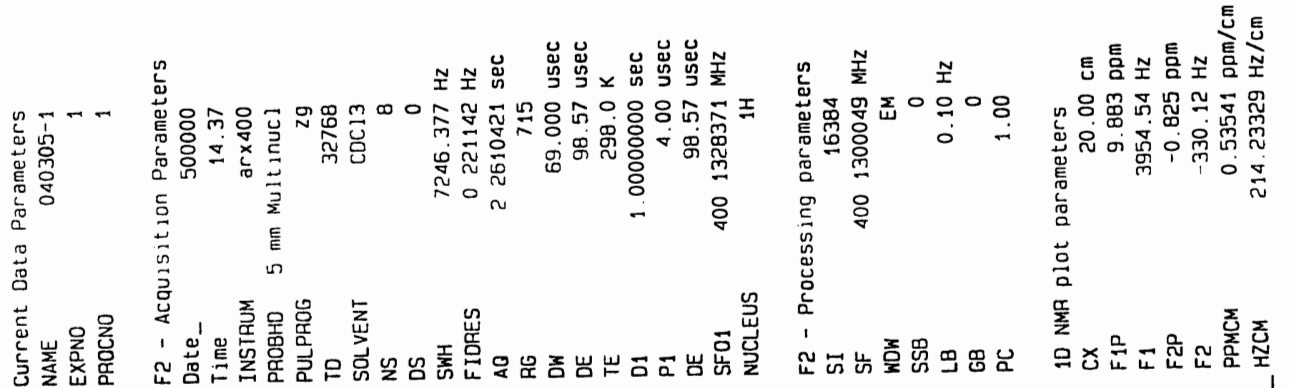

$\varepsilon \angle \nabla 80^{\circ} 0$

Sटा LC. []

88E8ट

88862 '

EOLटE

DIS6E

66000

ODI09

с898\% 2$]$ ᄂ

$09 ट ा \tau^{\prime} \varepsilon-$

8 8ร5० $\varepsilon-$

ЕโВED $\varepsilon]$

$\left.\varepsilon \nabla 8 \nabla \nabla^{\circ} \varepsilon\right]$

oน8DG $\varepsilon]$

$\left.\varsigma \varepsilon \nabla \varepsilon 9^{\circ} \varepsilon\right]$

$\left.\begin{array}{c}\text { ¿टEग9 } \varepsilon \\ \varepsilon 9699^{\circ} \varepsilon \\ \varepsilon\end{array}\right]$

65899

DOSD6.

$98 \angle 96^{\circ} \varepsilon \longrightarrow$

$\angle 6696^{\circ}$

$\nabla 0129^{\circ}$

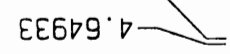

$698 \angle 8 \cdot$

$\angle \nabla \nabla 88^{\circ} \circ$

$6 \angle 268 \cdot \nabla-$

टह906 $\square-$

SELSO $\subseteq-$

LE990 9

Lट9G ' $L$

$060 \angle 2^{\circ} \angle-$

66G $L C^{\circ} L$

OEOIE $\angle 7$

9LटटE $L$

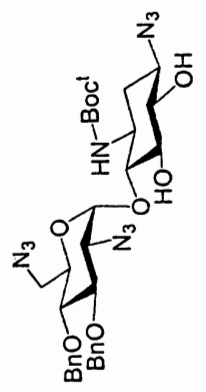

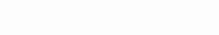

GI IDE ' $L$

ЭEटEE.

BL9EE '

$6 ट 2 \nabla \varepsilon^{\circ} \angle$

GSOSE $\angle$

EIDGE $L$

$9 \angle\left[G E^{\circ} \angle-\right.$

.9998 $\angle-$

I I टट8 $L]$

9७Gट8 $L$ 

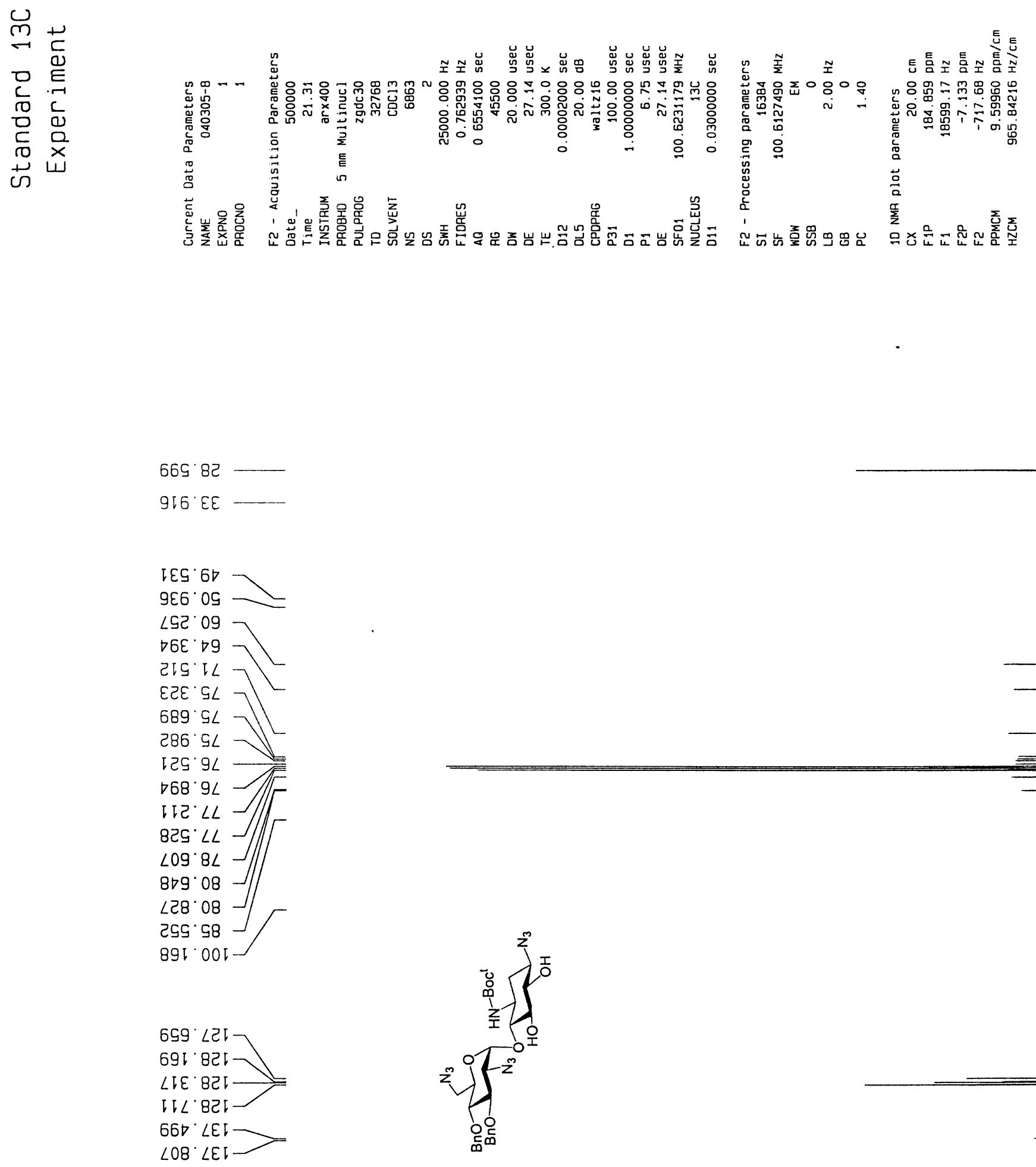

โ๐ 


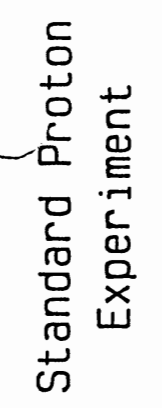
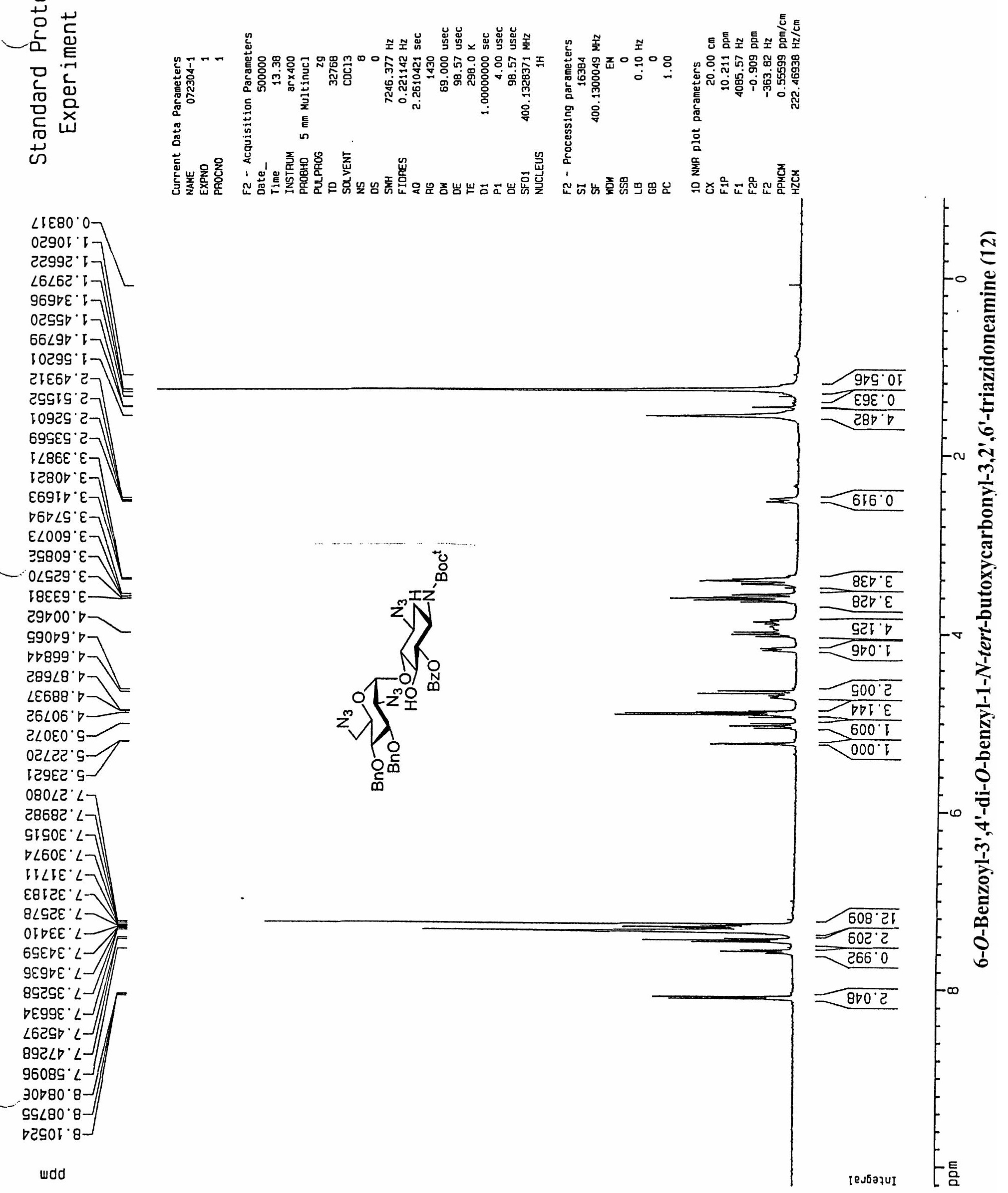

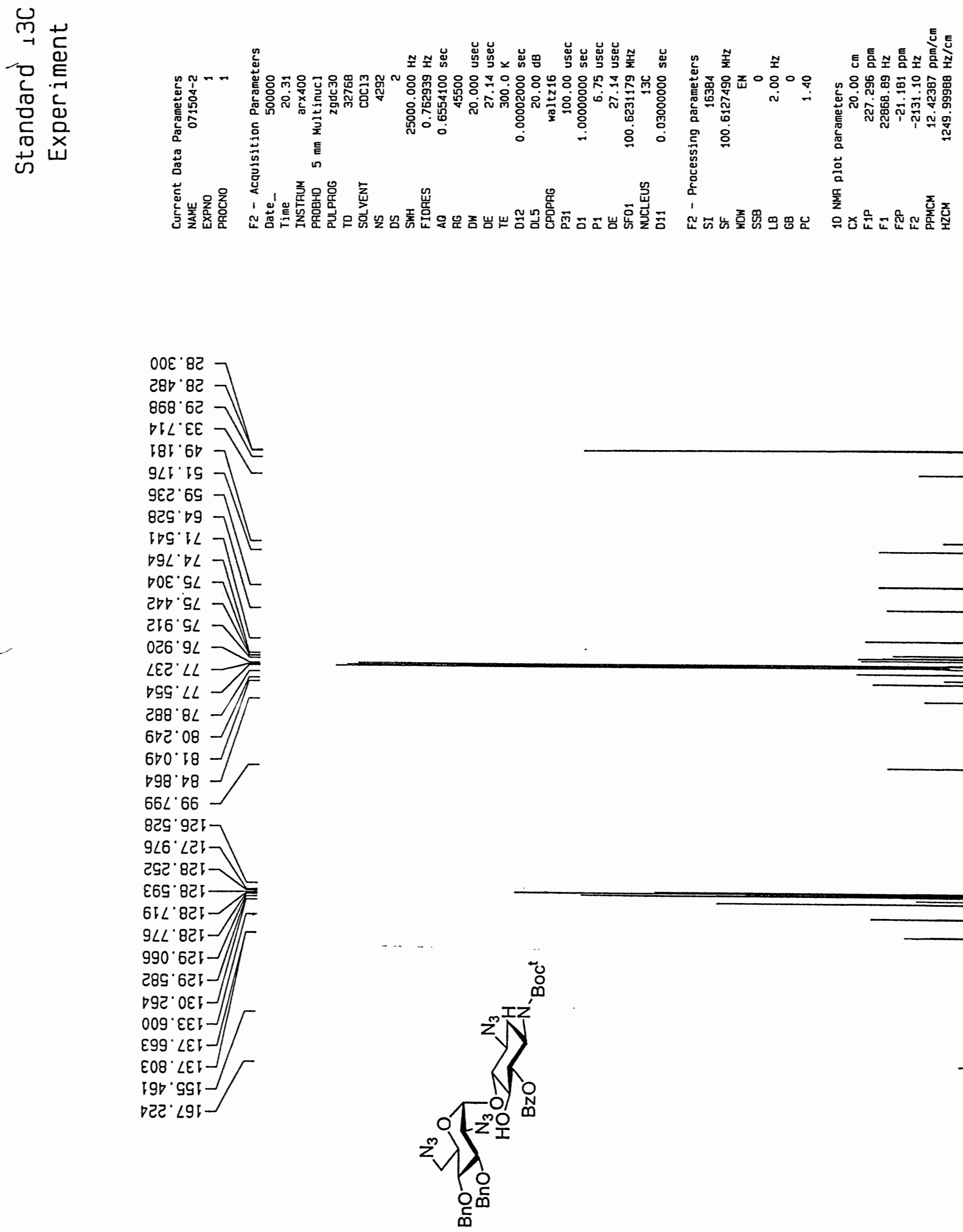


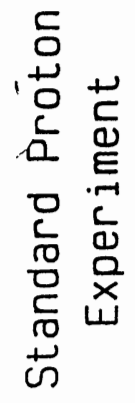
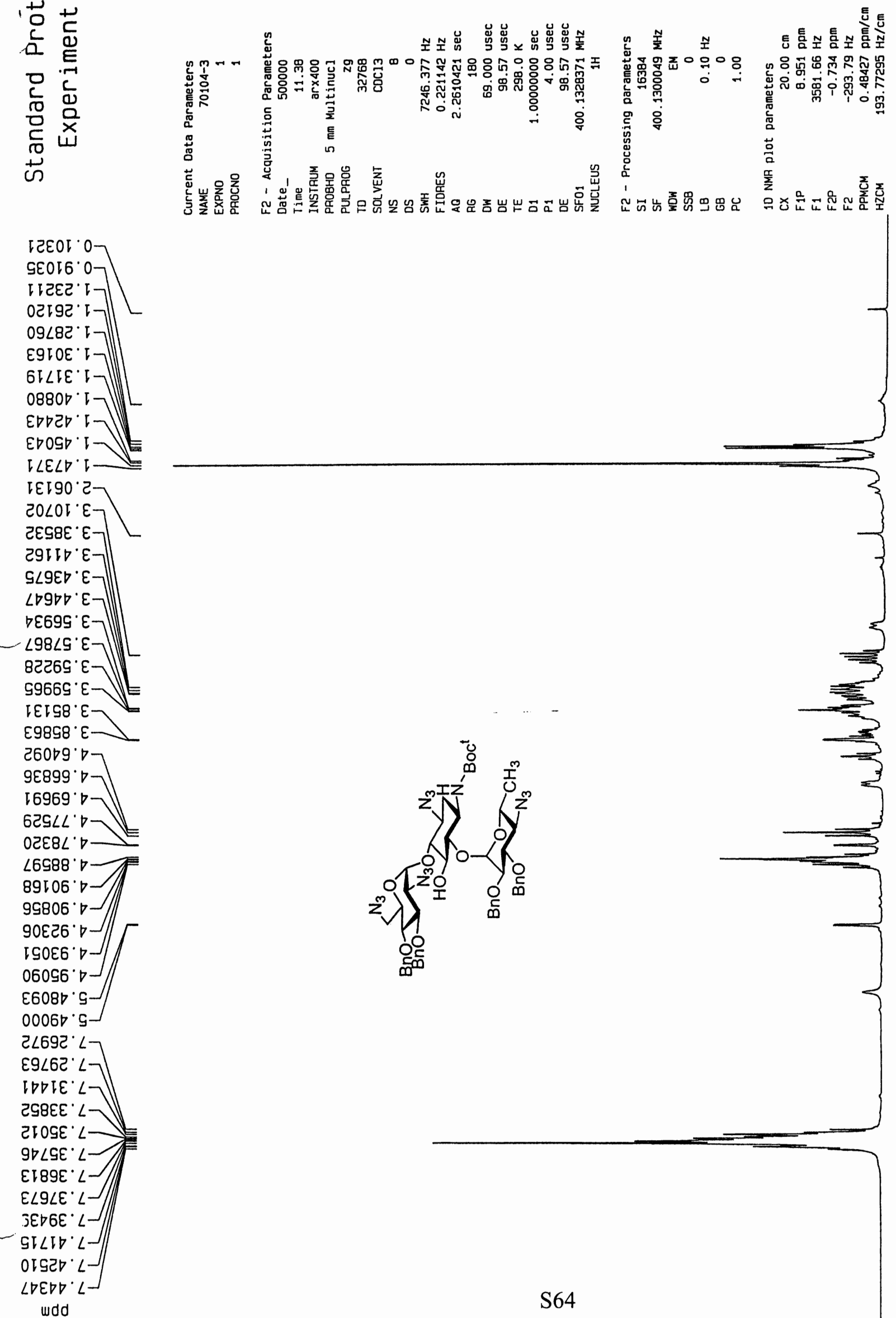


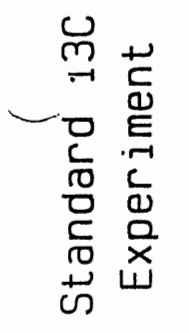
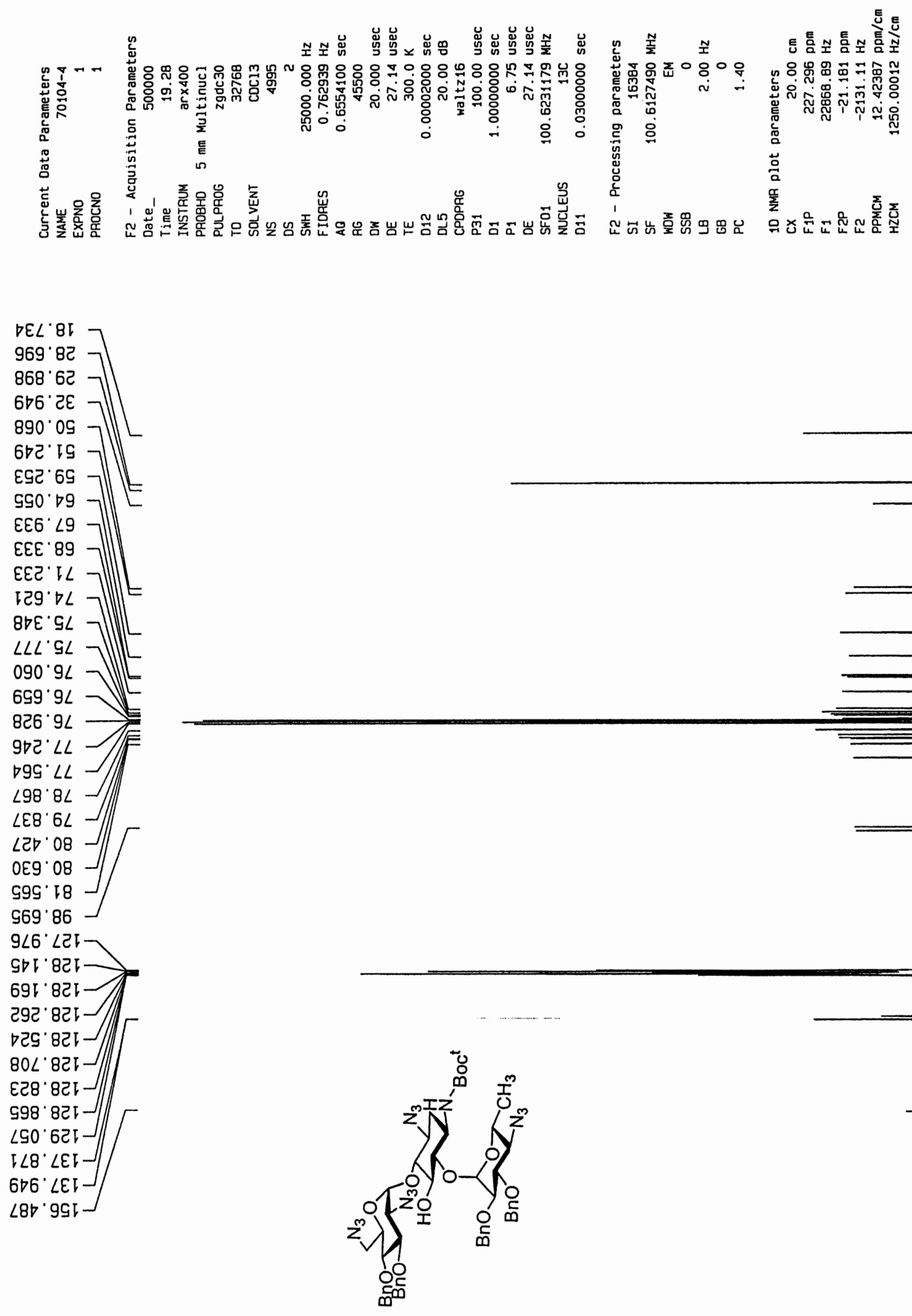


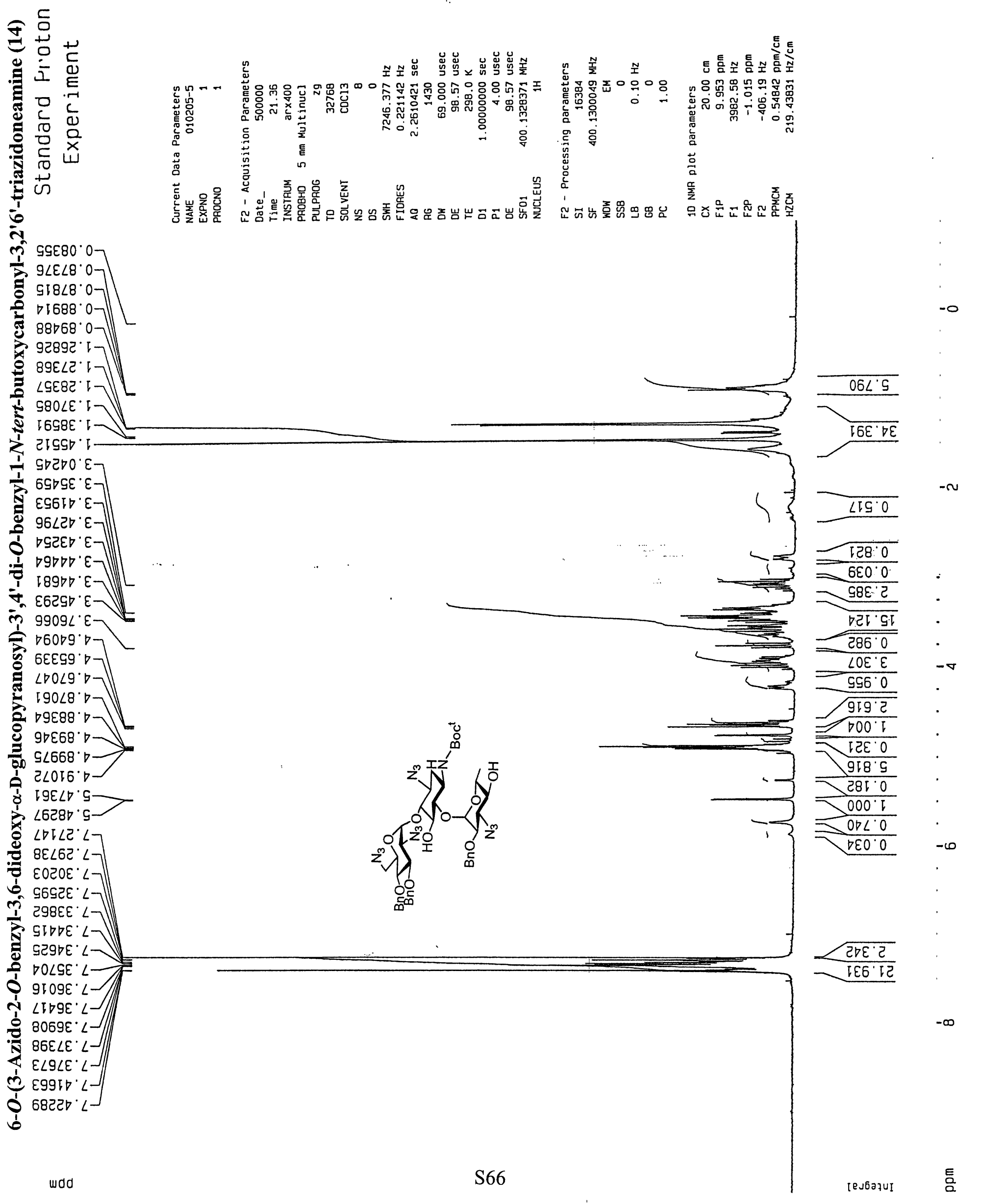




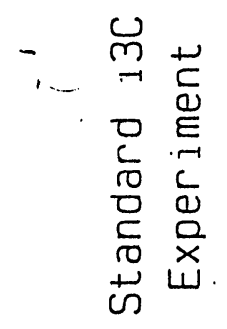

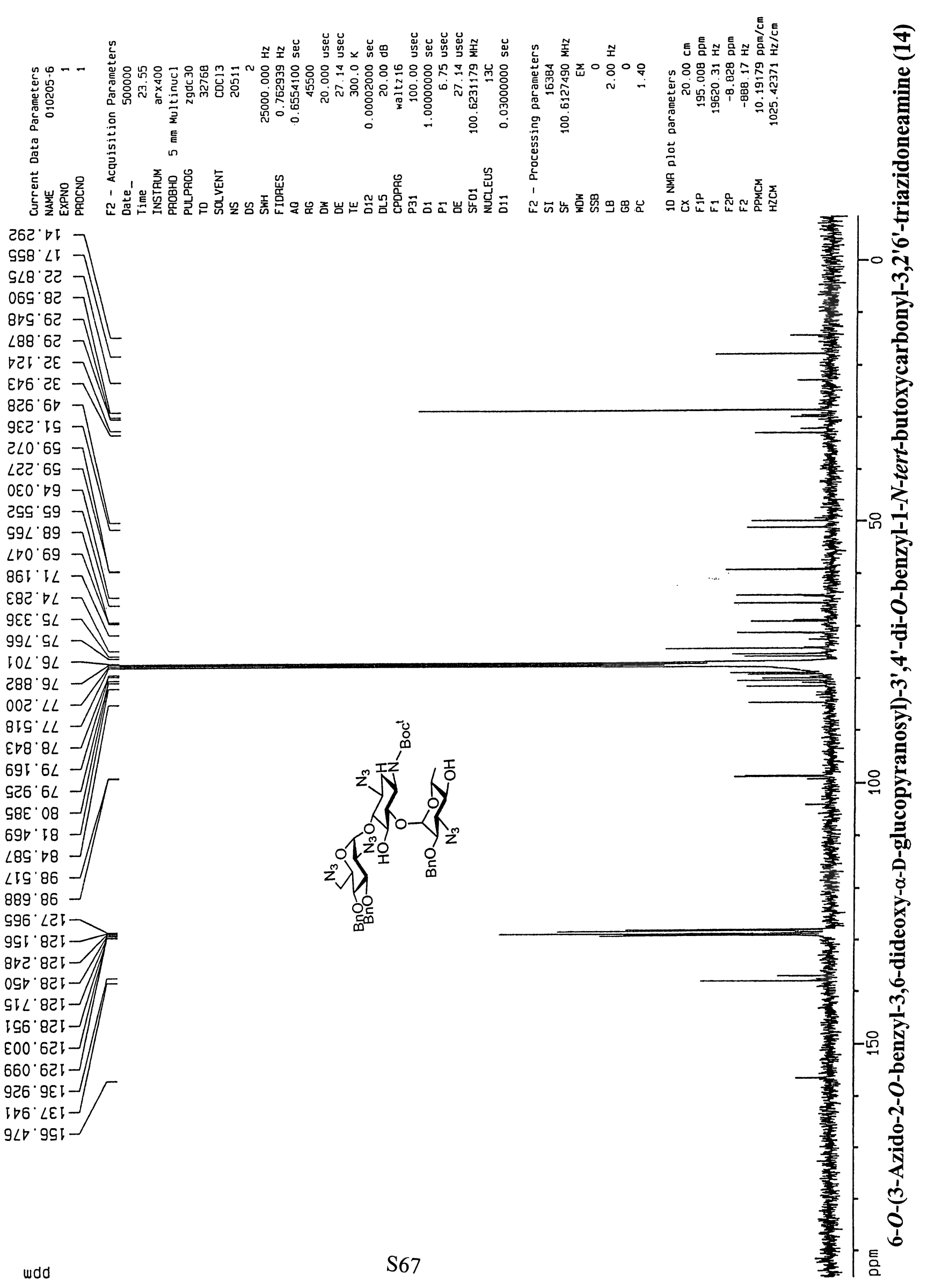




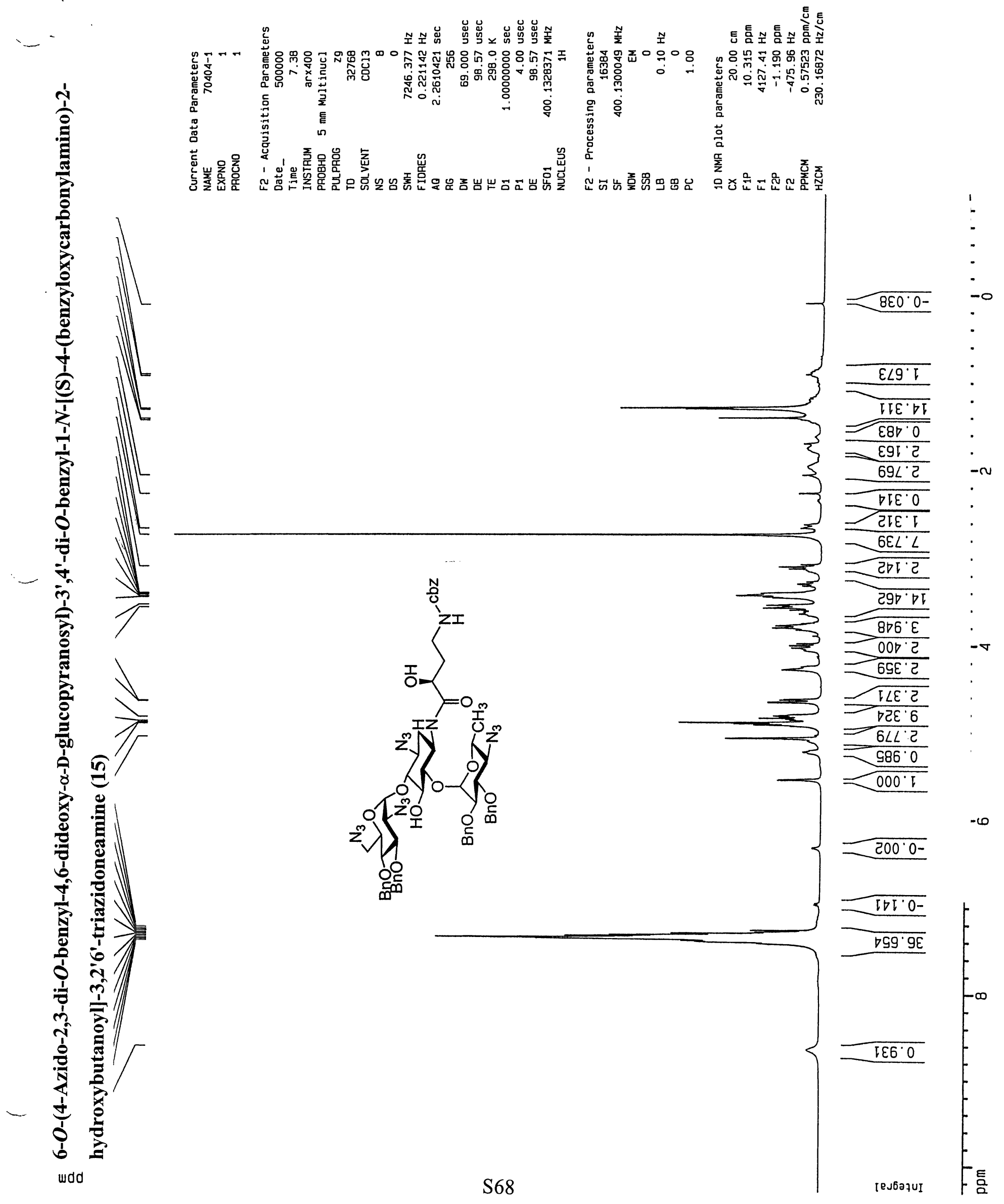



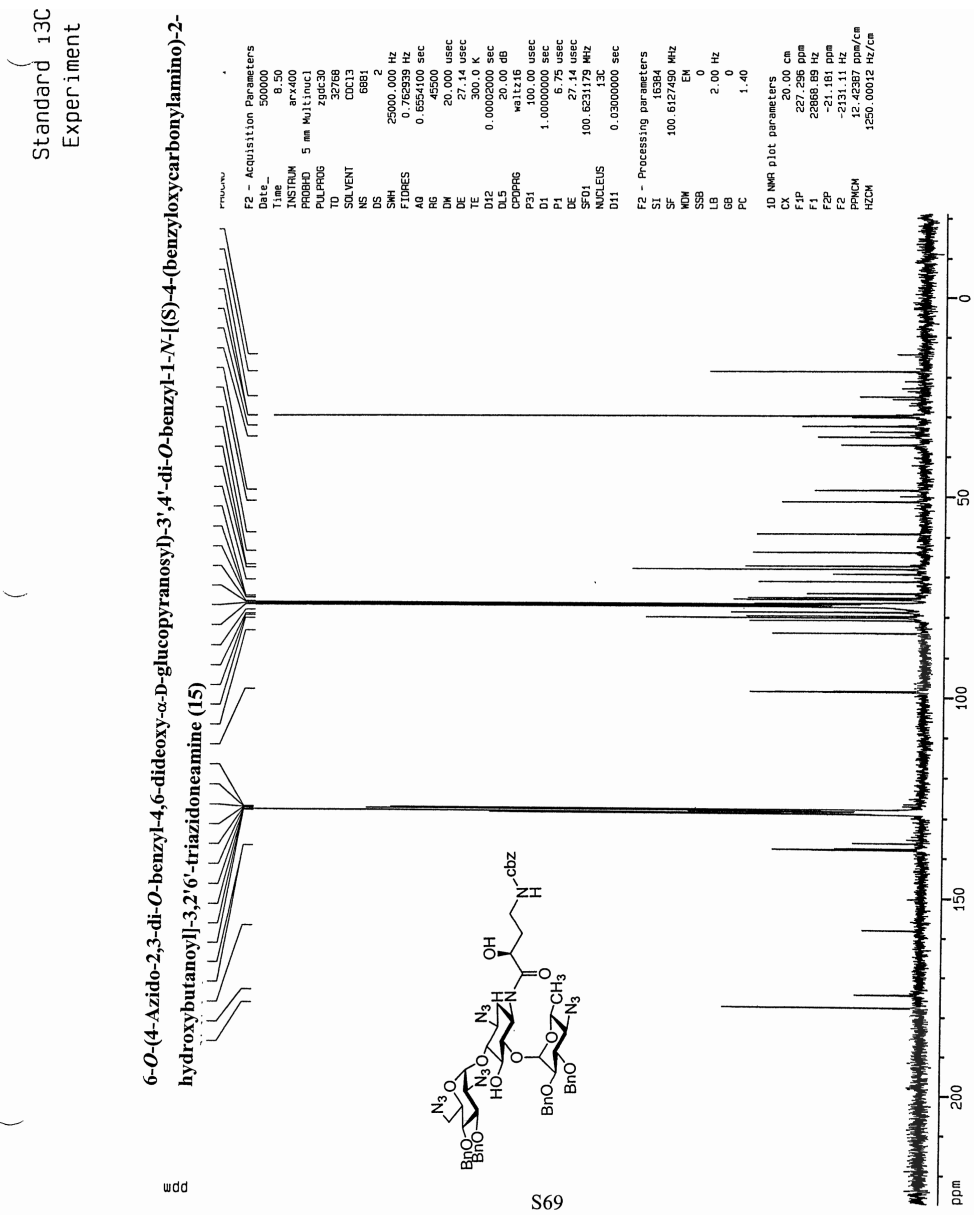


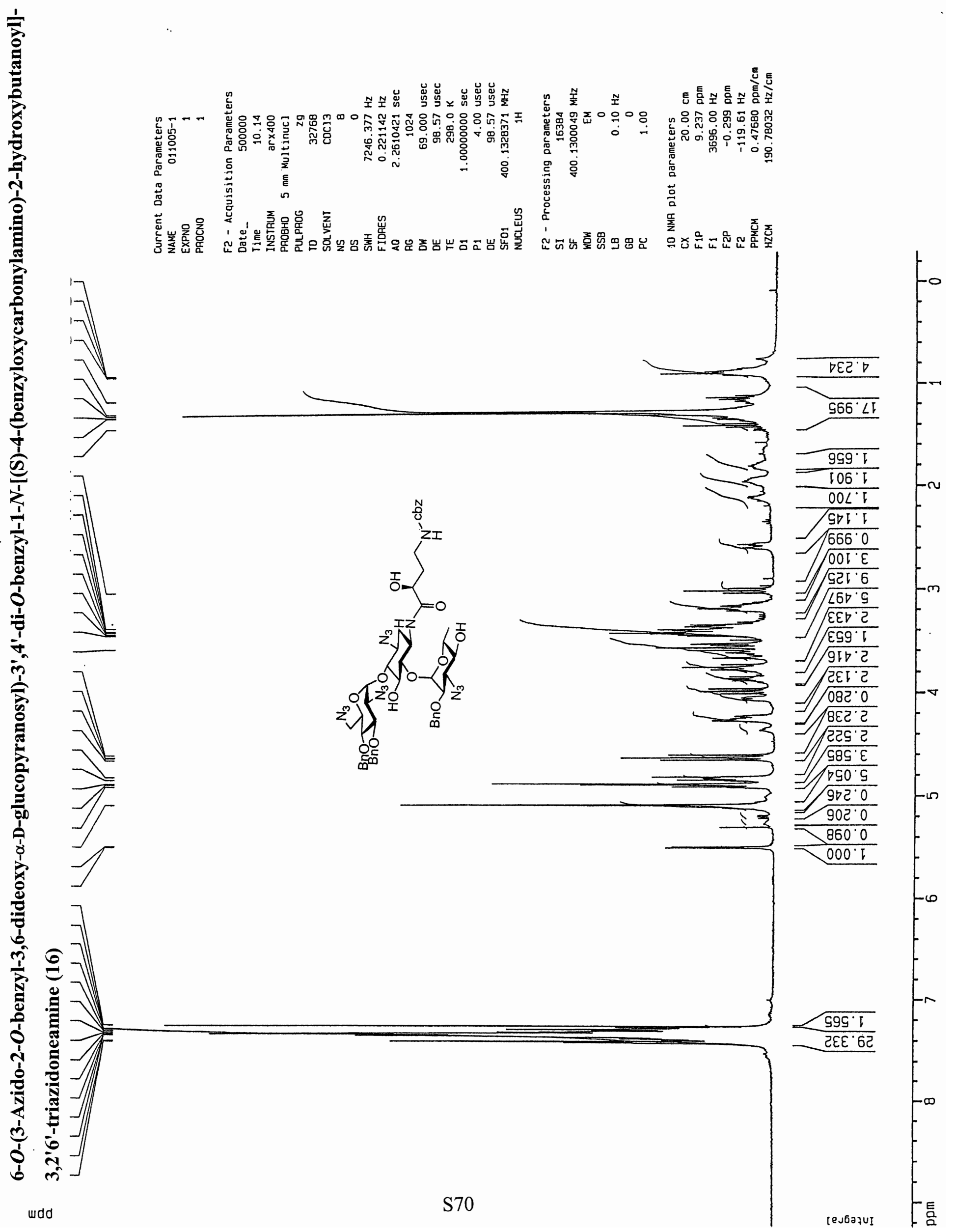




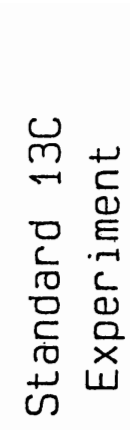

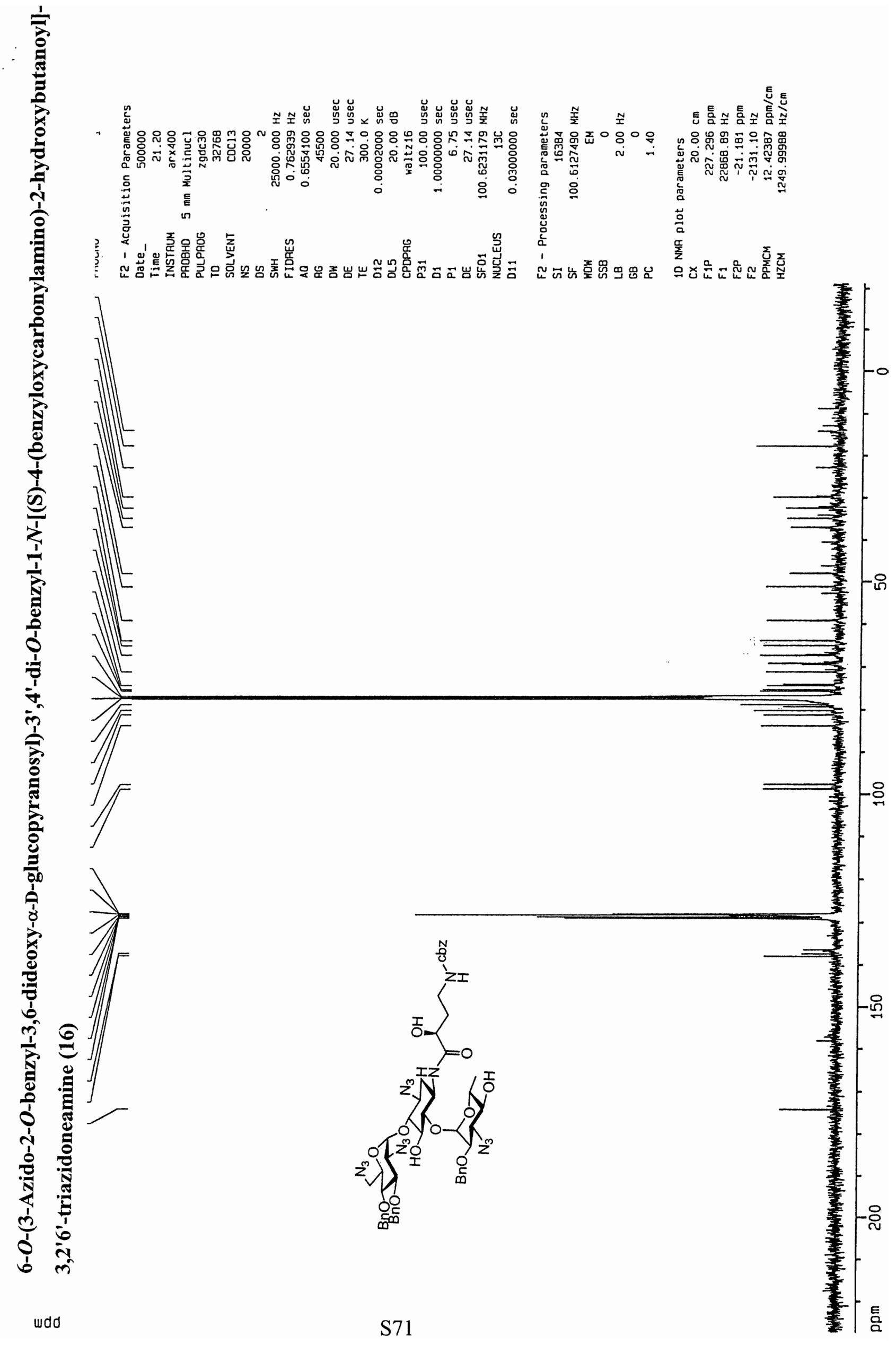




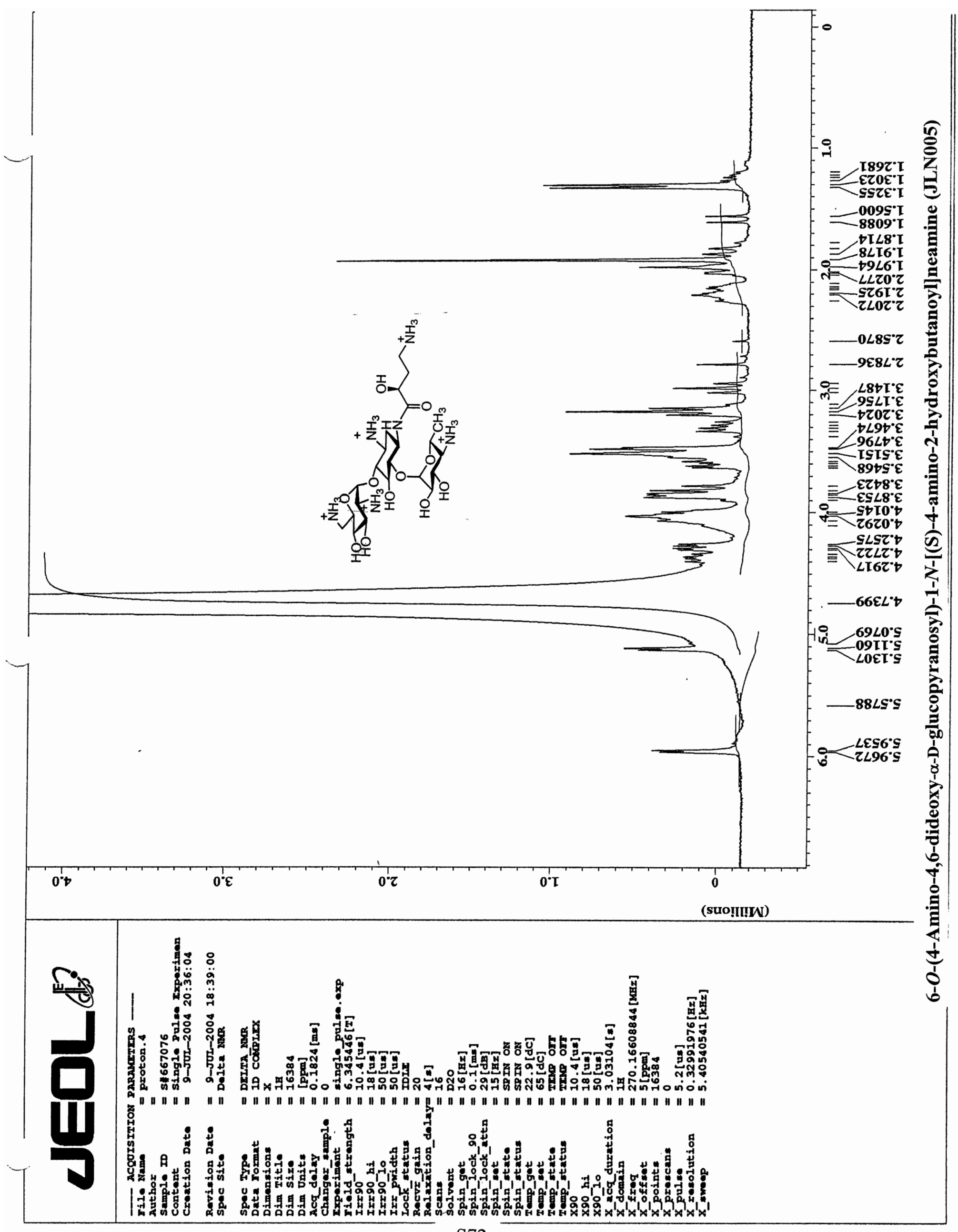




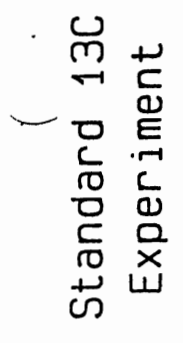
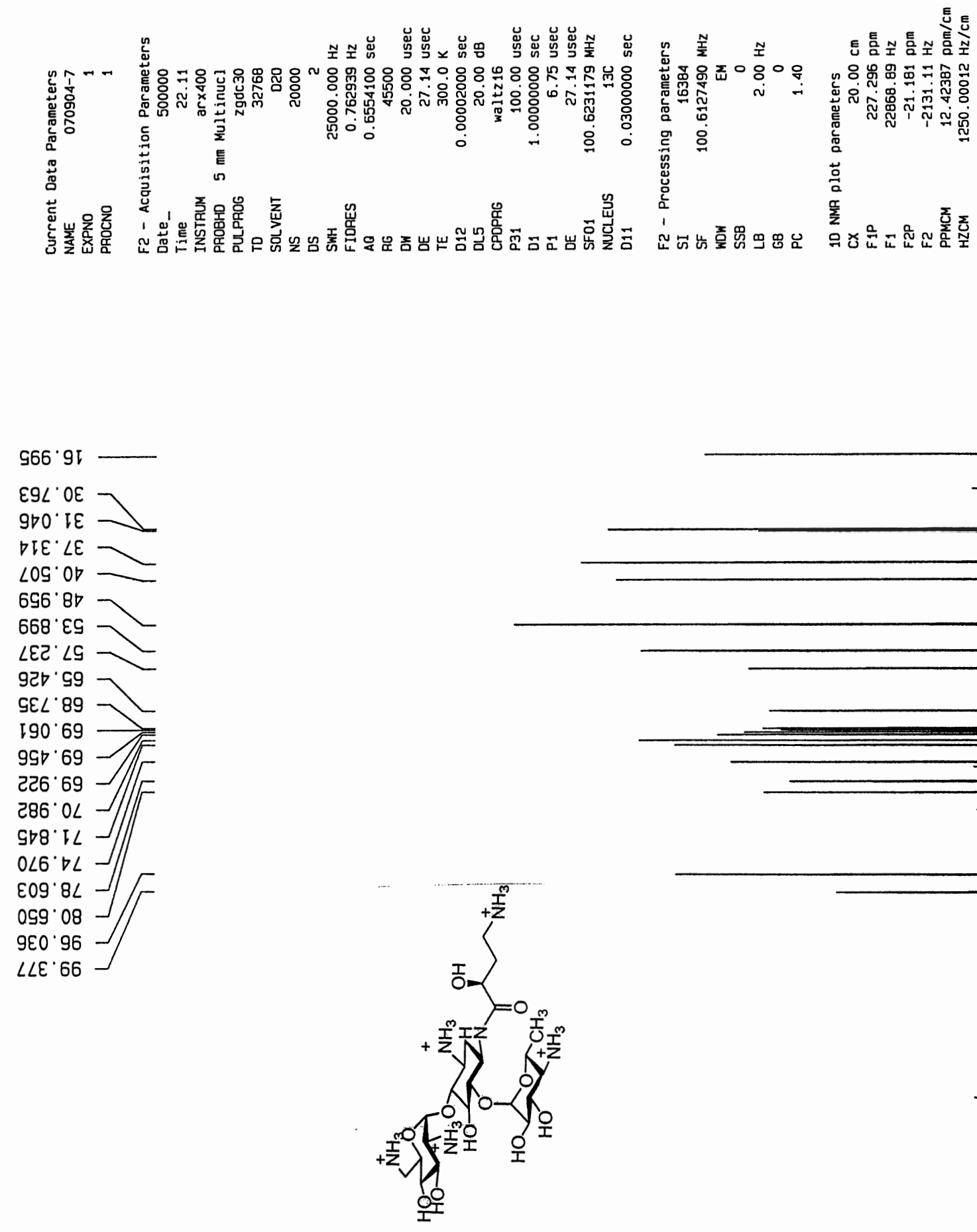

$979^{\circ} G \angle L$ 

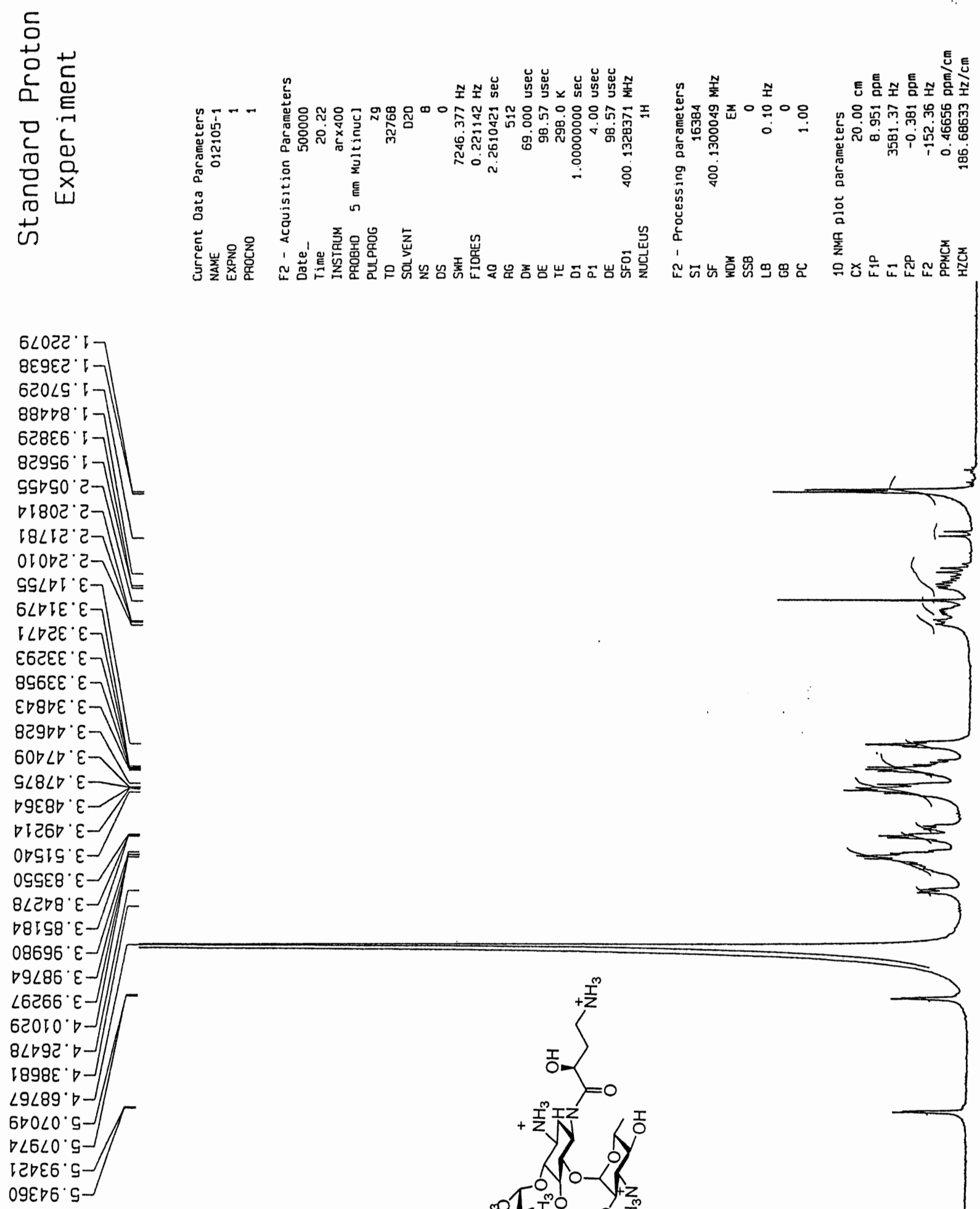

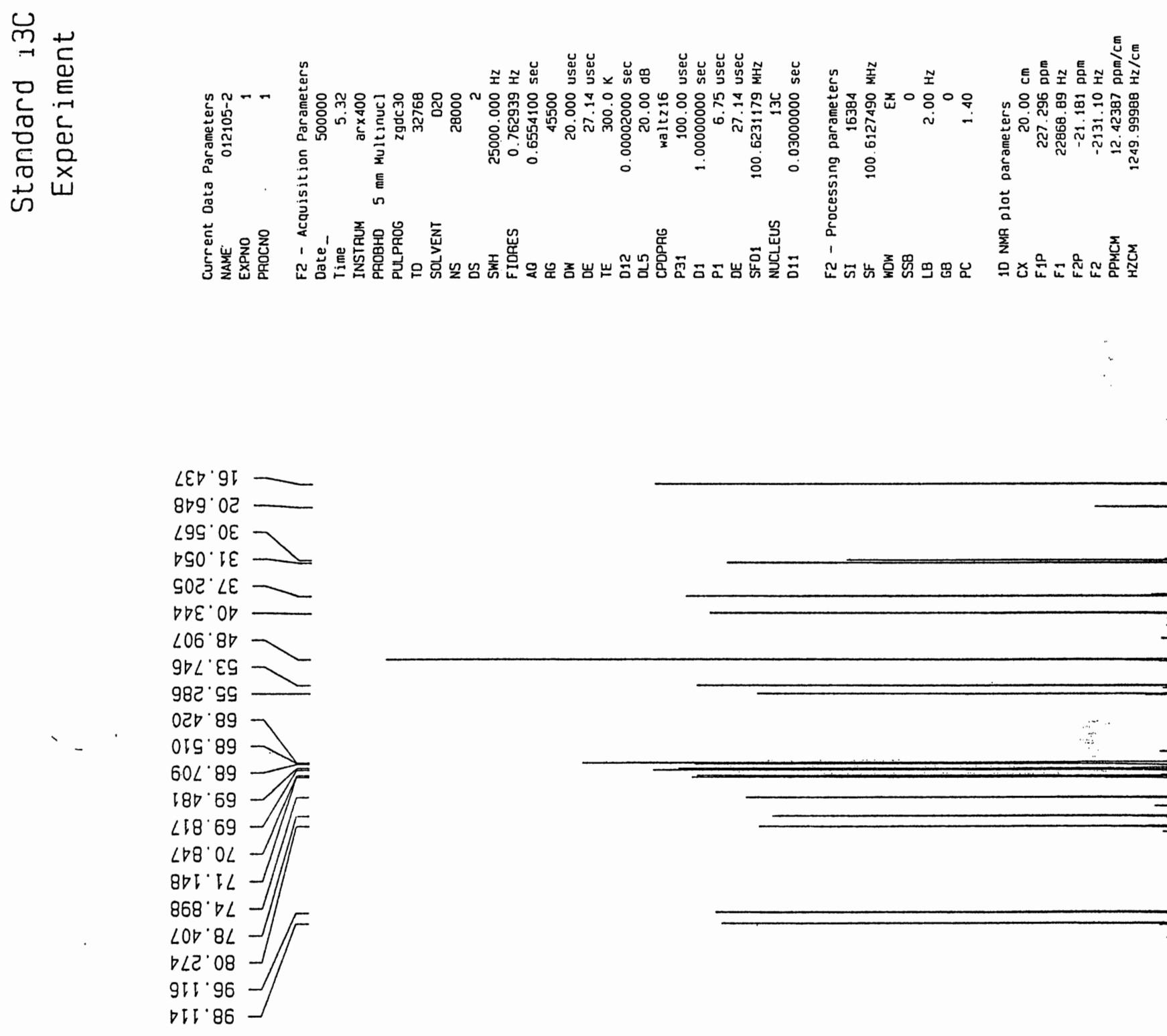

$9 L L^{\circ} G L I-$

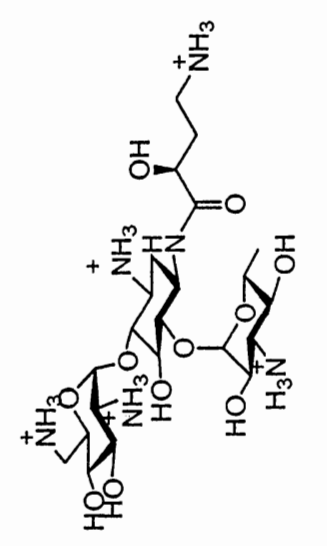



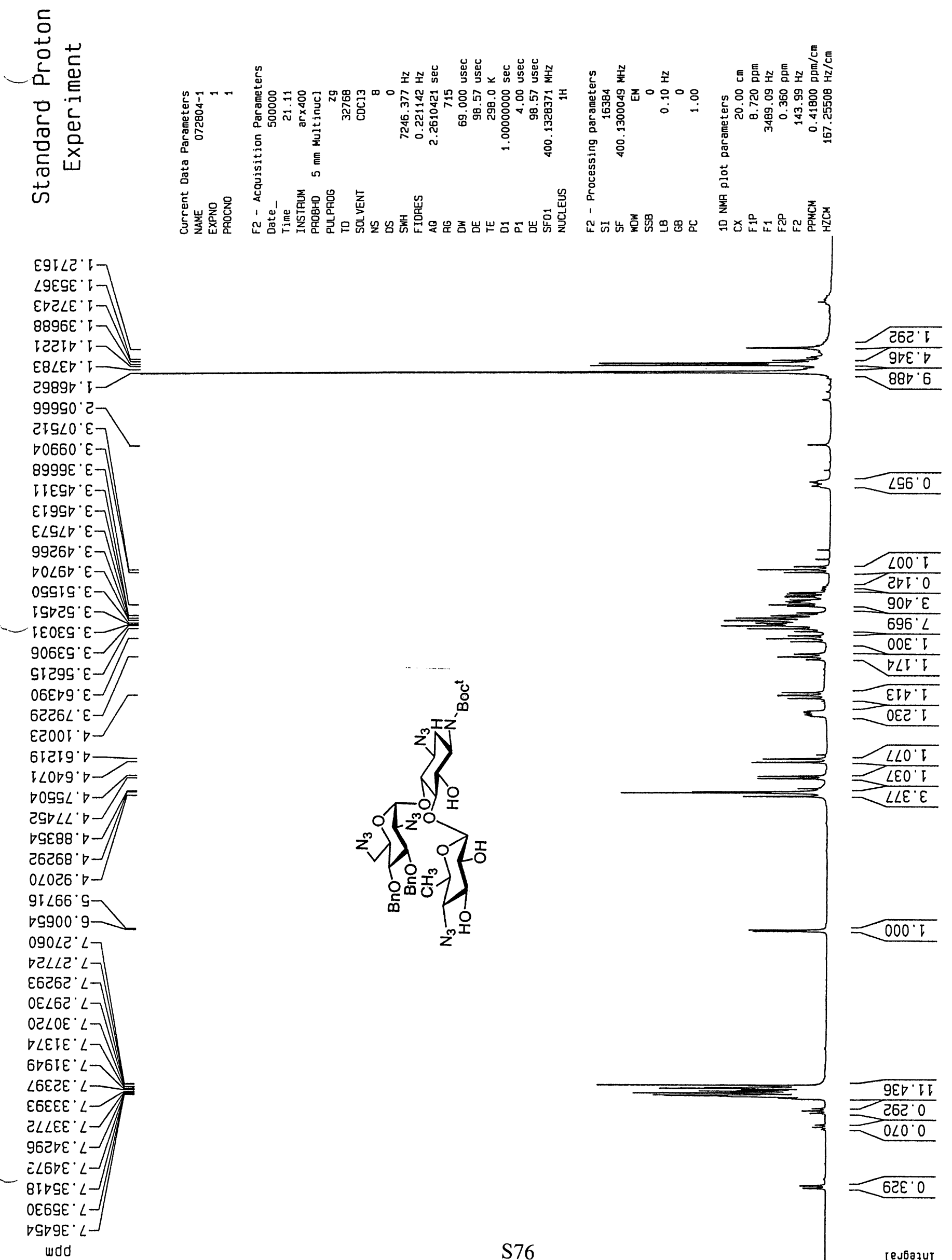

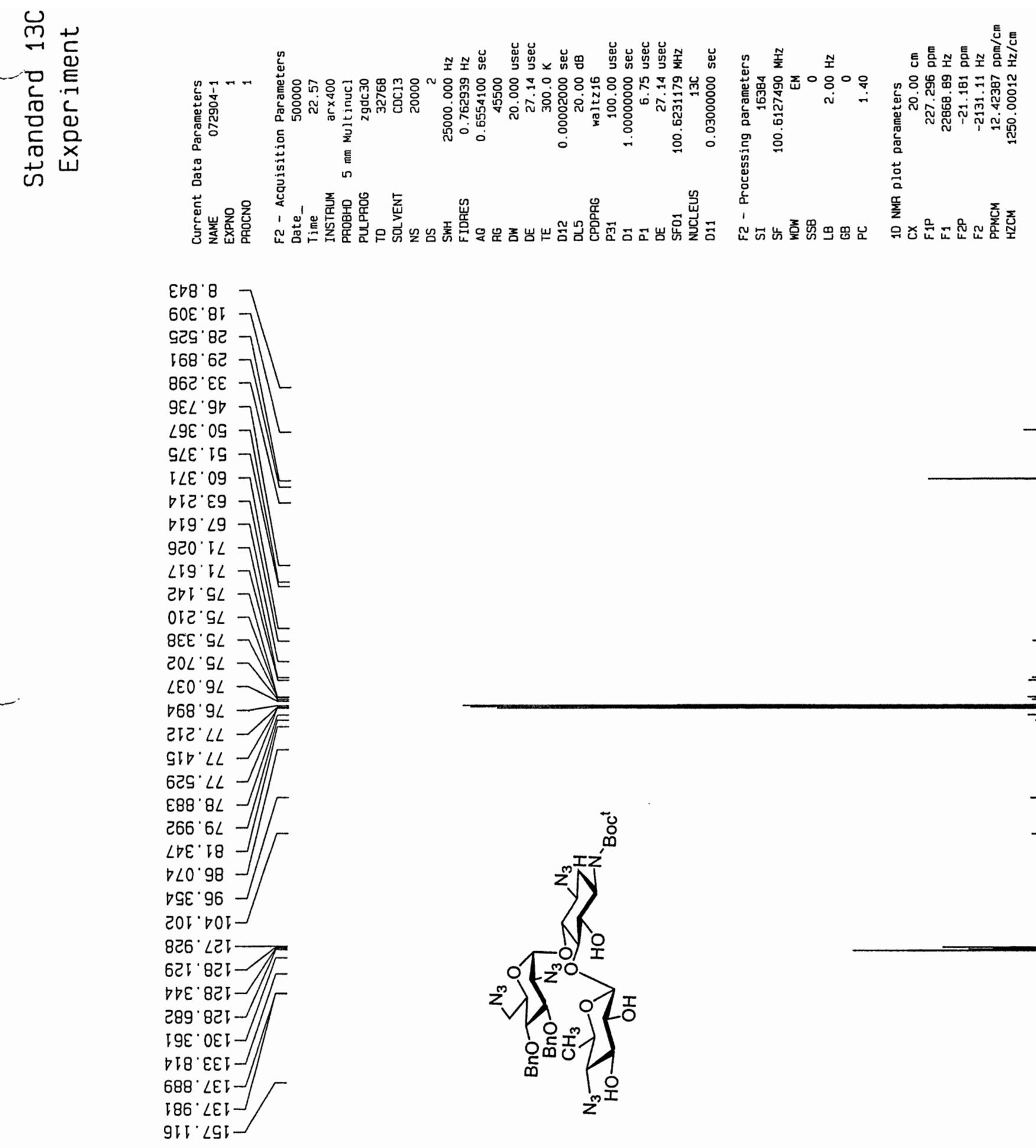


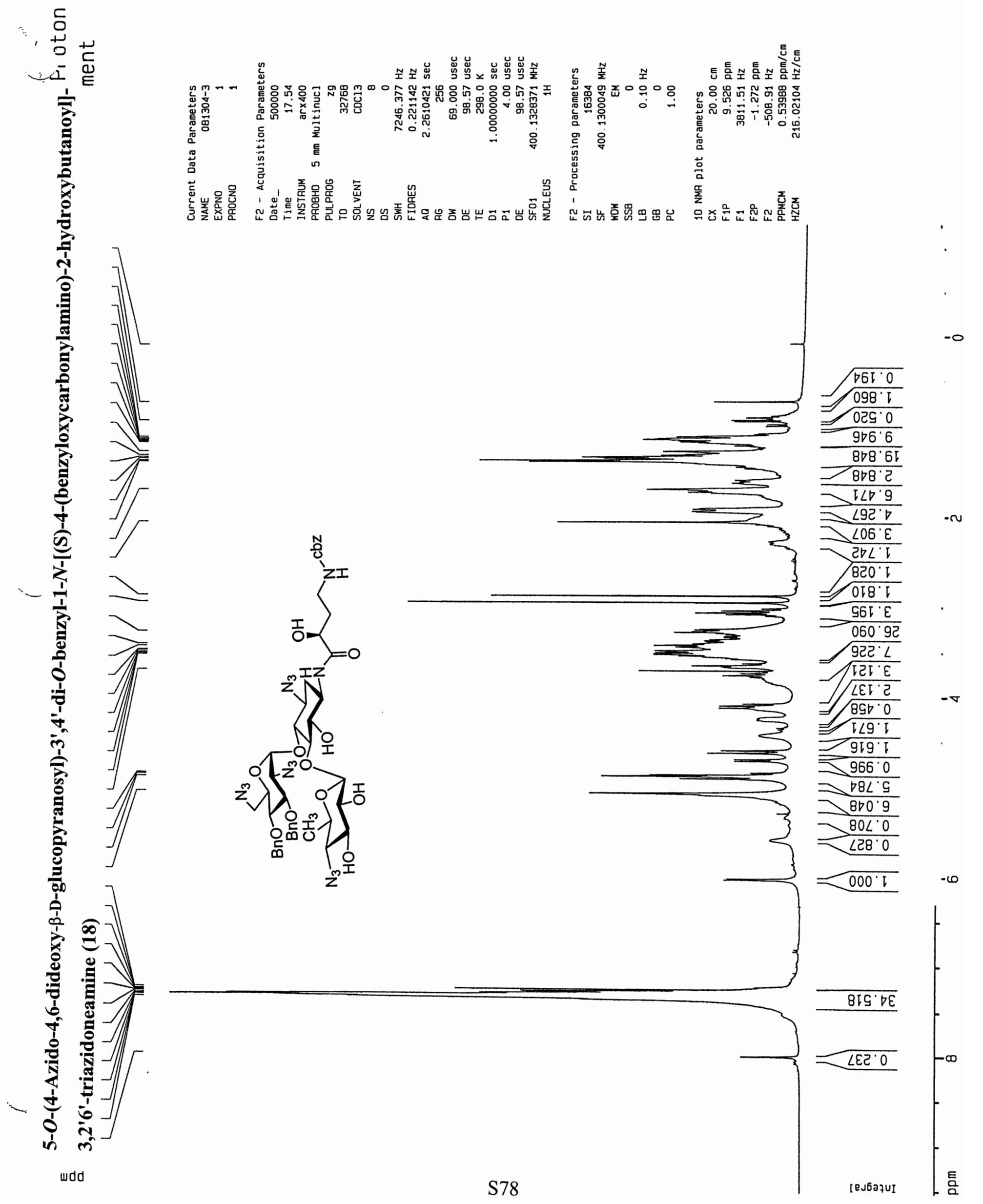




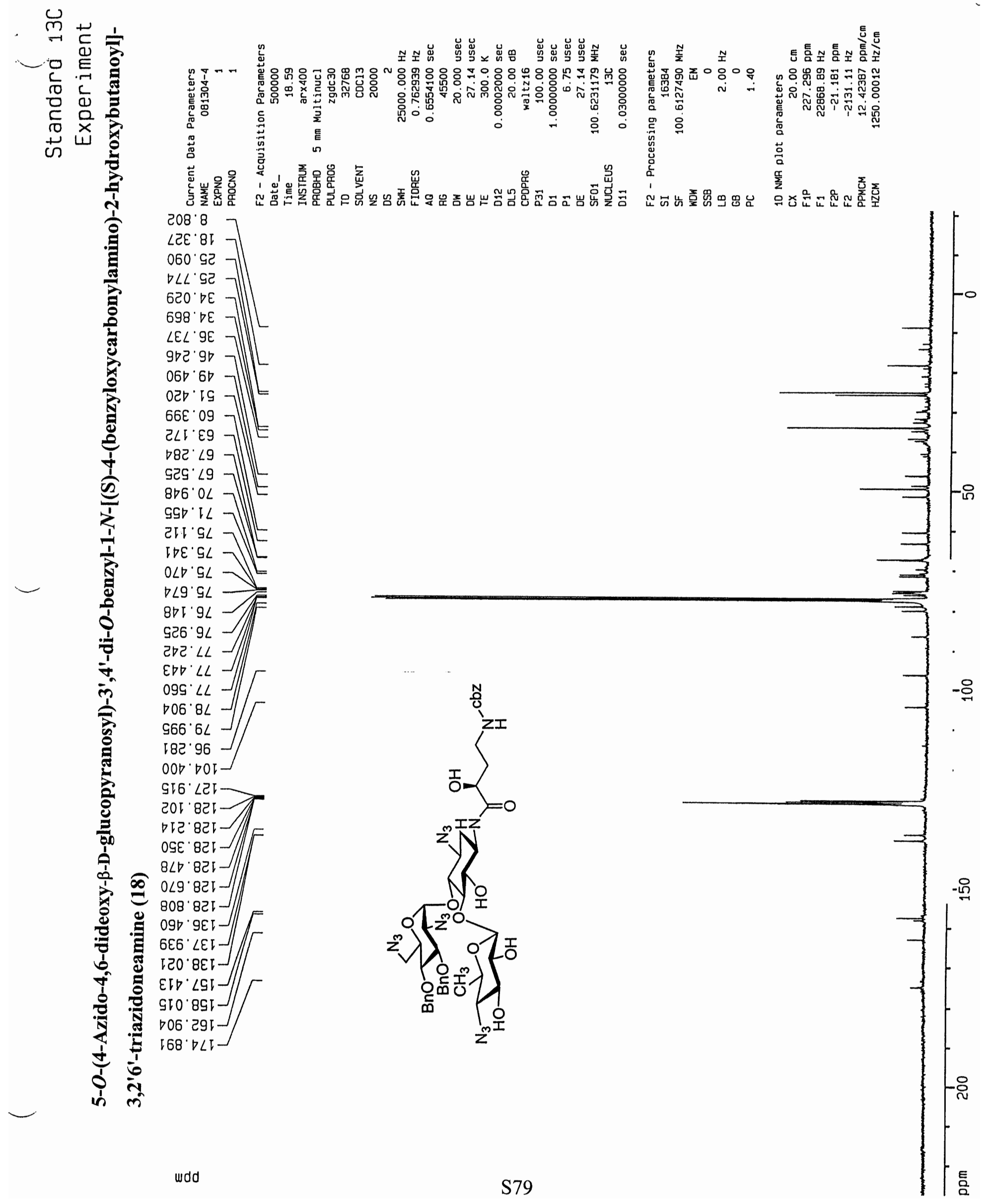



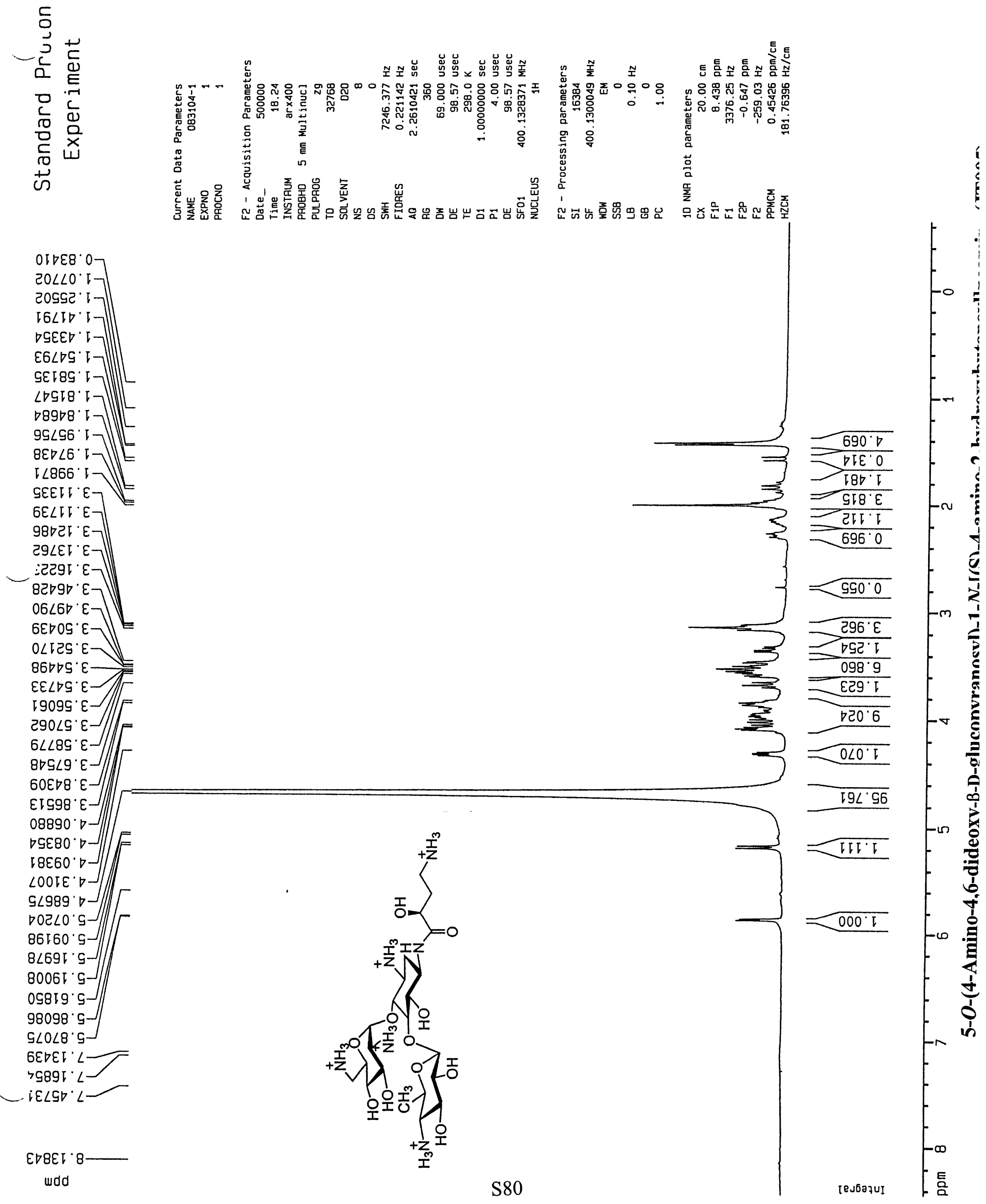


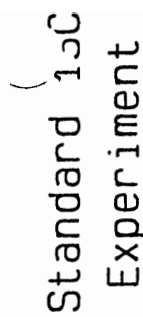

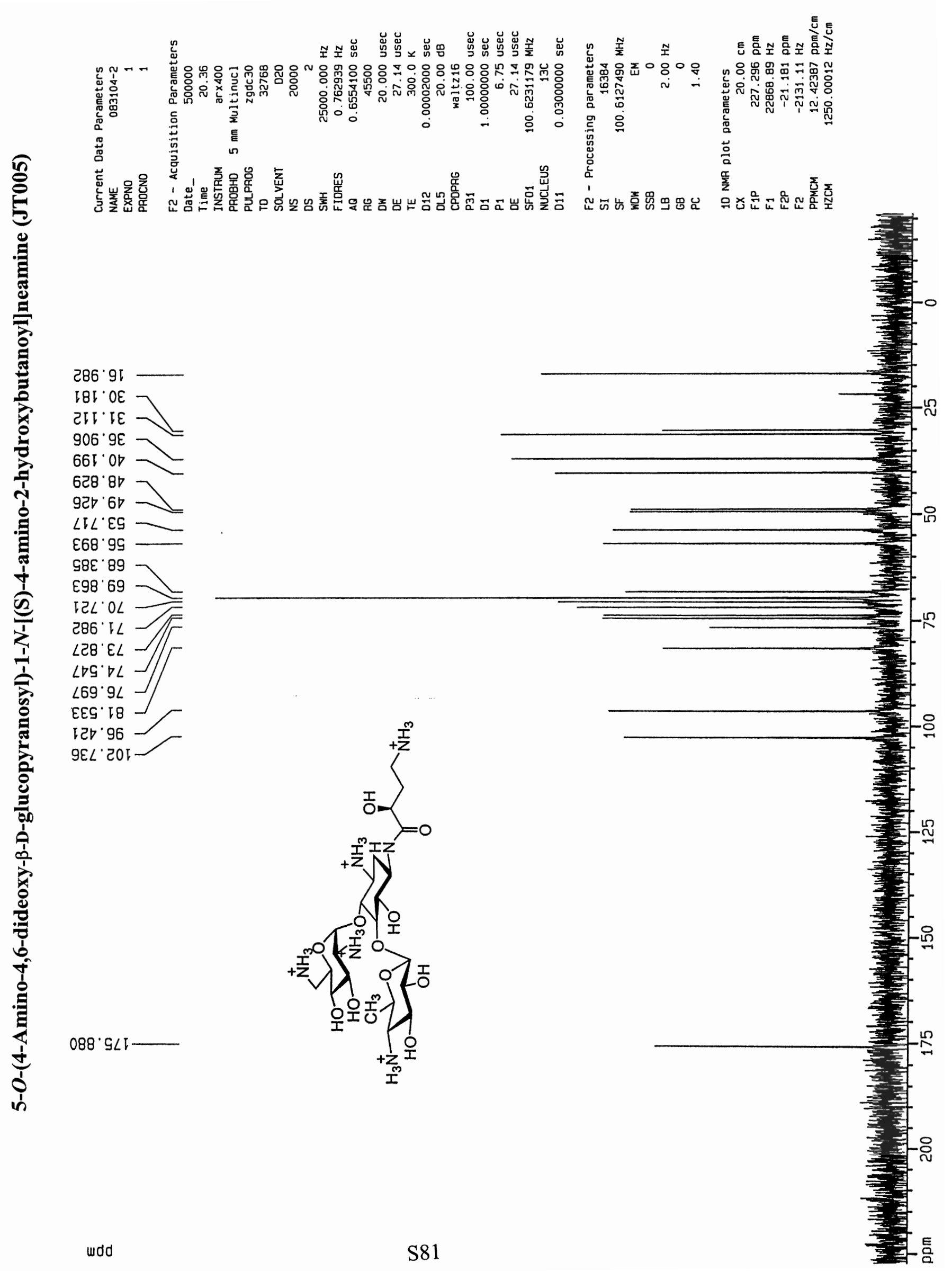

